\title{
Software-defined Mobile Ad Hoc Networks with Trust Management
}

by

\section{Dajun Zhang}

\author{
A thesis submitted to the \\ Faculty of Graduate Studies and Research \\ in partial fulfillment of the requirements for the degree of
}

Master of Applied Science in Electrical and Computer Engineering

Ottawa-Carleton Institute for Electrical and Computer Engineering (OCIECE)

Department of Systems and Computer Engineering

Carleton University

Ottawa, Ontario, Canada, K1S 5B6

August, 2016

(C)Copyright 2016, Dajun Zhang 
The undersigned hereby recommends to the

Faculty of Graduate Studies and Research acceptance of the thesis

\title{
Software-defined Mobile Ad Hoc Networks with Trust Management
}

\author{
submitted by
}

Dajun Zhang, M.A.Sc

in partial fulfillment of the requirements for the degree of

Master of Applied Science in Electrical and Computer Engineering

Prof. F. Richard Yu, SCE, Carleton, Thesis Supervisor

Carleton University

August 2016 


\section{Abstract}

In recent years, mobile ad hoc networks (MANETs) have become popular in different areas. In MANETs, mobile nodes can join or leave the network freely. Because of the mobility of nodes and open wireless medium, MANETs are vulnerable to security attacks. In this thesis, we propose a novel framework of software-defined MANETs with trust management. Specifically, we separate the forwarding plane in MANETs from the control plane, which is responsible for the control functionality, such as routing protocols and trust management in MANETs. Using the on-demand distance vector routing (TAODV) protocol as an example, we present a routing protocol named software-defined trust based ad hoc on-demand distance vector routing (SD-TAODV). Simulation results are presented to show the effectiveness of the proposed softwaredefined MANETs with trust management. 
To my parents and supervisor. 


\section{Acknowledgments}

First of all, I would like to sincerely thank my supervisor, Professor F. Richard Yu for his tremendous time and efforts spent in leading, supporting and encouraging me in the course of my thesis and study. It would not be able for me to finish my thesis without his help and I will always keep to follow his instructions and inspiration to my professional career. I would also thank my colleagues for their understanding and friendship during the time we spent together. Finally, I would like to express my special thanks to my parents for their continuous understanding, supporting and encouraging in my life. 


\section{Table of Contents}

Abstract

Acknowledgments $\quad$ v

Table of Contents vi

$\begin{array}{ll}\text { List of Figures } & \text { ix }\end{array}$

List of Abbreviations $\quad$ xi

1 Introduction $\quad 1$

1.1 Research Overview . . . . . . . . . . . . . . . . . . . 1

1.2 Related Background . . . . . . . . . . . . . . . . . 4

1.2.1 Mobile Ad Hoc Networks . . . . . . . . . . . . . . . . . . . 4

1.2.2 Software-defined Networking . . . . . . . . . . . . . . 6

1.3 Thesis Contributions . . . . . . . . . . . . . . . 7

1.3.1 Accepted and Submitted Papers . . . . . . . . . . . . 8

1.4 Thesis Organization . . . . . . . . . . . . . . 8

2 Overview of MANETs and SDN 10

2.1 Key Technologies of Mobile Ad Hoc Networks . . . . . . . . . . . . . 10

2.1.1 Ad Hoc Theory and its Characteristics . . . . . . . . . . . . 10 
2.1.2 The System Architecture of Mobile Ad Hoc Networks . . . . . 12

2.2 The Categories of MANET Routing Protocols . . . . . . . . . . . . . 13

2.2.1 Proactive Routing Protocols . . . . . . . . . . . . . . 14

2.2.2 On-demand Routing Protocols . . . . . . . . . . . . . . 16

2.2.3 AODV Routing Protocol Introduction . . . . . . . . . 19

2.3 Software Defined Networking and its Interfaces . . . . . . . . . . . . 23

2.4 OpenFlow Key Technology . . . . . . . . . . . . . . 26

2.4.1 OpenFlow Protocol . . . . . . . . . . . . . . . . 27

2.4.2 The Information Interaction of OpenFlow Protocol . . . . . . 27

2.4.3 The Matched Process of Flow Tables . . . . . . . . . . . . . 29

3 MANET Routing Problems and Related Works 31

3.1 The Problems of the MANET Routing Protocols . . . . . . . . . . . . 31

3.2 Related Works . . . . . . . . . . . . . . . . . . . . . 34

4 Trust Based AODV Routing Protocol (TAODV) 37

4.1 Trust Value Calculation Process . . . . . . . . . . . . . . . . 37

4.1.1 Node Trust Calculation Process . . . . . . . . . . . . 38

4.1.2 Path Trust Calculation Process . . . . . . . . . . . . . 39

4.1.3 The Objective Function of TAODV . . . . . . . . . . . 40

4.2 Route Discover Process of TAODV . . . . . . . . . . . . . . 41

5 Software-defined Mobile Ad-hoc Networks based on TAODV 44

5.1 Framework Description . . . . . . . . . . . . . . . . . . 44

5.2 The Control and Forwarding Logic Separation Method . . . . . . . . 48

5.3 The Design Method of Controller . . . . . . . . . . . . . . . . 49

5.3.1 The Working Process of the Controller . . . . . . . . . . 50

5.3.2 The Implementation of OpenFlow Protocol in SD-TAODV . . 51 
5.4 The Design of Forwarding Nodes . . . . . . . . . . . . . . . 52

6 Simulation Results and Discussions $\quad 54$

6.1 SD-TAODV Simulation Setup . . . . . . . . . . . . . 54

6.2 Evaluation . . . . . . . . . . . . . . . . 56

7 Conclusions and Future Works $\quad 64$

$\begin{array}{ll}\text { List of References } & 67\end{array}$

$\begin{array}{ll}\text { Appendix A Simulation Programs } & 74\end{array}$ 


\section{List of Figures}

2.1 The MANET routing protocols $[1] . \ldots \ldots \ldots$

2.2 The frame of the RREQ packet. . . . . . . . . . . . . 20

2.3 The frame of the RREP packet. . . . . . . . . . . . . . . . . 21

2.4 A route reply process. . . . . . . . . . . . . . . . . 22

2.5 Route discovery process. . . . . . . . . . . . . . . . . . . . 23

2.6 The SDN architecture. . . . . . . . . . . . . . . . 25

2.7 The OpenFlow switch and controller interaction using OpenFlow protocol. . . . . . . . . . . . . . . . . . 26

2.8 The controller-to-switch message. . . . . . . . . . . . . . . . 28

2.9 The asynchronous message. . . . . . . . . . . . . . . . . . . 28

2.10 The symmetric message. . . . . . . . . . . . . . . . . . . . . 29

2.11 The matched process of OpenFlow switch. . . . . . . . . . . 30

4.1 An example of the TAODV calculation process. . . . . . . . . . . . 42

5.1 Traditional OpenFlow switches and controller interaction using OpenFlow messages. . . . . . . . . . . . . . . . . . . . . . . . . 45

5.2 An example Mobile ad-hoc network using SD-TAODV mechanism. . . 46

5.3 The working process of switches based on TAODV protocol. . . . . . 47

5.4 The working process of control node based on TAODV protocol. . . . 50

5.5 The working process of forwarding node based on TAODV protocol. . 52 
6.1 The average ETE delay of SD-TAODV with trust value in different data rates. . . . . . . . . . . . . . . . . 57

6.2 The average ETE delay of SD-TAODV with trust value and hop count in different data rates. . . . . . . . . . . . . . . . . 57

6.3 The average ETE delay comparison of SD-TAODV with the time variation in 5.5 Mbps. . . . . . . . . . . . . . . . . 58

6.4 The average ETE delay comparison of SD-TAODV with the time variation in 11 Mbps. . . . . . . . . . . . . . . . . . . . 59

6.5 The average throughput comparison of SD-TAODV with different data rates. . . . . . . . . . . . . . . . . . 59

6.6 The average throughput comparison of SD-TAODV with time variation in 5.5 Mbps. . . . . . . . . . . . . . . . . 60

6.7 The average throughput comparison of SD-TAODV with time variation in 11 Mbps. . . . . . . . . . . . . . . . . . 61

6.8 The average throughput comparison of SD-TAODV number of nodes. 61

6.9 Total message overhead comparison in different number of nodes. . . 61

6.10 PDR versus the number of nodes. . . . . . . . . . . . . . . . 62

6.11 PDR versus the node velocity. . . . . . . . . . . . . . . . . 62 


\section{List of Abbreviations}

$\begin{array}{ll}\text { AODV } & \text { Ad Hoc On-demand Distance Vector Routing } \\ \text { CLI } & \text { Command Line Interface } \\ \text { DSDV } & \text { Destination sequenced distance vector } \\ \text { DSR } & \text { Dynamic Source Routing } \\ \text { DARPA } & \text { Defense Advanced Research Projects Agency } \\ \text { LLC } & \text { Logic Link Control } \\ \text { MAC } & \text { Media Access Control } \\ \text { MANETs } & \text { Mobile Ad Hoc Networks } \\ \text { NOS } & \text { Network Operating System } \\ \text { OLSR } & \text { Optimized Link State Routing }\end{array}$


QoS Quality of Service

RREP Route Reply

RREQ Route Request

RRER Request Error

SDN Software-defined Networking

SD- $\quad$ Software-defined Trust based On-demand Distance Vector Routing

TAODV

T-RREP Trust Route Reply

T-RREQ Trust Route Request

WRP Wireless Routing Protocol 


\section{Chapter 1}

\section{Introduction}

\subsection{Research Overview}

With the rapid development of network technologies, network scales continue to expand. Meanwhile, as the rapid innovation of Internet services, computer networks have been widely used in various fields, such as military, science, education, and business. People can communicate with each other and can get information from websites through the computer networks. Computer networks profoundly impact people's learning, work and life. However, since the emerging network technologies such as Internet of Things and software-defined networking have been adopted in different areas, the computer networks gradually come out many limitations [2].

In the TCP / IP architecture, network devices (such as switches, routers, etc.) are generally composed of control planes and forwarding planes [3]. The control planes are responsible for forwarding strategies based on gateway protocols and routing protocols. Forwarding planes comprise the entities to execute control logic from the control planes. The main responsibilities of the forwarding plane include data encapsulation, package de-encapsulation, and packet transmission [4]. However, the control planes and forwarding planes in traditional networks are all concentrated 
on the network devices. This characteristic makes users difficult to personalize the network according to the various requirements. The tight coupling of the control plane and the forwarding plane result in a series of problems, especially the users hard to manage and adjust the network. [5].

In order to solve these problems, software-defined networking (SDN) has been first introduced by the Clean Slate research group at Stanford University [6]. Software defined networking is an emerging network architecture where network control is decoupled from forwarding plane, and it is directly programmable [7]. Due to the separation of the control planes and the forwarding planes, network nodes only act as forwarding devices and network control logic moves into a logic centralized controller or a networking operating system [8]. On the other hand, depending on the network scale, the control plane may have more than one controllers. If multiple controllers are deployed, a high-speed and reliable distributed network control mechanism can be formed in the peer-to-peer manner. In a word, no matter with either centralized or distributed organizing schemes of the controller, network nodes in the forwarding plane simply forward data through checking flow table(s) that are managed by the controller(s) in the control plane. SDN provides a cost-effective networking approach that aims to reduce the cost of wireless networks and improves the network performance.

On the other hand, with the miniaturization of mobile end devices, mobile ad hoc networks (MANETs) [9] become popular in wide range of fields. For example, they can be used in military, catastrophes, expeditions and so on. Meanwhile, researchers propose higher requirements for MANETs in recent years.

MANETs are evolved from US defense advanced research projects agency (DARPA) [10], which was a wireless network technology branch in the US Department of Defense in the 1970s. DARPA was mainly used in the military field [11]. The 
high mobility feature of MANETs enables its wide applications, and thus the research on MANETs attracts much attention from researchers at the United States California Polytechnic State University, Los Angeles adaptive wireless mobile lab, "University of Maryland" Mobile Computing, and multimedia laboratory, etc. [12].

The self-organizing feature of MANETs indicates that no inherent communication network infrastructure is required. MANETs can quickly form temporary networks and establish network communications. In the case that the network infrastructures are seriously damaged by disasters, such as floods, earthquakes, and typhoons, MANETs become the proper method to establish network communications.

However, the distributed network topology, the network node mobility and the wireless channel variation result in performance fluctuation of MANETs, and thus the network control becomes a key challenge. In addition, various node hardware and software configuration may introduce further network control and management complexity.

MANET routing protocols are generally either table-driven routing protocols or on-demand routing protocols [13]. Destination sequenced distance vector (DSDV) [14] is one of the table-driven protocols, and ad hoc on-demand distance vector routing (AODV) [15] is a typical on-demand routing protocol of MANETs. The main objective of those protocols is to find the shortest data forwarding paths from source to destination.

We can use similar techniques as in SDN to solve MANET problems. In this thesis, with the recent advances in SDN, we propose a novel framework of software-defined MANETs with trust management. Specifically, we separate the forwarding plane in MANETs from the control plane, which is responsible for the control functionalities, such as routing protocols and trust management in MANETs. As AODV [16] protocol is frequently used in MANETs, we utilize AODV as an example to implement our 
proposed SDN-base framework in MANETs. In addition, we move the AODV control logic and the trust management into the control node. Simulation results demonstrate that our software-defined trust based ad hoc on-demand distance vector routing (SDTAODV) can improve the network performance significantly.

\subsection{Related Background}

\subsubsection{Mobile Ad Hoc Networks}

The concept of MANETs was originated from ALOHA proposed in the 1960s and the "wireless packet data network (PRNET)" project of DARPA [17], and then many projects such as the "viable adaptive network" and the "Global Information System (GloMo)" pushed the MANET development into a new step [18]. Until the 20th century, self-organizing networks were applied in the civilian system.

MANETs are special kind of networks composed of many mobile terminals, which have wireless transceiver capabilities. This class of networks does not need to rely on network infrastructures to quickly implement networking and communications. Wireless links between different nodes can communicate directly or indirectly to complete the information exchange.

The main features of MANETs are concluded in below [19] [20] [21]:

- The variation of network topologies: Most of the wireless networks have been used in the mobile environment. Specifically, the mobile nodes in MANETs can cause the entire network topologies constantly to change. Thus MANET routing protocols, security policies and other MANET technologies should adapt to the variation of topologies of MANETs.

- The security of MANETs is not very reliable: Comparing with the fixed telecommunication networks, nodes in MANETs can dynamically join and leave 
the networks at any time. Thus the node mobility feature enables the MANETs more vulnerable to be attacked, such as eavesdropping and intrusion. The MANET security issue is one of the important aspects in ad hoc network researches.

- Self-organization: MANETs can automatically form self-organized networks without individuals' intervention. Meanwhile, node can automatically reorganize when MANETs encounter errors or node failures.

- Multi-hop routing: Each node in MANETs is independent because of the limited communication distance, thus the communication between two non-neighbor nodes needs to implement multi-hop routing. In other words, if a node in outside of the effective range of communication needs to forward packets, this node must go through intermediate nodes to complete the routing path. This mechanism is called multi-hop routing.

- Communication distance is limited: Since each node in MANETs is self-existent, there is no fixed centralized control, together with the energy restrictions of each independent terminal node, all these characteristics make the transmission power of each node in MANETs small, so that the network nodes in an isolated geographical position are not able to communicate with each other.

- Effective network time is limited: Since MANETs often build communications because of some temporary reasons, thus the existence time cycles of entire MANET environment are short, so MANETs cannot maintain stable wireless network environment for a long time. 


\subsubsection{Software-defined Networking}

Since the individuals' demands continue to increase, the requirements of network equipment to meet the individuals' needs become higher and higher. Meanwhile, the increasing complexity of network equipment not only greatly improves the operation and maintenance costs, but also hinders the innovation of network devices. Due to current situations, researchers propose a novel network structure called SDN.

The thought of SDN aims to propose a new network technology in order to break through the limitations of traditional TCP / IP structure. SDN concept is better able to adapt to the rising technologies compared with the traditional networks. Dr. Martin Casado had proposed Ethane architecture [22] that used a centralized controller to send instructions to other network devices in enterprise networks. This mechanism completely controls and manages network resources. Inspired by this project, Professor Nick Mekeown found that the data planes and control planes of conventional network devices are separated from each other using a centralized controller and standardized interfaces for control and management of network devices. This method can provide a more flexible way for network management and can vigorously promote the performance of networks. In 2008, they proposed OpenFlow protocol [23], the core technology of OpenFlow is that it separates the forwarding planes from the control planes of traditional network equipment. In other words, OpenFlow protocol operates through a centralized controller and standardized interfaces to manage and configure a whole network. This thought established the prototype of SDN [24].

SDN can be considered as an emerging network architecture, which has been jumped out of the traditional network hardware restrictions. SDN can quickly adjust, expand and upgrade the network infrastructures. When deploying new services, network devices do not have to be configured individually. It is possible to reduce the time to build and deploy the new services, so SDN improves the network utilization 
effectively.

\subsection{Thesis Contributions}

As we mentioned before, there are many factors that significantly influence the MANET performance. This thesis mainly focuses on the software-defined mobile ad hoc networks with trust management in order to improve MANET performance. We first introduce the main features of SDN and MANET technologies, and then we propose a MANET trust model and an idea of the combination method of SDN and MANETs. Finally, we discuss the simulation results. The main contributions of this thesis are summarized as follows:

- With the recent advances in SDN, we propose a novel software-defined trust based mechanism in MANETs. Specifically, we separate the forwarding plane in MANETs from the control plane, which is responsible for the control functionalities, such as routing protocols and trust management in MANETs. As AODV [15] is one of the most popular routing protocols in MANETs, we use AODV as an example to implement our proposed SDN-base framework in MANETs called SD-TAODV.

- We divide the SD-TAODV into three layers, including MANET network layer, network operating system (NOS) and application layers. In addition, we move the AODV control logic and the trust management into the control node. This scheme aims to improve the network flexibility and management.

- We build the SD-TAODV system operating environment to achieve SD-TAODV prototype and test its functionality. 


\subsubsection{Accepted and Submitted Papers}

The following paper has been accepted:

1. Dajun Zhang, F. R. Yu, and Zhexiong Wei, "Software-defined Vehicular Ad Hoc Networks with Trust Management," in Proc. ACM on Design and Analysis of Intelligent Vehicular Networks and Applications (DIVANET'16), Malta, Nov. 2016.

The following paper has been submitted:

1. Dajun Zhang, F. R. Yu, and Zhexiong Wei, "Software-defined Vehicular Ad Hoc Networks with Trust Management," submitted to Ad Hoc and Wireless Sensor Networks Journal, Sept. 2016.

\subsection{Thesis Organization}

The rest of the dissertation is organized as below:

- Chapter 2: In this chapter, entitled "Overview of MANETs and SDN", we first describe MANETs and its key technologies, including theory and characteristics of the architecture; Meanwhile, we simply depict some representative MANET routing protocols. Secondly we introduce SDN and its northbound and southbound API; then we describe the OpenFlow key technologies, including standard protocols, controllers, and switches information exchange, matching flow table.

- Chapter 3: In this chapter, entitled "MANET Routing Problems and Related works", we describe the problems of MANET routing protocols. And then we introduce some recent works about the trust based MANET routing protocol mechanism.

- Chapter 4: In this chapter, entitled "Trust Based AODV Routing Protocol (TAODV)", we describe the AODV routing protocol in detail, and then we 
present the route discovery and route maintenance process of our proposed TAODV mechanism.

- Chapter 5: In this chapter, entitled "Software-defined Mobile Ad-hoc Networks based on TAODV", we detailedly describe fusion method of SDN and MANETs. First we describe the separation of the control and forwarding logic, and then we introduce the working process of controller. Finally the working process of forwarding node is presented in the last part of this chapter.

- Chapter 6: In this chapter, entitled "The Simulation Results and Discussions", we simulate our proposed scheme, and evaluate the simulation results. The simulation results show that our proposed SD-TAODV system can improve the network performance significantly.

- Chapter 7: This chapter summarizes the conclusion of the thesis and highlights the next step for future works. 


\section{Chapter 2}

\section{Overview of MANETs and SDN}

SDN is seen as a novel network architecture, which changes the traditional network frames, applications and managements. In order to get rid of the restrictions on network hardwares, SDN can easily adjust, expand and upgrade to the network frame. For the MANETs, there are also network control and management issues, so we can refer SDN technology to optimize the network control and management of MANETs.

\subsection{Key Technologies of Mobile Ad Hoc Networks}

\subsubsection{Ad Hoc Theory and its Characteristics}

After decades of development, ad hoc networks have gradually developed a theoretical system. MANETs use inherent mechanisms to form complete and complex self-organized systems. There are some rules that need to be followed as the network running: 1) each node in the networks has a criterion for the whole network; 2) each node has its own capacity to implement decisions; 3) each node determining their own behaviors will also affect the state of whole networks; 4) all nodes in the networks operate in the form of parallel [25].

Each node in MANETs can act as a router or a host, which means that either a 
node can run as a host to operate user-oriented applications, or it can run as a router to operate the routing protocols such as AODV and DSDV. Meanwhile, nodes can forward packets and operate routing maintenance according to the routing policies.

Ad hoc applications are mainly related to its own characteristics. Compared with the conventional wireless network communication environments, MANET application fields mainly show its self-organization, rapid deployment, and dynamic topology features [26] [27].

- Military: Ad hoc nodes have the features of rapid networking and high mobility, so MANETs are frequently utilized in the military field. Nowadays, soldiers, military vehicles and large equipment are all required to maintain real-time contacts. However, because of the special natures of the battlefields, the battlefields are difficult to build fixed wireless communication environments, so the ad hoc networking is a kind of preferred wireless networks for commanders to establish communications.

- Sensor networks: Sensor networks are mainly used in wireless communication, but due to the small power of the sensor itself and some other reasons, the sensor networks can use ad hoc technologies to form multi-hop mechanism to complete the communications. The combination of MANETs and sensor networks has a very good prospect in the future.

- Disaster environments: When happening some devastating natural disasters such as earthquakes and floods, the existing fixed communication devices cannot complete the communications, so individuals need to quickly establish networks for communications to execute the rescue works. Ad hoc networks enable people to quickly build good communication network environments in such disaster sites. 
- Establishing networks in remote areas: When staying in some remote areas, people have to face an environment of non-fixed wireless network infrastructures. If individuals need to build networks, they can take advantage of ad hoc networks to rapidly deploy the efficient wireless communication environments.

- Personal communication applications: Nowadays, PAN (Personal Area Networking) attracts more and more individuals' attention. In order to freely communicate between personal devices and to solve the energy consumption of personal communication devices, people need to establish a wireless communication environment that uses multi-hop ad hoc mechanism to meet the communication needs of individuals.

- Combining with the traditional wireless networks: Ad hoc networks can combine with the traditional fixed network environments. Traditional wireless communication networks can maintain high quality communication environments over a wide range area for a long time, and ad hoc technologies can further expand the local areas of communication networks. The functionality of multi-hop can extend the effective communication distance of traditional wireless networks.

\subsubsection{The System Architecture of Mobile Ad Hoc Networks}

According to the characteristics of MANET and the traditional TCP / IP structure, we also divide the MANET structure into five layers:

Application layer: Application layer includes different kinds of the functions such as real-time operation and data traffic.

Transport layer: The transport layer operates the TCP and UDP.

Network layer: The network layer includes two sublayers: network interconnected layer and network layer. The network interconnected layer operates IPv4 or IPv6. The network layer is used to operate routing protocols of MANETs. 


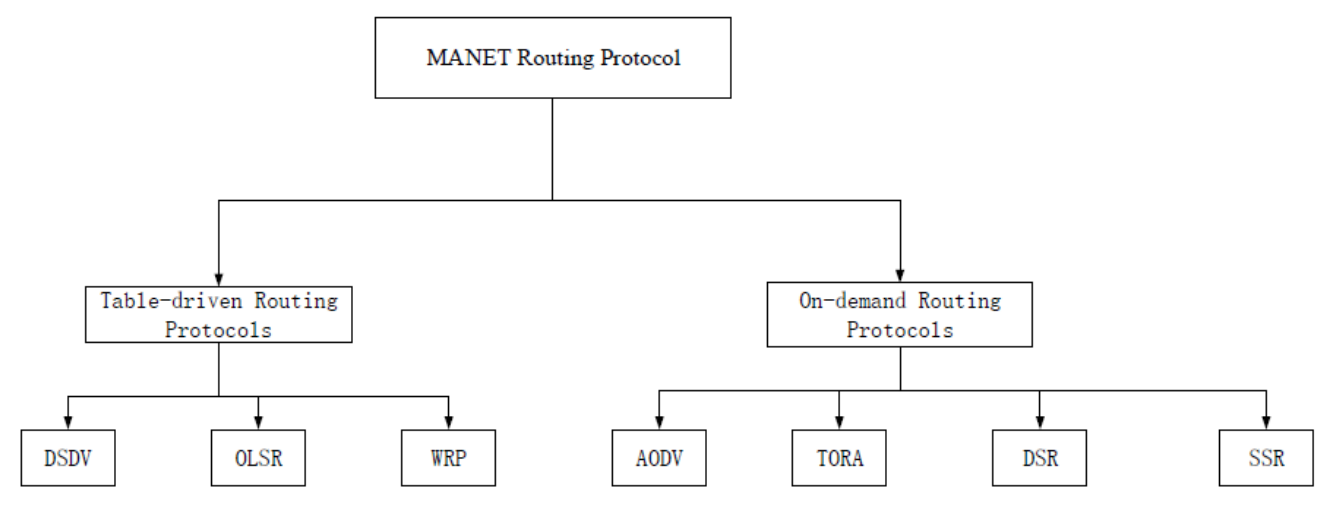

Figure 2.1: The MANET routing protocols [1].

Data link layer: The data link layer can be divided into two sublayers: logic link control (LLC) layer and media access control (MAC) layer. The data link layer is responsible for data frame detection, packets confirmation and error control. In this thesis, we use IEEE 802.11 as our protocol of MAC layer.

Physical layer: The main technologies of physical layer are phys frequency selection and detection, modulation and demodulation, etc.

\subsection{The Categories of MANET Routing Protocols}

MANETs require routing algorithms to adapt to their unfixed network topology feature in order to avoid ring paths and to reduce overhead. This requirement makes the network nodes have a ability to justice the current state of the networks. According to the route discovery strategy, MANET routing protocols can be divided into two categories: proactive routing protocols and on-demand routing protocols as shown in Figure 2.1. 


\subsubsection{Proactive Routing Protocols}

In the proactive routing protocols, each node maintains its own routing table, which contains the routing information from one node to other nodes. Nodes periodically broadcast routing information packets to exchange routing information in order to maintain their own routing table. The routing table is updated periodically. When a node needs to send a packet, it can quickly find appropriate routing path(s) according to the routing information in the routing table. One of advantages of proactive routing protocols is that when an intermediate node needs to build communication with another node, a appropriate routing path always can be immediately found. However, table-driven mechanism also brings some disadvantages. For example, networks are required to spend a lot of overhead to maintain routing tables. This disadvantage results the waste of network resources. This kind of routing protocols mainly include DSDV, WRP, OLSR, and so on.

- DSDV

DSDV [28] [29] (Destination Sequenced Distance Vector) protocol is modified through the existing routing protocol RIP. DSDV protocol adapts to the ad hoc wireless network requirements. In this protocol, each node maintains a routing table, which has a routing information of all neighbor nodes connected to this node. One of the routing entries includes: destination node, hop count, next hop node and sequence number. Specifically, sequence number is used to justice whether the routing message is the outdated information and to prevent routing loops. DSDV has common features of distance vector routing protocol, it periodically updates routing information. Therefore, DSDV has slow convergence, but unlike other routing protocols, DSDV introduces a sequencing mechanism in order to prevent routing loops, thereby reduces the load of routes, so that 
route convergence time has certain reduced. In DSDV, if a network is largescale, the network overhead is enormous. Similarly, DSDV does not appropriate for the fast-moving network environment.

\section{- WRP}

WRP [30] [31] (Wireless Routing Protocol) is a kind of proactive routing protocol. Each node in WRP must maintain four tables: routing table, distance table, link cost table and message retransmission list, routing table records the destination information, predecessor information and subsequent node information, this recording precursor and subsequent information mechanism effectively prevent routing loops. Distance table records the distance from each node to the destination via the intermediate nodes, and the table also contains the next hop information. Link cost table records the cost of each node to its neighbors. Message retransmission table records the information about retransmission message, which includes sequence number, the times of retransmission, ACK flag, and updating message list. A node communicates with its adjacent nodes periodically. The node communication includes routing tables updating and link information exchanging. This mechanism obtains the status of neighbor nodes so that nodes can maintain their own routing table. If adjacent nodes do not send packets or update messages, the nodes send Hello messages to ensure connectivity. In WRP protocol, each node stores information of its neighbors, so any node in WRP can establish relative optimize paths. However, since each node in WRP protocol needs to maintain four information table, WRP requires more memory and needs to maintain additional information, which means the greater network overhead.

\section{- OLSR}


OLSR [32] [33] (Optimized Link State Routing) protocol is optimized from the traditional LS (Link State) protocol. OLSR aims to adjust the ad hoc wireless network requirements. In this protocol, each node periodically exchanges information with its neighbors in order to maintain network topology. The key concept of OLSR is multi-point relays (MPR), which reduce the scope of the flooding. Each node selects part of its neighbors as the multi-point relay nodes. When a node sends control packets to all its neighbors, only its MPR nodes forward control packets, and the other neighbor nodes are not able to forward the packets. Thus, the MPR mechanism reduces the number of network packets forwarding, and it can effectively decrease the flooding.

In summary, proactive routing protocols exchange routing information periodically and maintain network topology and routing information, thus the route discovery delay is small. However, proactive protocols need a lot of packets, which means that the protocols enlarge the network overhead. So the proactive protocols are not suitable for the situation that the network topologies always change. In order words, the network control information in this situation will exponentially increase, so that makes the network overhead increase significantly.

\subsubsection{On-demand Routing Protocols}

Reactive routing protocols [34] [35] also known as the on-demand routing protocols, nodes running at this kind of protocols need to find and establish the routes only when the nodes have demands for transmitting data. This kind of protocols is different from the table-driven routing protocols. Reactive routing protocols do not require to exchange the routing information periodically. Generally, this kind of protocols includes route discovery process and route maintenance process. When a source node sends a packet, and the node routing table does not have a route to destination, 
the source initiates the route discovery process. The source node broadcasts request packets to the whole network topology. When the route request packets arrive at the destination node, the valid route is established. The destination node sends a route reply packet to the source in order to establish a forwarding route. The source node then transmits data packets along the established routing path. If the path breakdown, the source node initiates the route maintenance process to restore the route. The features of the reactive routing protocols determine that the network overhead is small, but latency is large. This kind of protocols is not suitable for real-time needs. Reactive routing protocols include DSR, AODV, TORA, etc.

\section{- DSR}

The feature of DSR (Dynamic Source Routing) protocol [36] is the source routing mechanism, which means that packet headers contain the routing information about source node to destination node, nodes in DSR send packets according to the packet header information. DSR includes route discovery and route maintenance process. When a node needs to send data packets, first it checks its own routing table whether exits a route to the destination node. If so, source transfers data packets as usual. If not, source initiates the route discovery process. The source node uses flooding method to broadcast route request packets (RREQ), when a node receives a RREQ packet, it first determines whether it receives the same RREQ before, if so, the node discards this RREQ packet; if not, it determines whether the current node is the destination. If this node is the destination, it returns a route reply packet (RREP); if not, the node checks its own routing table if there is a route to the destination node. If the available routes exist, the node responds a RREP packet; if not, the node continues to broadcast the RREQ packet to its neighbor nodes. If a routing path break down, the source node initiates the route maintenance processes. The features 
of DSR ensure a loop-free routing path, and nodes only need to maintain the routing information of their connected nodes, rather than the whole network routing information. This mechanism reduces the network overhead, but the head of the data packet must carry the entire route information, which makes the data packet overhead become large. Meanwhile, the flooding method of the route request packets may result unnecessary network overhead.

\section{- TORA}

TORA [37] is a distributed routing algorithm. When the network topology changes, a control packet is positioned within the range of the local network topology, so that nodes maintain routing information only with their neighboring nodes. In addition, except for the route discovery and route maintenance process, TORA routing protocol also includes the route removal process, in which uses flooding manner to broadcast CLR (clear) packets to remove the invalid routes. TORA mechanism is more like the process of water flowing from the top of mountain to the foot of the mountain through the pipeline. Data packets or control packets like the water; pipeline like the links between the nodes; each connection point between the pipes are the same meaning with the nodes in the network. Each node in the routing algorithm calculates a height to control the Packets flow from "high" to "low". The CLR packet that has a relatively high value is forwarded to a node having a relatively low value. When a node detects a link broken, the node improves their height values to a more higher level than the height of its neighboring nodes so that makes the "water" reverse, thus makes the "water" flow to the destination node through other routes. The algorithm can find multiple routes from the source node to destination. TORA protocol has strong adaptive ability, high efficiency, and the good scalability, these features make it more suitable for high-speed, dynamic 
topology, and multi-hop wireless networks.

From the several representative reactive routing protocols, this kind of routing protocols do not maintain the network topology continuously. They do not maintain the entire network routing information, and only maintains the routing information that the nodes required to build communication. This feature reduces network overhead, but the delay of the on-demand route discovery greatly increases.

\subsubsection{AODV Routing Protocol Introduction}

AODV is one of the most widely used routing protocols in MANETs [16]. A unique characteristic of AODV is that the routing protocol introduces "sequence number" concept, which is utilized to avoid the count to infinity problem and to prevent rooting loop [38]. Specifically, each node in AODV must maintain its own routing table that includes routing information about its neighbor nodes. The operating procedure of AODV can be divided into two main operations: route discovery and route maintenance [39].

Route discovery process is initiated by a source node if the source node needs to send data packets to a destination, but there are no valid routes in the routing table of the source node. So the source node first broadcasts RREQ packets to its neighbors. A RREQ packet contains the following fields: originator IP address, originator sequence number, destination IP address, destination sequence number, and hop count [16]. There are two different situations when a node receives a RREQ packet: i) this node sends a RREP packet if the node is the destination or it knows the route(s) to the destination; ii) the receiving node establishes a reverse route to the source if the routing table of this node does not have a routing entry to the destination. Figure 2.2 shows the frame of a RREQ packet.

Type: Type field represents the frame type. RREQ frame is set to 1. 


\begin{tabular}{|l|l|l|l|l|l|l|l|}
\hline Type(8 bit) & J & R & G & D & U & Reserved (11 bit) & Hop Count (8 bit ) \\
\hline \multicolumn{4}{|c|}{ RREQ ID } \\
\hline \\
Destination IP Address \\
\hline \\
Destination Sequence Number \\
Originator IP Address \\
\hline \\
\hline
\end{tabular}

Figure 2.2: The frame of the RREQ packet.

$J: J$ tag is used to add a tag to achieve multicast reservation.

$R$ : Repair tag is used for the realization of multicast reservation.

$G$ : Gratuitous RREP flag is used to justice whether it generates a free RREP mark to the destination node. If an intermediate node responses RREQ, $G$ flag sends a RREP frame including this node IP address to the destination at the same time. This RREP frame is named free RREP frame.

$D$ : It points out that only the destination node can respond to this RREQ.

$U$ : It is used to identify unknown sequence number.

Reserved: Sent as 0; ignored on reception.

Hop Count: The number of hops is calculated by a RREQ packet from the source node to the destination node.

RREQ ID: The ID of RREQ frame.

Destination IP Address: The IP address of the destination.

Destination Sequence Number: The sequence number of the destination node. Each node updates its own sequence number if it receives a better sequence number.

Originator IP Address: The IP address of the source node.

Originator Sequence Number: The sequence number of the source node.

After RREQ packets arrive at the destination, destination node unicasts a RREP packet to the source node along the selected reverse path. The RREP message includes the following fields: hop count, destination IP address, sequence number, 


\begin{tabular}{|c|c|c|c|c|c|}
\hline Type (8 bit) & R & A & Reserved (9 bit) & Prefix Sz(5 bit) & Hop Count (8 bit) \\
\hline \multicolumn{4}{|c|}{ Destination IP Address } \\
\hline Destination Sequence Number \\
Originator IP Address \\
\hline Lifetime
\end{tabular}

Figure 2.3: The frame of the RREP packet.

lifetime, and originator IP address [40]. Figure 2.3 shows the RREP packet frame. When an intermediate node receives the RREP message, it sets up a forwarding route entry to the destination, and continues to send this message to next hop. This procedure will continue until the RREP packet arrives at the source node. When the source node receives the RREP message, the route discovery procedure end, and then data packets are sent to the destination by the source node along the forwarding route entry direction. Figure 2.4 shows the route reply process.

Type: Type field represents the frame type, RREP frame is set to 2 .

$R$ : Repair mark is used for multicast reservation.

$A$ : If the RREP information is already set "A", the nodes which have already received the RREP packets need to return RREP-ACK information.

Reserved: Sent as 0; ignored on reception.

Hop Count: The number of hops transmit from the source node to the destination node.

Destination Sequence Number: The sequence number of the destination node.

Destination IP Address: The IP address of the destination.

Originator IP Address: The IP address of the source node.

Lifetime: The time of a RREP message can be survived in the route discovery process.

Each node in AODV needs to maintain their own routing table, which includes: destination sequence number, IP address, hop count, next hop and lifetime [14]. If 

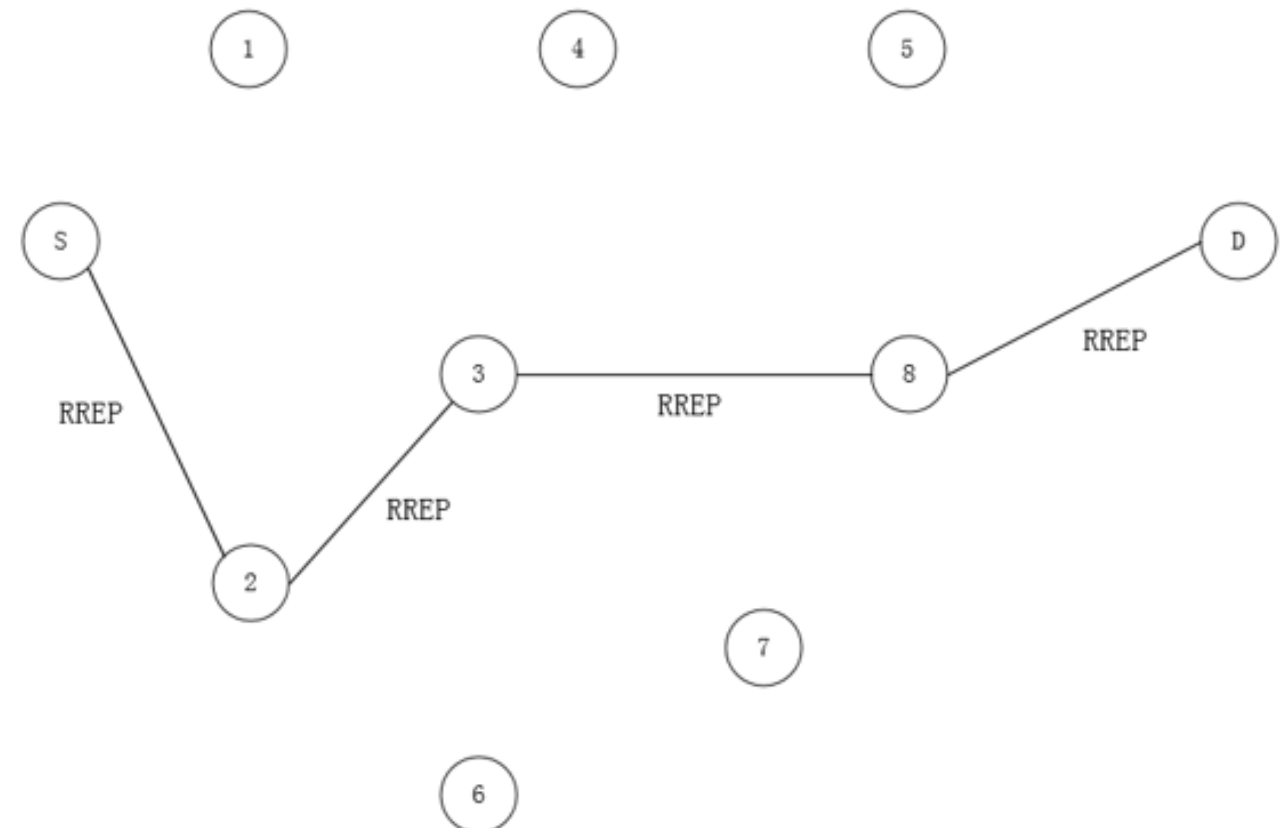

Figure 2.4: A route reply process.

the source node wishes to send data packets to the destination, first it needs to check its own routing table whether the routing table has a valid route to the destination. If so, source node sends data packets to its next hop. If not, the source node puts the data packets to its buffer queue and initiates the route discovery process, in which the source broadcasts RREQ packets to its neighbors. When a neighbor node receives a RREQ packet, it updates its routing table and establishes a reverse route to the source. Specifically, each node drops the RREQ packets only if it has already received the RREQ packet with same RREQ ID before. There are two situations that a node responds a RREP message to the source. Either the node is the destination or the node has a fresh route to the destination [41]. Figure 2.5 shows the route discovery process, where $S$ is the source node and $D$ is the destination node, the red lines denote the reverse routes to the source.

The route maintenance procedure is operated by nodes in two different ways. One situation is that a node broadcasts hello messages to its neighbors at regular 


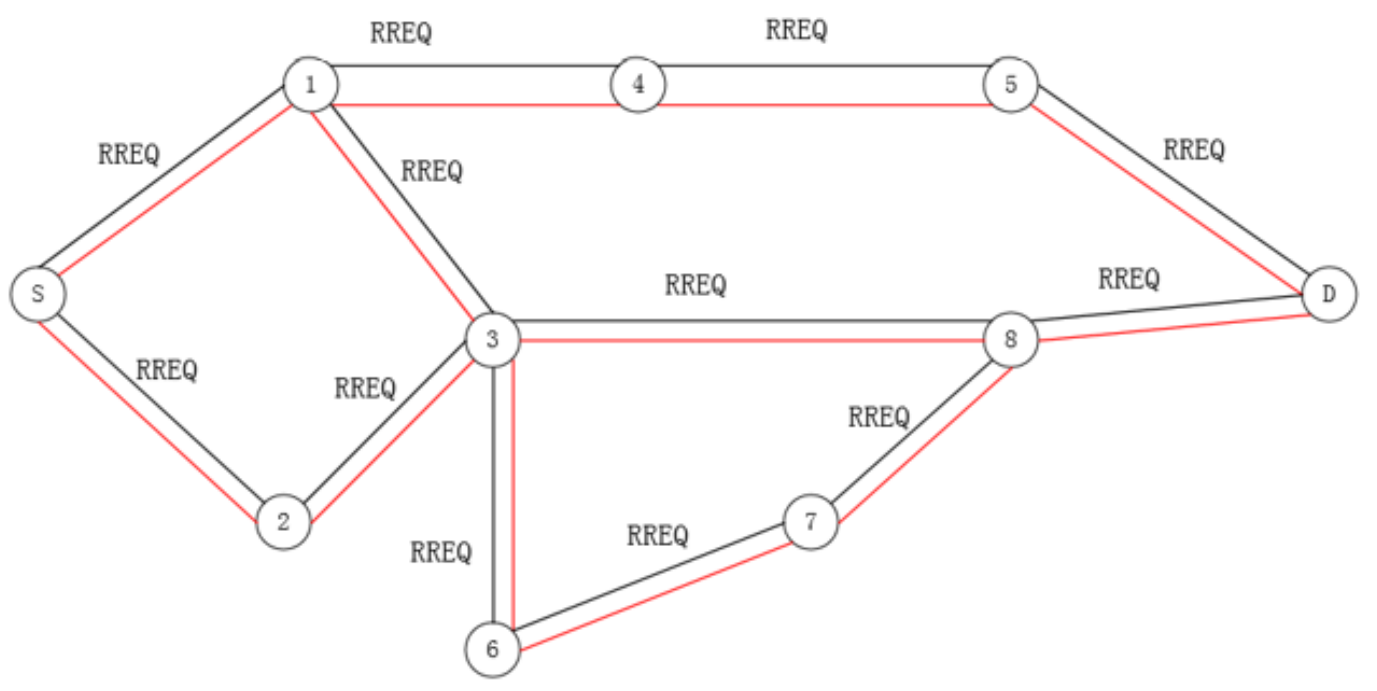

Figure 2.5: Route discovery process.

time intervals so that nodes can maintain connectivity with its neighbors. Another situation is that the procedure aims to increase the successful data transmission ratio through the local repair mechanism [38]. Because of the node mobility, a routing path between two nodes maybe breaks, so the local repair aims to recover the routing path. Conversely, if a node do not receive a RREP message during a period of time, the local repair fails and the node sends a request error (RRER) message [38]. A RRER packet contains a list of unreachable destination node information. The functionality of the RRER packet is that the packet can notify the source node to rebuild a new routing path if this source node still needs to transfer data packets.

\subsection{Software Defined Networking and its Inter- faces}

With continuous expansion of the scale of computer networks, routers running on the networks become increasingly complex. In TCP / IP network architecture, network devices (such as switches, routers, etc.) are generally composed of the control and 
forwarding planes. The control plane is responsible for the forwarding plane to determine the protocols, which include gateway protocols and routing protocols. The forwarding plane is an entity that operates the routing strategies established by the control plane, including data encapsulation, finding forwarding table, and package de-encapsulation, etc.

However, the control and forwarding planes of network devices are generally designed and developed by the equipment providers. A large number of highly integrated network protocols are concentrated on hardware, and the control planes and forwarding planes are all bundled in the network devices. Configuring and managing the networks are only achieved by people through a command line interface (CLI) [42]. CLI can individually configure a single network device, but CLI is hard for users to control the entire networks because the CLI is not user-friendly, which means that users need to remember a lot of commands and parameters. The users have no other ways to configure and manage the network than typing the complex and right commands. This control and forwarding plane tightly coupled way brings a series of problems in network management and deployment, such as the complexity of network management and low efficiency of the network functions. Therefore, SDN came into our eyes. In recent years, SDN as a future alternative network has attracted more and more attentions.

The framework of SDN is a separated architecture that control and forwarding plane are decoupled from each other. SDN operates through the standardized protocols like OpenFlow to control and manage the network devices [43]. As shown in Figure 2.6, the control plane of SDN is deployed on a controller, the controller intensively control the multiple network devices in network, so SDN improves the network flexibility [44]. Moreover, the control plane has a global view of the network, so SDN allows network administrators to configure the network effectively and to deploy 


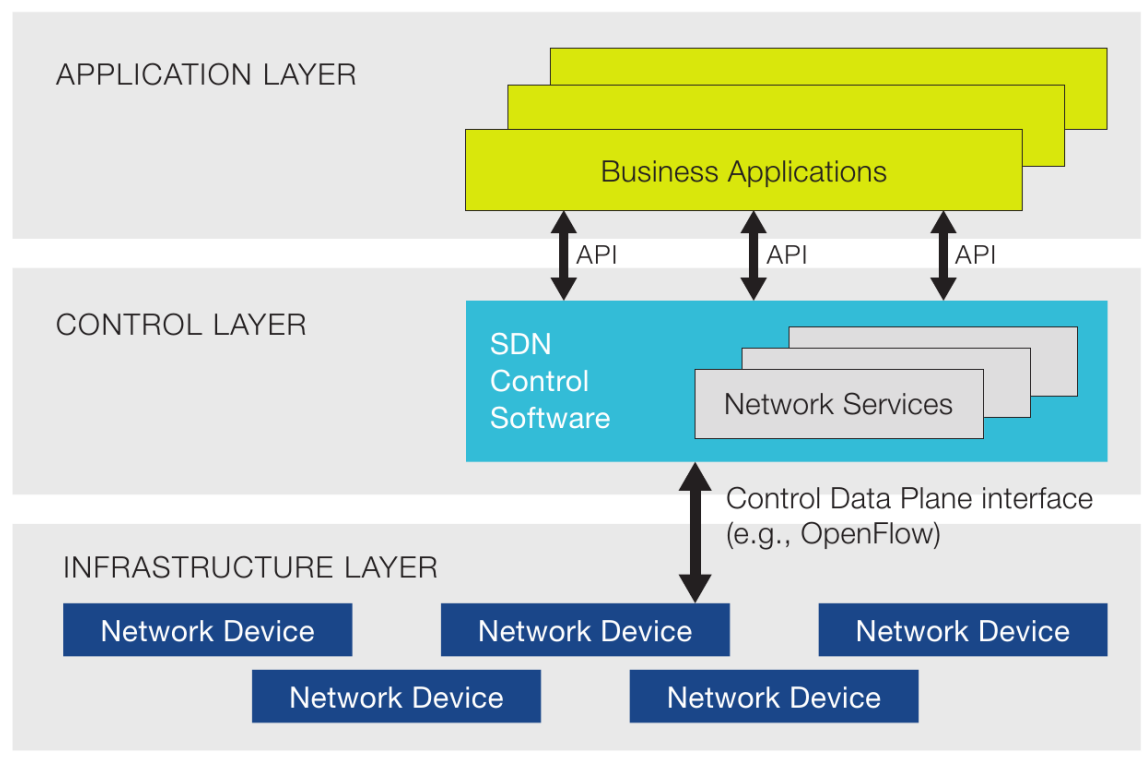

Figure 2.6: The SDN architecture.

various types of network applications easily.

There are many protocol standards on the use of SDNs in real applications. One of the most famous protocol standards is called OpenFlow [8]. OpenFlow is a widely used protocol that introduces the SDN concepts to implement in hardware and software. An important feature of OpenFlow is that scientists can utilize the existing hardware to design new protocols and to analyze their performance [45].

The frame of OpenFlow can be divided into three layers: network infrastructure layer, control layer, and application layer [46]. Infrastructure layer is composed of underlying network devices (such as OpenFlow switch), which are responsible for forwarding data packets. The control layer is the core layer of the OpenFlow framework, which is responsible for maintaining the entire network status, including network topology, node status, and link status information. Meanwhile, the control layer sends the matched flow table(s) to the underlying network devices through the southbound interface (data forwarding plane and control plane interface), as well as provides information to the upper layer through the northbound interface (control 


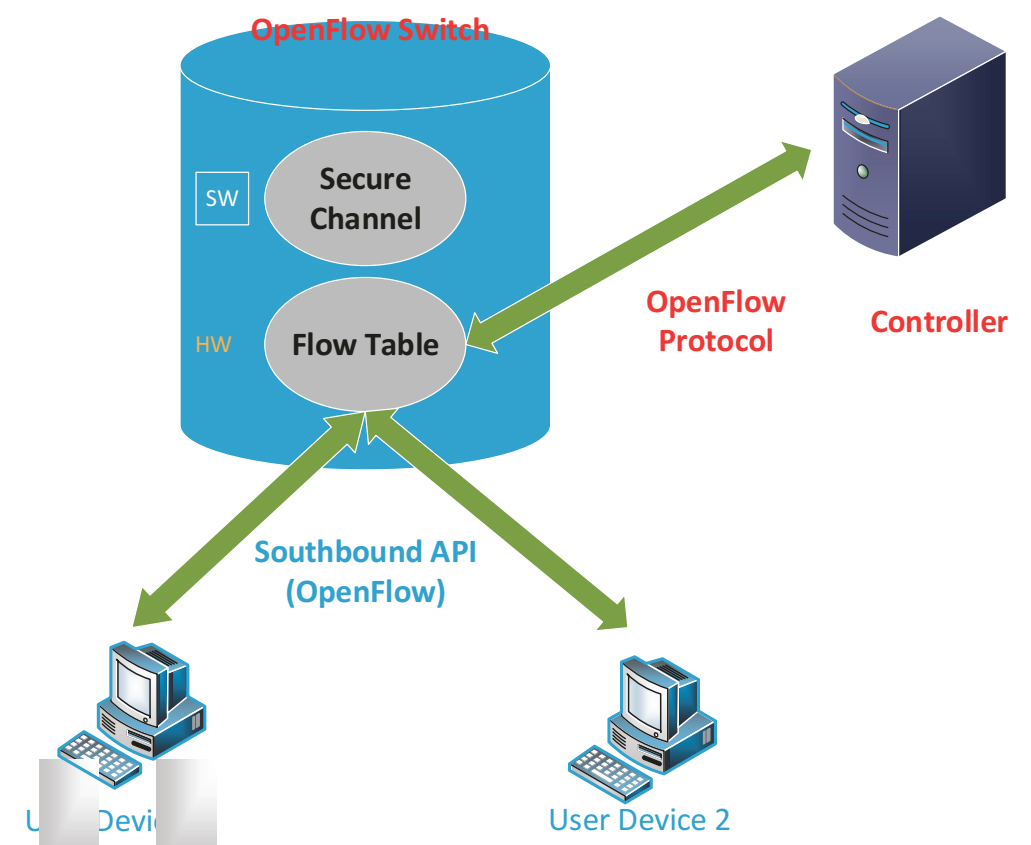

Figure 2.7: The OpenFlow switch and controller interaction using OpenFlow protocol.

layer and application layer interfaces). Application layer can be invoked through the northbound interface in order to communicate with the underlying network resources. Figure 2.7 shows an example of the OpenFlow architecture.

\subsection{OpenFlow Key Technology}

In order to achieve SDN requirements, academia and industries have made unremitting efforts to put forward a series of new technologies. OpenFlow technology is a model for the realization of SDN because of its technical specifications and scalability. OpenFlow is now widely accepted by researchers. Many network equipment providers, operators and software companies actively involve in the development of OpenFlow standards and a series of technical support for OpenFlow network devices. 


\subsubsection{OpenFlow Protocol}

The structure of OpenFlow has three important components: switches, controllers and flow-entries [8]. An OpenFlow switch at least includes three fields [47]: a) flow table(s), which each flow table has an action field associated with each flow entry; b) a communication channel, which provides links for the transmission of commands and packets between the controllers and switches; c) an OpenFlow protocol, which enables an OpenFlow controller to communicate with routers or switches. The controller can update flow-entries from the flow table(s) and can inform the switches what they can do in next step. In wireless environment, a static controller can be a brain of whole network to establish packet transfer paths. Most parts of the OpenFlow switches support three actions: a) sending flow packets to a port; b) encapsulating the flow packets and sending them to a controller; c) dropping the flow packets.

\subsubsection{The Information Interaction of OpenFlow Protocol}

The information interaction between OpenFlow switches and controller(s) supports three kinds of messages: controller-to-switch, asynchronous, and symmetric [23]. In version 1.3 of OpenFlow, controller-to-switch messages are shown in Figure 2.8. The most important message in control-to-switch is the OFPTFLOWMOD [48], which is used to modify the flow table in the OpenFlow switches.

Asynchronous messages are initiated by switches, the switches are used to update the status information to the controller. Asynchronous messages include four message types as shown in Figure 2.9. In these four asynchronous message, the most critical message is OFPTPACKETIN [23], which OpenFlow switches use this message to send packets to controller if the packets cannot be handled by the switches.

Symmetric messages are initiated by OpenFlow switches or routers. The messages include three types as shown in Figure 2.10. 


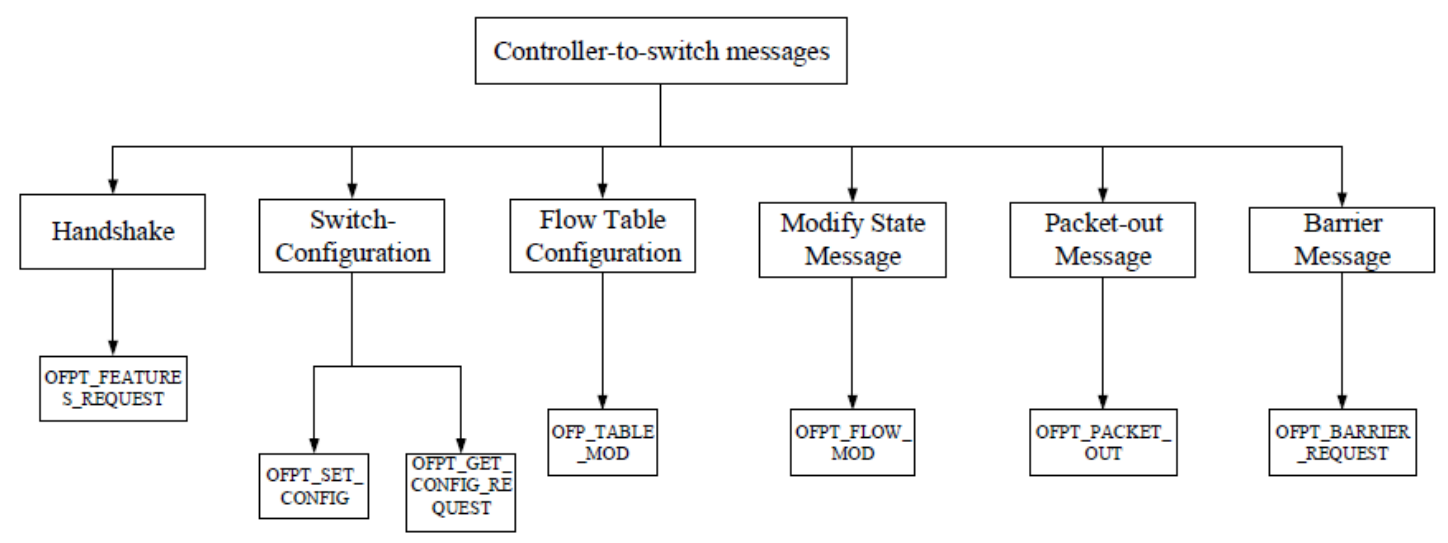

Figure 2.8: The controller-to-switch message.

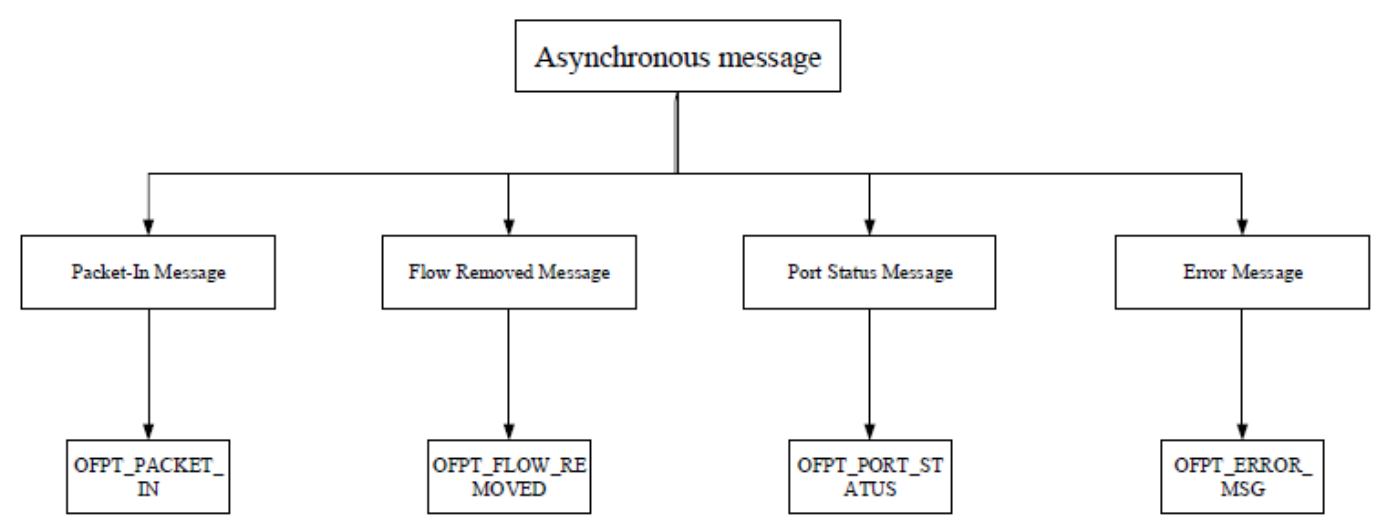

Figure 2.9: The asynchronous message. 


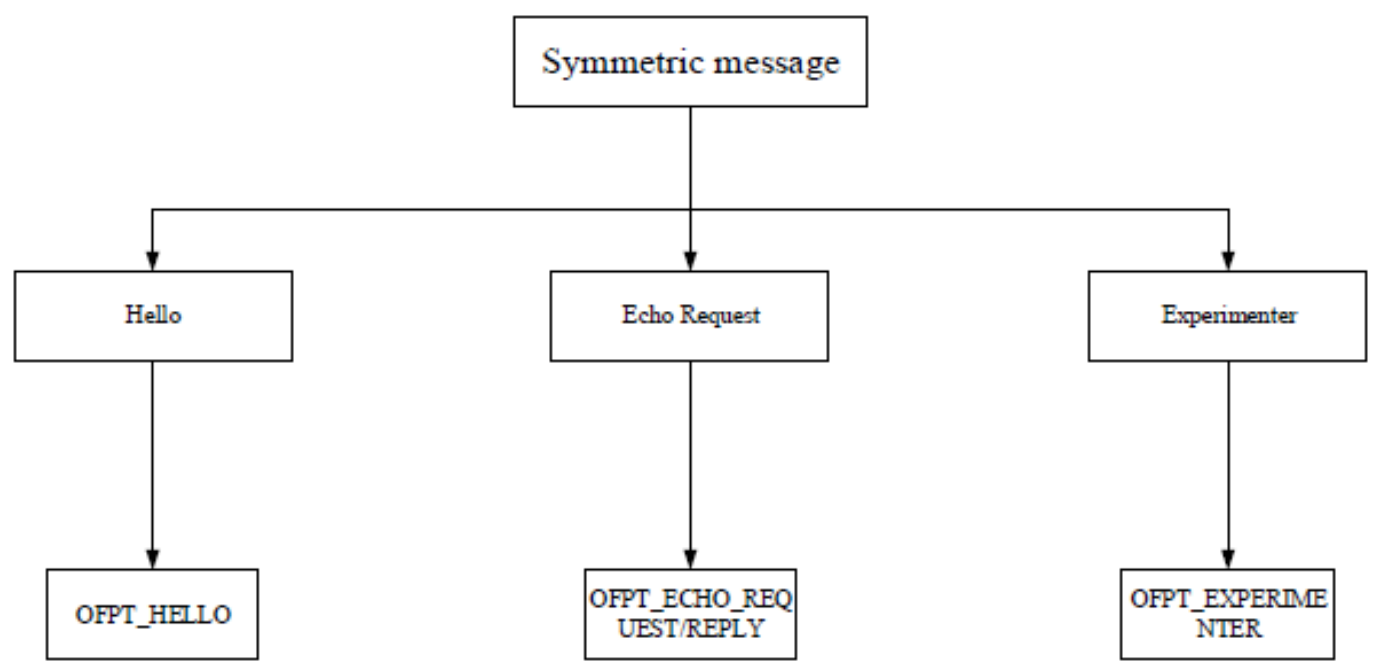

Figure 2.10: The symmetric message.

\subsubsection{The Matched Process of Flow Tables}

The core processing unit of an OpenFlow switch is flow table, each of the switches includes a flow table, and the switches can communicate with the controller via a secure channel, and the flow table updates via OpenFlow protocol. The OpenFlow switches establish flow table entries to forward data. In the OpenFlow version 1.3, a flow table entry contains six parts: matched fields, priority, counters, instructions, timeouts, and cookie [49].

- Matched fields: OpenFlow 1.3 provides 40 matching fields, including 13 require fields and 27 optional fields. Required fields include [50]: the switch port (INPORT), the Ethernet destination MAC address (ETHDST), the Ethernet source MAC address (ETHSRC), Ethernet type (ETHTYPE), IPv4 or IPv6 protocol number (IPPROTO), IPv4 source address (IPV4SRC), IPv4 destination address (IPV4DST), IPv6 source address (IPV6SRC), IPv6 destination 


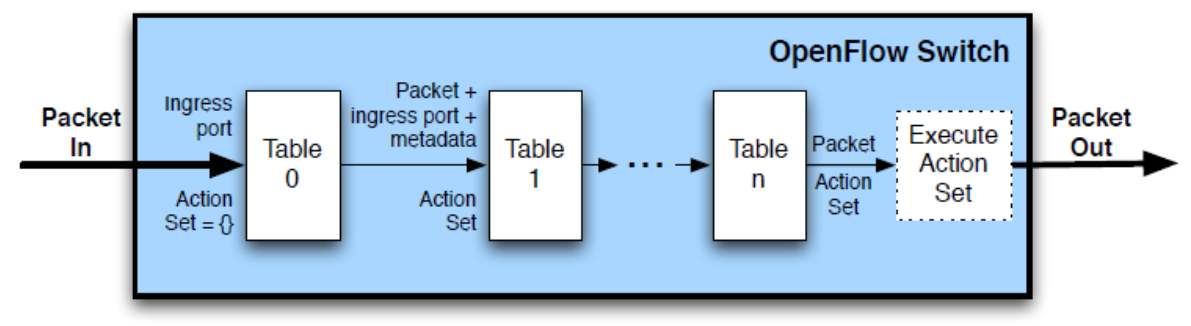

Figure 2.11: The matched process of OpenFlow switch.

address (IPV6DST), TCP source port number (TCPSRC), TCP destination port number (TCPDST), UDP source port number (UDPSRC), UDP destination port number (UDPDST).

- Priority: priority field is used to indicate the matched sequence of flow entry.

- Counters: to update the matching packets.

- Instructions: to modify the action sets in flow tables or pipeline processing.

- Timeouts: timeouts is used to record the maximum time before flow is expired by the switches.

- Cookie: maybe used by the controller to filter flow statistics, flow deletion and flow modification, and not used when flow tables process packets.

After an OpenFlow switch receives a data packet, the matching process of flow tables is shown in Figure 2.11. The matching process is begin from flow table 0 and until the packet matches the corresponding matched field, and then the action set of this matched field is operated. If data packets cannot find appropriate fields, the packets are discarded or uploaded to the controller to process through the OFPTPACKETIN message. 


\section{Chapter 3}

\section{MANET Routing Problems and Related}

\section{Works}

In this chapter, we simply describe the problems of the existing MANET protocols. Meanwhile, we discuss and review the related works in the literatures about the trust based schemes.

\subsection{The Problems of the MANET Routing Proto- cols}

In MANETs, each node is both host and router, exchanging information between the nodes aims to maintain the network topology and routing information. The purpose of MANET routing protocols is to ensure that data packets can be safely and properly transmitted to the destination node. However, the inherent characteristics of ad hoc networks determine that MANETs are easy to be attacked by a variety of credible threats, which affect data packets correct forwarding. In general, the following aspects are the main problems of MANETs [51] [52] [53].

- Routing protocol technologies 
The problems of MANET routing protocols are one of important parts in ad hoc network researches. In terms of the routing, the prominent feature is dynamic and distributed network topologies, but this feature is a big challenge to design protocols in MANETs. The existing routing protocols of MANETs may not meet the real-time dynamic feature of ad hoc networks. Consequently, welldesigned routing protocols are the arduous tasks for researchers to improve MANET performance.

- Access control technology

In ad hoc networks, each individual node has autonomous mobile features, while the communication range of each node is limited by its own energy and external conditions, so researchers have to design appropriate MAC protocols to resolve channel problems. For example, channel utilization needs to increase and the network end-to-end delay needs to reduce.

- QoS technology

Currently, ad hoc wireless communication resources may not meet the growing communication needs. For example, large-scale data transmission has a high requirements for the bandwidth of the wireless channel. Meanwhile, the transmission delay and transmission speed of MANETs also have corresponding requirements. In order to ensure efficient transmission of massive amounts of data, QoS techniques have been proposed for the efficient information interaction in the ad hoc networks.

- Energy restriction

Each independent node in MANETs is often limited in energy. The limited energy feature has become one of the main problems of MANETs. The energy consumption of mobile nodes makes the nodes' transmitting power decrease, 
which results the mobile nodes invalid and affects the network topologies, evenly may cause network paralysis. Therefore, designing MANET routing protocols must take into account the energy consumption of nodes.

- Network security

Comparing with the traditional fixed communication networks, MANETs have the features of node mobility and open links. These features also make MANET safety cannot be well protected. MANETs are easier attacked by attackers, such as eavesdropping, masquerading and other malicious attacks. Meanwhile, nodes freely join or leave the networks make the security maintenance of ad hoc network become more difficult.

- Resource management

Ad hoc network resource management is a very important and difficult technique. On the one hand, in order to guarantee the quality of service of data processing, we need to manage the radio resource efficiently; on the other hand, we have to solve the problem of rapid changing topology of MANETs. Moreover, since the mobile nodes' energy is limited, power management must be a holistic approach in order to obtain reasonable optimization results.

- The network topology management

Ad hoc networks have multi-hop and self-organization characteristics, so there are more potential problems in network management compared with the traditional fixed and centralized control network. For example, the positioning of mobile nodes has a lot of problems; in addition, how to quickly detect the dynamic changing in MANET topologies also needs to be solved. Meanwhile, ensuring rapid responses for the network services are also one of the MANET problems that needs to be solved in the future. 
In summary, there are still some problems that need to be solved in MANETs. Specifically, we mainly focus on the two problems of MANETs in our thesis: one is the network security problem, another is the network topology management. The bad behaviors of nodes brings a lot of problems, including data services cannot be sent correctly, bad nodes degrade MANET performance. How to add security mechanisms in the routing protocols to distinguish and to detect the malicious behaviors of nodes is important for MANET to enhance the network performance. Ensuring the reliable delivery of data services has become a major direction of the MANET researches. With further researches, in order to solve this problem, the concept of reliability and credibility of the route have been proposed, and attracts great attention of the domestic and foreign scholars.

\subsection{Related Works}

In [54], the authors propose two mechanisms: watchdog and path rater, and use these as DSR protocol extension. Credible watchdog mechanisms need to assume that the nodes need to turn promiscuous mode, which has become a basic assumption of trusted based MANET routing protocols. Watchdog identifies misbehavior of nodes and monitors the received packets to get the trust value. Path rater rates the node according to the reliability of nodes. These two mechanisms can effectively find credible path. The results of the watchdog and path rater mechanism are all better than the Intrusion Detection System. However, in this paper, the authors only used the unicast MANETs and does not evaluate the performance of security techniques to other types of attacks.

Protocol ARAN [55] provides a single path routing solution in a safety management environment. It uses pre-defined encryption certificates to provide authentication and non-repudiation services, thus this mechanism ensures the end-to-end 
certification. However, ARAN protocol results in a large route discovery delay and overhead.

Protocol [56] adds a simple packet acknowledgment mechanism, which includes PKT and ACK packets. The protocol uses Merkle Hash Tree method to implement the validation work of PKT packets efficiently. After verification at the destination node, the node sends ACK message. Through these mechanisms, the protocol achieved good scalability.

Protocol LTB-AODV [57] mainly considers the types network attack. Each node turns the promiscuous mode, and each node monitors the behaviors of its neighbors. This paper mainly focuses on two types of attacks: black-hole attack and grey hole attack. In this method, the indirect credibility value is obtained by calculating the average recommendation values of neighbors. The protocol uses a fully distributed algorithm, and only uses information within one hop, so this protocol has a very good scalability. However, this protocol requires each node to listen and save almost all its neighbors communication record, the memory overhead of the protocol is big. Meanwhile, the lack of trust maintenance largely affected the performance of the protocol.

Protocol AOTMDV [58] uses a historical behavior of nodes. The authors propose a credible mechanism mainly based on fuzzy logic prediction method. The protocol can find the shortest paths that nodes meet the minimum requirement of the node trust value. In order to maintain trust value, this protocol uses a routing update reporting mechanism, the amount of changing trust value exceeds the threshold will trigger the routing updates.

Many security schemes have been proposed to enhance the MANET performance. The authors of [59] propose a trust based protocol named AER-AODV, which evaluates direct trust with average encounter rates. An algorithm is proposed in [60] 
that both calculates the route and node trust value. Amaresh et al. [61] introduce a method of jointly considering trust and energy. Jassim et al. [14] present a securityenanced AODV named Reliant Ad hoc on-demand distance vector routing, which allows AODV not only find the shortest paths but also find shortest path that can be trusted. A trust model called forwarding packets is proposed by Li [62] that the model can evaluate the behaviors of neighbors. They present a novel trust mechanism named ad hoc on-demand trusted-path distance vector routing, which is used to discover multiple loop-free path as the trusted path in route discovery. 


\section{Chapter 4}

\section{Trust Based AODV Routing Protocol (TAODV)}

In this chapter, we describe our trust model based on AODV. We assume that if a node in our TAODV protocol broadcasts a packet to its neighbors, the neighbors receive the packet correctly. However, if a node broadcasts multiple packets at the same time, its neighbors only can receive a part of the packets because of some unexpected causes (such as heavy traffic) and malicious attacks (such as black-hole attack and DoS attack). We use a novel concept forwarding ratio and node trust calculation process [62] to evaluate our node trust value.

\subsection{Trust Value Calculation Process}

In this chapter, we describe our trust model. We first introduce the node trust calculation process, and then we illustrate the reverse and forwarding path calculation processes. Thirdly, the objective function of TAODV is described. Finally, we introduce our trust calculation process. 


\subsubsection{Node Trust Calculation Process}

Definition 1(Forwarding ratio): Forwarding ratio is the number of packets received correctly divided by the number of packets forwarded. For example, we assume that a node $a$ sends 120 packets to its neighbor node $b$, and node $b$ only receives 100 packets because of the packet loss. Meanwhile, node $b$ only can forward 80 packets because of its transceiver capability, so the forwarding ratio of node $b$ to node $a$ is 0.8 . The forwarding ratio $R_{a b}(t)$ of node $a$ to node $b$ can be defined by the following formula

$$
R_{a b}(t)=\frac{C_{a b}(t)}{T_{a b}(t)} \quad t \leq W
$$

where $C_{a b}(t)$ represents the number of the packets that a node can correctly forward to its neighbors. $T_{a b}(t)$ denotes the total number of packets that the node received before time $t$, where $W$ represents the width of the recent time window.

In TAODV, the packets can be divided into two groups: control packets (RREQ, RREP and RRER) and data packets [62]. The control packets play a vital role in TAODV protocol. The control packets (RREQ and RREP) determine the data transfer path, and forwarding ratio of control packets is an important factor to determine the node trust value. The node trust computation is shown in below

$$
N_{a b}(t)=\omega_{1} C R_{a b}(t)+\omega_{2} D R_{a b}(t)
$$

where $C R_{a b}(t)$ represents the control packet forwarding ratio and $D R_{a b}(t)$ represents the data packet forwarding ratio. $N_{a b}(t)$ denotes the trust value of receiving node $b$ for forwarding node $a . \omega_{1}$ and $\omega_{2}$ are two weighted factors $\left(\omega_{1}, \omega_{2} \geq 0\right.$, and $\left.\omega_{1}+\omega_{2}=1\right)$ that determine which forwarding ratio $\left(C R_{a b}(t)\right.$ and $\left.D R_{a b}(t)\right)$ is more important in the node trust calculating process. Particularly, we assume $\omega_{1}=1$ and $\omega_{2}=0$, which means that the forwarding ratio of any control packet decides the overall node trust 
value.

\subsubsection{Path Trust Calculation Process}

In the route discovery process, when a control packet such as RREQ arrives at a destination node, the routing path from source to destination is computed according to the node trust defined by the section 4.1.1. According to the axiom [63], concatenation propagation of trust does not increase trust, the reverse and forwarding path trust value should not be more than the trust value of intermediate nodes. Meanwhile, since the control packet is a crucial factor to determine the node trust value, we add a new field called PacketTrust (PT) into the RREQ and RREP packet format and denote by $P T_{\text {rreq }}$ and $P T_{\text {rrep }}$. Specifically, we set the initial value of PacketTrust to 1 . At time $t$, the trust value of a reverse path $P$ is denoted by $T_{P}(t)$ and given by the following formula

$$
T_{p}(t)=N_{a b}(t) \times P T_{a}^{r r e q}
$$

where $P T_{a}^{\text {rreq }}$ means the trust value of PacketTrust field in RREQ packet when a RREQ packet leaves node $a$.

$$
T_{p}^{\prime}(t)=N_{b a}(t) \times P T_{b}^{r r e p}
$$

where $T_{p}^{\prime}(t)$ denotes the trust value of a forwarding path. $P T_{b}^{\text {rrep }}$ means the trust value of PacketTrust field in RREP packet when a RREP packet leaves node $b$. 


\subsubsection{The Objective Function of TAODV}

In our TAODV mechanism, there are two main factors influencing the whole network performance. One factor is hop count, and another is path trust value. Our goal is to evaluate the network performance in three different scenarios: the first one we only consider the path trust factor, the second one we consider both the hop count factor and path trust factor, the third one we only consider the hop count factor. So the objective function of our proposed TAODV protocol is shown in below:

$$
F(x)=\alpha x_{1}+\beta x_{2}
$$

where $F(x)$ denotes the network performance of ad-hoc networks using TAODV protocol. $x_{1}$ denotes the path trust value when control packets arrives at nodes, and $x_{2}$ denotes the hop count of control packets. $x_{1}$ and $x_{2}$ are two influence factors that determine the network performance when using TAODV protocol. $\alpha$ and $\beta(\alpha, \beta \geq 0)$ are two weighted factors.

From this equation, we make three assumptions that help us to analyze the TAODV mechanism:

- When $\alpha \gg \beta$, we assume that the network performance is mainly decided by the path trust value $x_{1}$.

- When $\alpha \approx \beta$, we assume that the network performance is decided by the path trust value $x_{1}$ and hop count $x_{2}$.

- When $\alpha \ll \beta$, we assume that the network performance is decided by the hop count. This scenario is same as the original ad-hoc networks using AODV protocol. 


\subsection{Route Discover Process of TAODV}

The traditional AODV protocol aims to select a minimum hop count path to transfer the data packets. By contrast, in our TAODV protocol, we propose a trust based RREQ (T-RREQ) packet format, which contains the following fields:

(RREQID, HopCount, SourceAddr, SourceSeq, DestAddr, DestSeq, PacketTrust)

As mentioned before, we design a new field named PacketTrust (PT), and add it into a RREQ packet. It is initialized to 1 and varies during the packet transmission process.

In our TAODV protocol, when a node receives a T-RREQ packet from its neighbor, first this node checks the RREQID of this T-RREQ. If the node has already received the same ID before, this T-RREQ is dropped by this node. On the contrary, if the $R R E Q I D$ is new, the routing table of the node checks the sequence number in the RREQ packet, if the sequence number is fresh, the reverse path is established by the node and it updates its own routing table in which calculates the path trust value, if the sequence number is old, the node discards this RREQ packet. Meanwhile, when the node receives the T-RREQ message, it checks its routing table to determine whether this node is destination or have a fresh route to destination. If so, it updates its routing table and responds a T-RREP packet back to the source. If not, the node continues to broadcast this T-RREQ packet to its neighbors. If the node receives the different T-RREQ packets simultaneously, the node chooses a best path in the routing table with better path trust value. In other words, if the new path trust value is better than the previous one, the node updates the routing table immediately. Algorithm 1 depicts the reverse path trust calculation process of TAODV protocol.

Figure 4.5 shows an example of reverse path establishment process of TAODV. We assume that the source node need to initiate the route discovery process. The source 

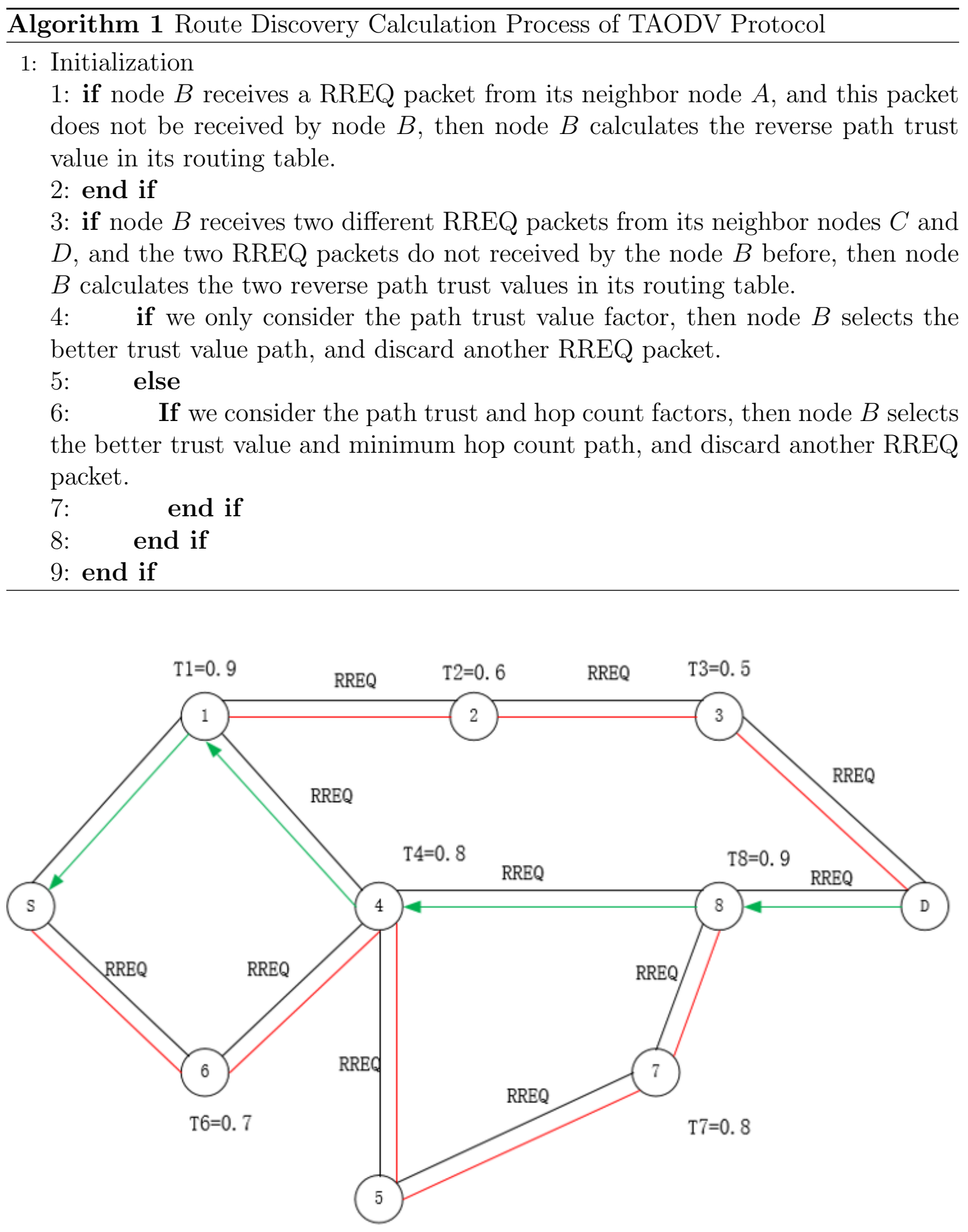

$\mathrm{T} 5=0.6$

Figure 4.1: An example of the TAODV calculation process. 
first broadcasts T-RREQ packets to its neighbor node 1 and node 6. Meanwhile, the PacketTrust field in the T-RREQ is set to 1 . The T-RREQ packets arrive at node 1 and node 6 , the path trust is calculated in (4.3). The path trust value from source to node 1 is $T_{s 1}=0.9 \times 1=0.9$. The path trust from source to node 6 is $T_{s 6}=0.7 \times 1=0.7$. When node 1 and node 6 receive the T-RREQ packets, the value of PacketTrust field of the T-RREQ packets changes to 0.9. Node 4 receives two T-RREQ packets from node 6 and node 1 . The routing table of node 4 compares the path trust value. Here the path trust $T_{64}=0.7 \times 0.8=0.56$ and $T_{14}=0.9 \times 0.8=0.72$. So node 4 discards the T-RREQ packet from node 6 because the path trust value from node 6 to node 1 is smaller than the path trust value from node 1 to node 4 . After the path selection, node 4 sets up the reverse paths to the source. Similarly, the final reverse path is from destination, via node 7 , node 4 , and node 1 to the source.

When receiving a T-RREQ, destination node replies T-RREP back to the source node via the intermediate nodes. Meanwhile, the forwarding paths are established when T-RREP packets pass through the switch nodes. The format of a T-RREP packet contains the following fields:

(SourceAddr, SourceSeq, DestAddr, DestSeq, HopCount, LifeTime, PacketTrust)

After receiving the RREP packet, the source sends the data packets following the forwarding path that established before to the destination node. 


\section{Chapter 5}

\section{Software-defined Mobile Ad-hoc Networks based on TAODV}

In this chapter, we present a novel architecture SD-TAODV for data transmission based on SDN. In the traditional AODV protocol, flow change of transmission packets due to the high node mobility occurs frequently [64]. Control logic and forwarding logic are all concentrated on MANET nodes. By contrast, in our SD-TAODV system, we move the control logic of MANETs from forwarding plane to a control plane in order to improve the network performance.

\subsection{Framework Description}

The framework of SD-TAODV is similar with the traditional SDN architecture. We divide the structure of SD-TAODV into three layers: (1) data forwarding plane operates the TAODV protocol and nodes in the plane support the OpenFlow protocol; (2) NOS (controller) layer aims to manage the network topology and establishes the data transfer path for the data transmission; (3) application layer controls the forwarding rules, routing tables, and routing protocols. The whole SD-TAODV mechanism virtualizes the MANETs and provides the services for the application layer through the 


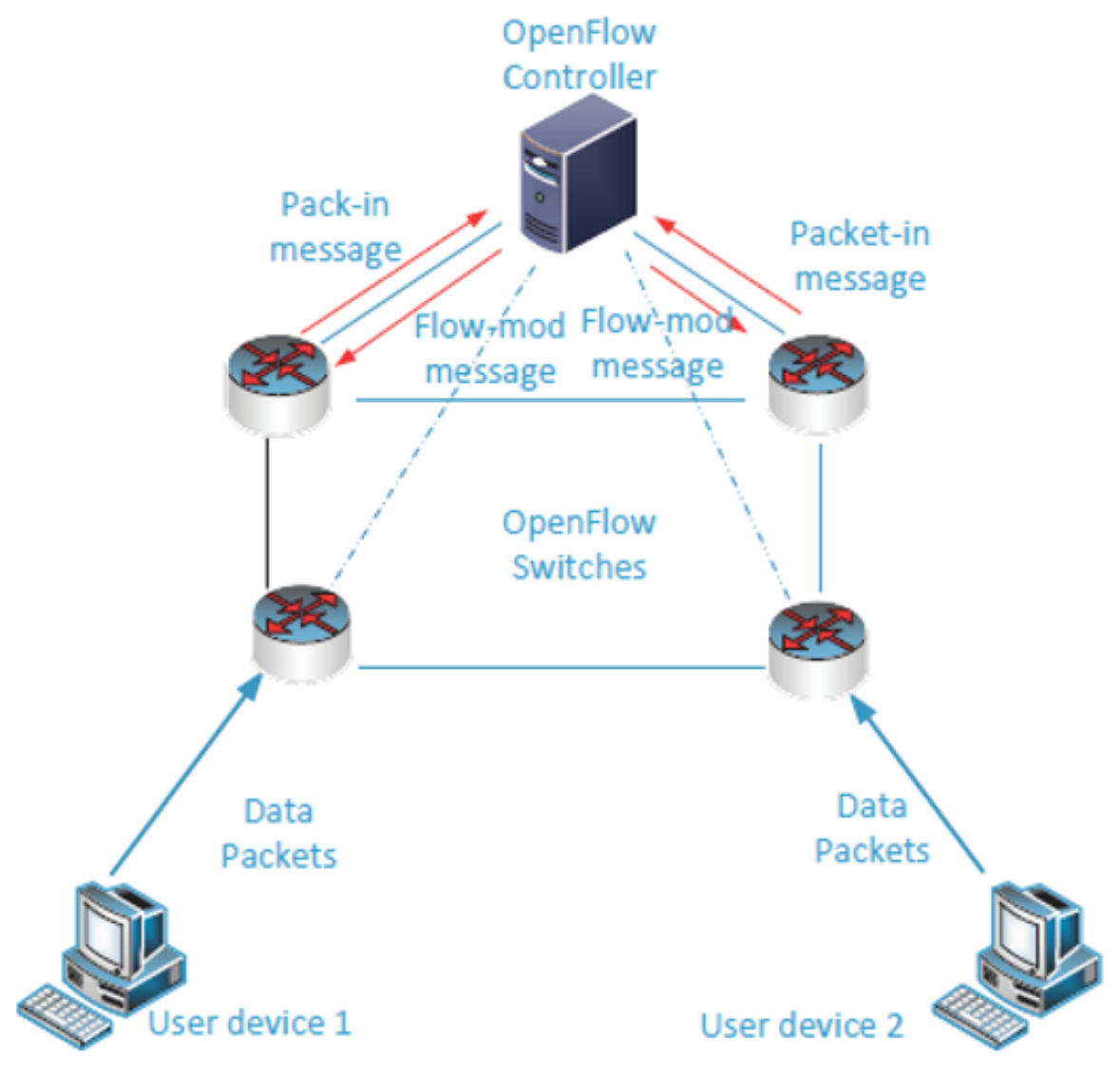

Figure 5.1: Traditional OpenFlow switches and controller interaction using OpenFlow messages.

OpenFlow interfaces.

Figure 5.1 shows the data transfer process of the traditional SDN architecture. For the original OpenFlow structure, no matter the switches or the controller, they are all fixed. However, the TAODV topology always keep changing because of the node mobility, so the architecture of SD-TAODV should be different from the traditional SDN. As shown in Figure 5.1, the information interaction of the traditional OpenFlow has three types: user devices and OpenFlow switches interaction, switches and OpenFlow controller interaction, and switches' interaction. In the SD-TAODV, as shown in Figure 5.2, node 1 acts as a controller that manages the whole network. 


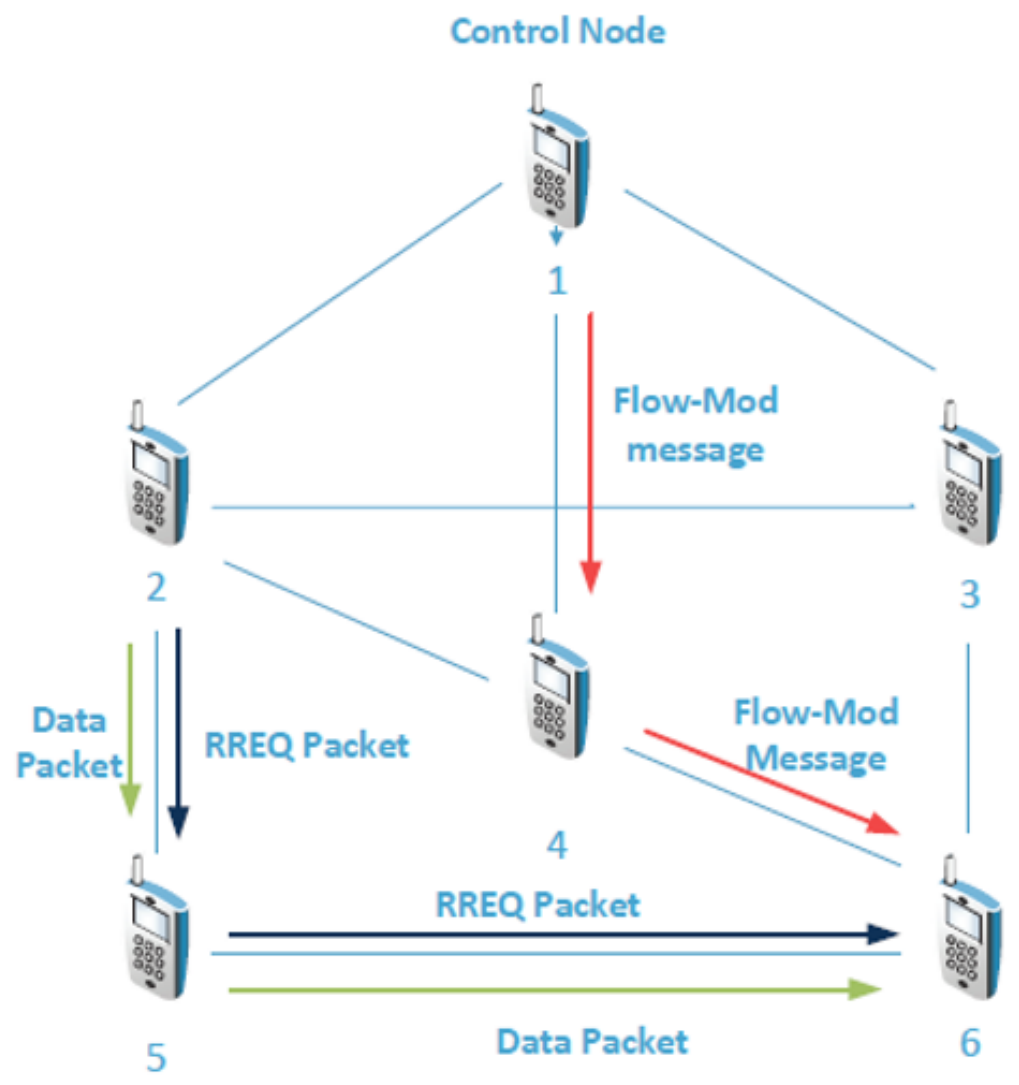

Figure 5.2: An example Mobile ad-hoc network using SD-TAODV mechanism.

There are three different types of information interactions in the SD-TAODV network: controller-to-switch information interaction, control packets interaction, and data packets interaction. For example, controller-to-switch includes control node 1 sends OpenFlow messages to the forwarding nodes (Flow-mod message), and the control packets interaction includes node 2 sends control packets (RREQ or RREP) to node 6 .

Figure 5.3 depicts the working process of switches. Briefly, if a switch node receives a TAODV control packet (T-RREQ or T-RREP), it sends the packet to the controller to handle. If a switch node receives a data packet, it forwards the packet to its neighbor node(s). The centralized control mechanism in SD-TAODV manages the whole network in the control node. So the control node first needs to know the whole 


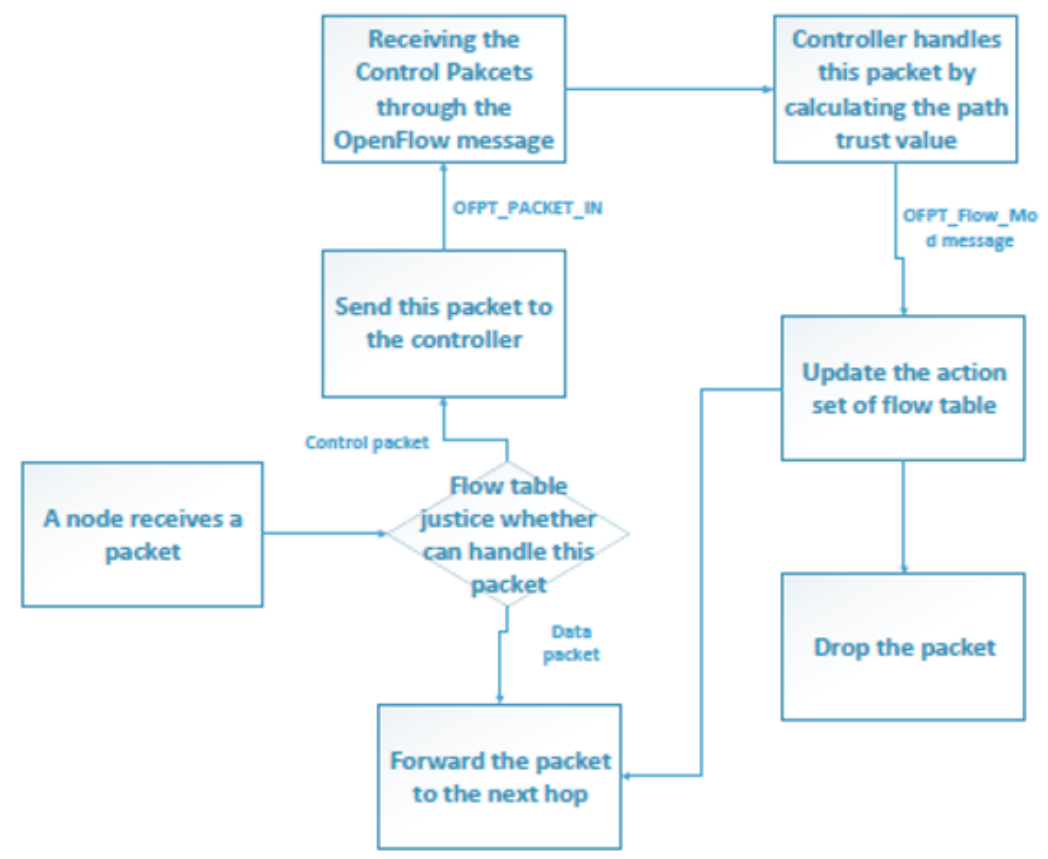

Figure 5.3: The working process of switches based on TAODV protocol.

network topology.

SD-TAODV intensively control and manage the entire network through developing the routing strategies, so the control node must have a whole network topology information. So we need to design a message to obtain the entire network topology in NOS layer. The message can be designed by broadcasting node information to the entire network, and then control node can get the topology of the entire network.

In SD-TAODV, if choosing a fixed node as the control node, because of the node mobility, we cannot exclude a situation that the node maybe leaves the network at any time. Therefore, this situation brings a key issue that how to choose a terminal node as a controller for the whole network. One solution is that try to run a control program on each node. Although such programs are relatively simple, they also can bring some problems. For example, the control programs in two neighbor nodes may clash with each other, and how to choose one of the nodes (each node running the 
control program) as the controller; another solution is that automatically assigns the best status network node as the control node. The best status node means that the node has the highest forwarding ratio. We can factitiously select the best status node to serve as the controller. Finally, setting a standby control node is necessary because the standby node can quickly restore communication network if the precious control node is lost.

\subsection{The Control and Forwarding Logic Separation Method}

As we described before, the separation method is to design a mechanism that makes the controller get the whole network topology. The method of discovering the network topology is that the control node sends topology request message to its neighbor(s). The topology request message includes the following fields:

(PacketID, ControllerAddr, NodeTrustList, TopologyList)

The NodeTrustList is used to record the trust value of nodes when a topology request message has passed by. When the message arrives at any one of the nodes in the network topology, the TopologyList records this node information and adds the information into the TopologyList field. Specifically, if a node receives the same PacketID request message, the node sends back this packet to the controller immediately. Similarly, one of nodes in the network topology sends the massage back to the controller if all of its neighbor nodes have already in the TopologyList field. When the control node receives all the responses, the controller can establish the whole network topology or updates the exciting topology. Figure 5.2 shows an example of the TAODV network topology. The method of control node 1 to get the whole network topology is shown in below: 
1) Controller 1 initializes the topology request messages (e.g ID is 1357), and adds them into the TopologyList. Controller 1 sends the request messages to its neighbors, nodes 2 and 3 .

2) Node 2 receives the request packet and adds itself into the TopologyList. Meanwhile, node 2 puts its own trust value into the NodeTrustList, and then node 2 broadcasts the message to its neighbor, since the TopologyList already has the information of node 1 , so node 2 only transfers the message to nodes 3,4 and 5. Similarly, node 3 only sends the request packet to its neighbor 6 . Meanwhile, node 3 sends the message back to node 2 because the PacketID is the same. Node 4 receives the request message from the node 2 , and node 4 puts itself into the TopologyList. Since node 2 already exists in the TopologyList, node 4 only transfers the message to the node 6 . Analogously, node 5 also sends the request message to node 6 .

3) Node 6 receives the request messages from nodes 3, 4 and 5. First node 6 puts its routing information into the TopologyList, and adds trust information into the NodeTrustList. Secondly, since the PacketID of three packets is the same, so node 6 sends back these three messages back to the controller 1 according to the Controller Addr.

4) When receiving all the responses from other nodes, controller 1 enables to get the whole network topology.

\subsection{The Design Method of Controller}

The SD-TAODV control node is responsible for the entire network management and control, and the forwarding node supports OpenFlow protocol. OpenFlow protocol used to send messages and resolve. 


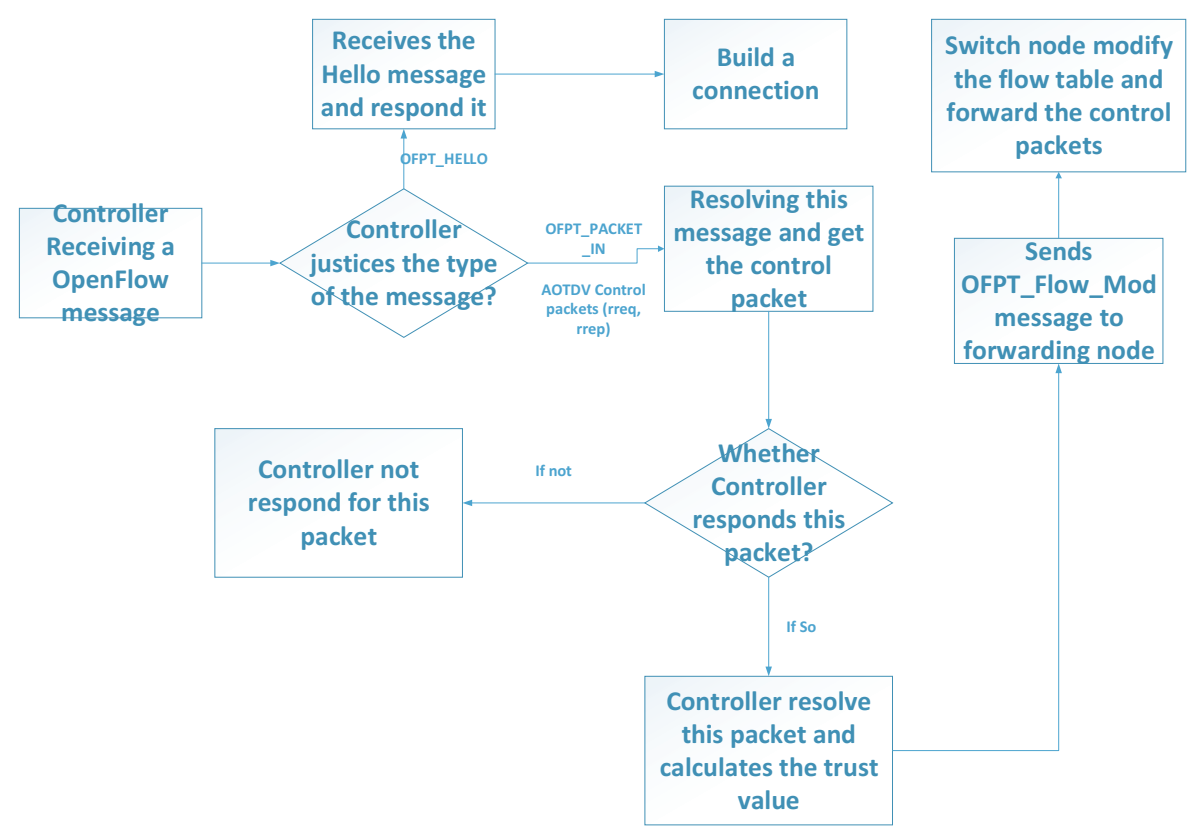

Figure 5.4: The working process of control node based on TAODV protocol.

\subsubsection{The Working Process of the Controller}

After getting the network topology, the controller can control and manage the whole network. The information interaction method between the control node and the OpenFlow switches includes the OpenFlow messages such as OFPTFlowMod, OF PT PacketIn and OFPT Hello. The interfaces between the controller and OpenFlow switches are similar to the traditional southbound API.

The working process of the control node is shown in Figure 5.4. When receiving an OpenFlow message from a forwarding node, the controller determines the type of the message, if the message is OFPTHello, the controller responds the message and builds a connection between the node and controller. If the message is the OFPTPacketIn, the control node resolves the message and gets the message information, which includes the details of the T-RREQ and T-RREP packet. As we described before, when a T-RREQ or T-RREP packet arrives at the control node, 
the controller gets the value in PacketTrust field, and calculates the path trust value. After finishing the packets handling, the controller sends an OFPTFlowMod message to the forwarding node.

\subsubsection{The Implementation of OpenFlow Protocol in SD- TAODV}

As previously described, the SD-TAODV control node and the forwarding node configuration all support OpenFlow protocol. In order to implement OpenFlow protocol in SD-TAODV network, the underlying MANET network layer needs to have appropriate interfaces supported for southbound API.

The MANET network layer is used to transmit and analyze the messages, including OFPTHELLO, OFPTPACKETIN, and OFPTFLOWMOD.

All OpenFlow messages are derived from the same abstract base class OFMessage. For example, OFPTHELLO message is used to build the connection between the control node and forwarding node. The message contains only the head of protocol. The variables of the OFPTHELLO message include: version, type, length, and transaction. The version represents the protocol version number; type depicts the types of the protocol message; length represents the total length of the protocol message; transaction is used to report a unique number of the OFPTHELLO message.

OFPTPACKETIN message for forwarding node is used to request a flow table. The main parts of the OFPTPACKETIN include: bufferID, totallength, inportNo, OFPacketReason, and PacketData. Specifically, the OFPacketReason field represents the reason why switch nodes request the flow table. The functionality of PacketData field is used to forward data. 


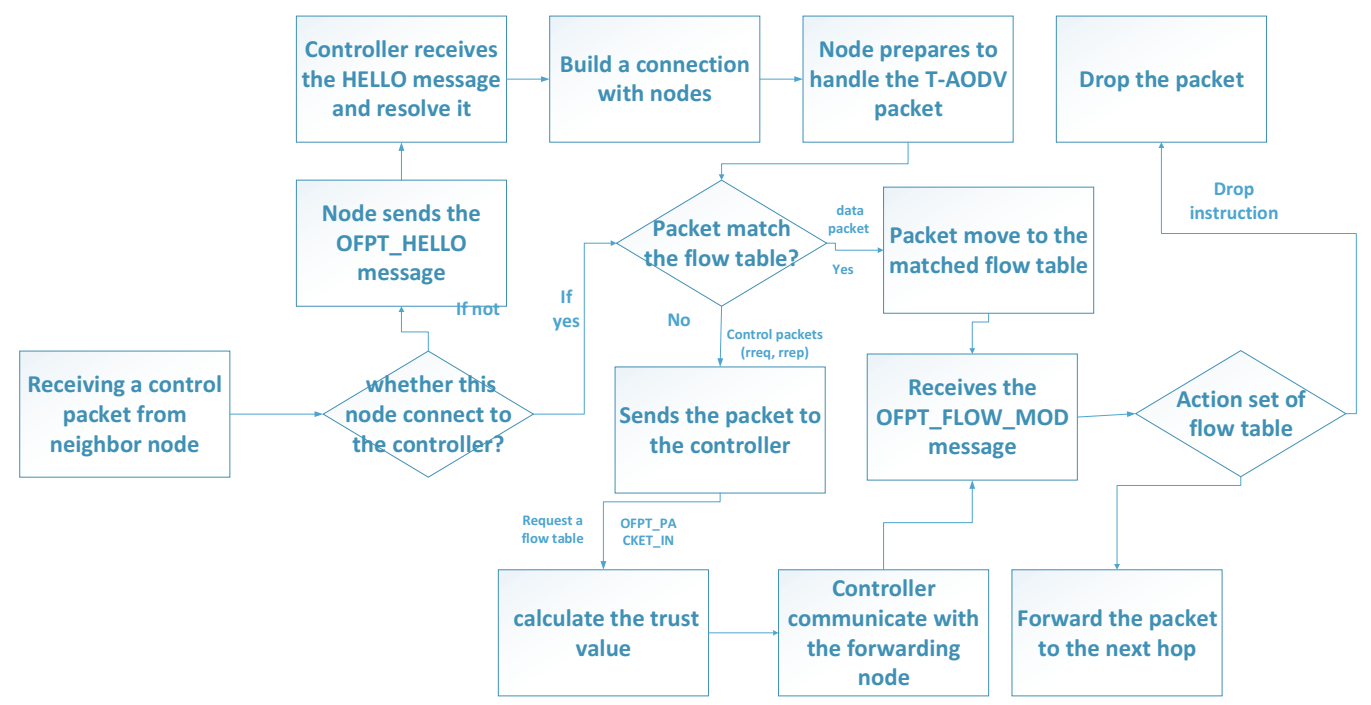

Figure 5.5: The working process of forwarding node based on TAODV protocol.

The most critical one is OFPTFLOWMOD message, which includes the following main variables: MatchField, Cookie, command, idleTimeout, hardTimeout, Priority, bufferID, and Flags.

All OpenFlow protocol messages are encapsulated in the UDP packets and transmitted to other nodes. The forwarding nodes establish the UDP connection with the control node by monitoring the control node, and then send the encapsulated OpenFlow protocol message to the control node, the control node resolves the message and responds to the forwarding nodes. After the forwarding nodes receive the responses, they follow the instructions to execute the actions.

\subsection{The Design of Forwarding Nodes}

The forwarding nodes in the SD-TAODV are used to transfer the data packets and control packets. The interfaces between the control node and switches are the southbound API, which supports the OFPT Hello, OF PT FlowMod and OF PT PacketIn messages. 
In the SD-TAODV system, the forwarding nodes send the OF PT Hello messages to the control node periodically. If any node in the network topology receives the response from the control node, this forwarding node builds a connection with the control node. If a forwarding node receives a control packet such as T-RREQ packet from its neighbors, the T-RREQ packet first matches the flow table (we assume that RREQ cannot match the flow table). Otherwise, the forwarding node sends this packet to the controller in order to request a new flow table with the OF PT PacketIn message. After resolving the packet, the control node responds an OFPTFlowMod message back to the node and modifies the flow table, and executes the action set in the flow table to handle this packet. Figure 5.5 describes the working process of the forwarding node. 


\section{Chapter 6}

\section{Simulation Results and Discussions}

In this chapter, we describe our simulation setup, configuration, and simulation results. OPNET is used as the simulator. In SD-TAODV simulations, we consider two different scenarios in the route discover process: the first one only considers the path trust value factor, and the second one both considers the hop count factor and path trust value. Moreover, in our simulations, we assume that the nodes in our TAODV is all SDN-enabled. In addition, we assume that our SD-TAODV network includes two different types of nodes: i) normal nodes, which the data packets are normally forwarded by those nodes; ii) malicious nodes, which randomly drop the data packets when they receive the packets. The number of the malicious nodes is much smaller compared with the number of normal nodes.

\subsection{SD-TAODV Simulation Setup}

Our simulation model is built on the OPNET Modeler. We consider three different situations that we described in Chapter 4. In our simulation, We choose the highest forwarding ratio node as the controller, and reverse and forwarding path trust value is calculated by the (4.3) and (4.4). The simulation parameters are as follows:

1) Packet size: 1024 bytes 
2) Transport protocol: UDP

3) Network protocol: OpenFlow

4) Routing Protocol: AODV

5) MAC protocol: IEEE 802.11

6) Physical protocol: IEEE $802.11 \mathrm{~b}$

7) Data rate: 1 Mbps, 2 Mbps, 5.5 Mbps and 11 Mbps

8) Simulation area: $5 \times 5 \mathrm{~km}^{2}$

9) Number of nodes: 10, 13, 16, 19, 22, 25

10) Simulation time: $15 \mathrm{mins}$

There are four metrics evaluated in our simulation:

- Average end-to-end delay: the average end-to-end delay is the time that is calculated by the data packets to be transferred across the whole network from the source node to the destination node. It includes buffer delays during the route discover process, queuing delays at interface queues, retransmission delays at MAC layers, and the propagation time from the source to the destination [62].

- Network throughput: the throughput is the total size of packets received by the destination node at every second. The network throughput is an important factor that evaluates the network performance.

- Total message sent: the total message sent is amount of the routing traffic sent in the entire network.

- Packet delivery ratio (PDR): PDR is the ratio of the number of data packets received by a destination node and the number of the data packets generated by a source node. 


\subsection{Evaluation}

Firstly, the average end-to-end delay of the proposed SD-TAODV scheme is evaluated through Figure 6.1 and Figure 6.2. From these two figures, we can see that the endto-end delay of the SD-TAODV is higher than the traditional AODV with different data rates (1 Mbps, 2 Mbps, 5.5 Mbps and $11 \mathrm{Mbps}$ ). Similarly, Figure 6.3 and Figure 6.4 depict the comparison of the end-to-end delay with the time variation, in which the average end-to-end delay of the SD-TAODV system is still higher than the traditional AODV protocol. The reason why the end-to-end delay increasing as the data rates grow is that the quality of channels becomes more and more bad as the node velocities grow, so the risk probability of packet loss in the channels increases. As the data rates grow, the packets are easier to be dropped in the channels, so the end-toend increasing as the data rates grow. There are two reasons for the higher end-to-end delay of SD-TAODV: i) in the route discovery process, the SD-TAODV nodes always select fresh and higher path trust value routes to establish reverse and forwarding paths in order to transfer the data packets to the destination. The best trust value route reduces the risk probability of route breakdown because of the drop. However, the new routes may have more hop counts to the destination than the traditional AODV. The data packets need to spend more time to be transferred in the new routes; and ii) for the SD-TAODV scheme, the nodes first need to build connection with the control node. When TAODV packets (T-RREQ, T-RREP) arrive, nodes send the control packets to the controller to handle. This process also needs to spend some time. So comparing with the traditional AODV network, the end-to-end delay of SD-TAODV is higher.

Figure 6.5 depicts the throughput comparison of the SD-TAODV and traditional AODV in different data rates. Through Figure 6.5, we can conclude that the performance of the proposed SD-TAODV mechanism is better than the traditional 


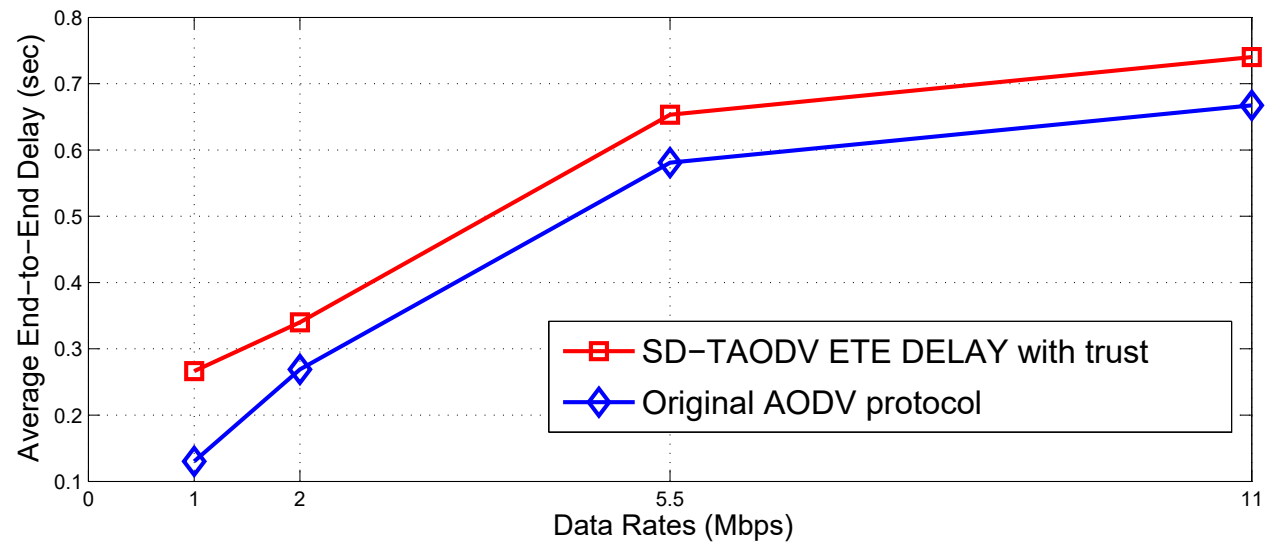

Figure 6.1: The average ETE delay of SD-TAODV with trust value in different data rates.

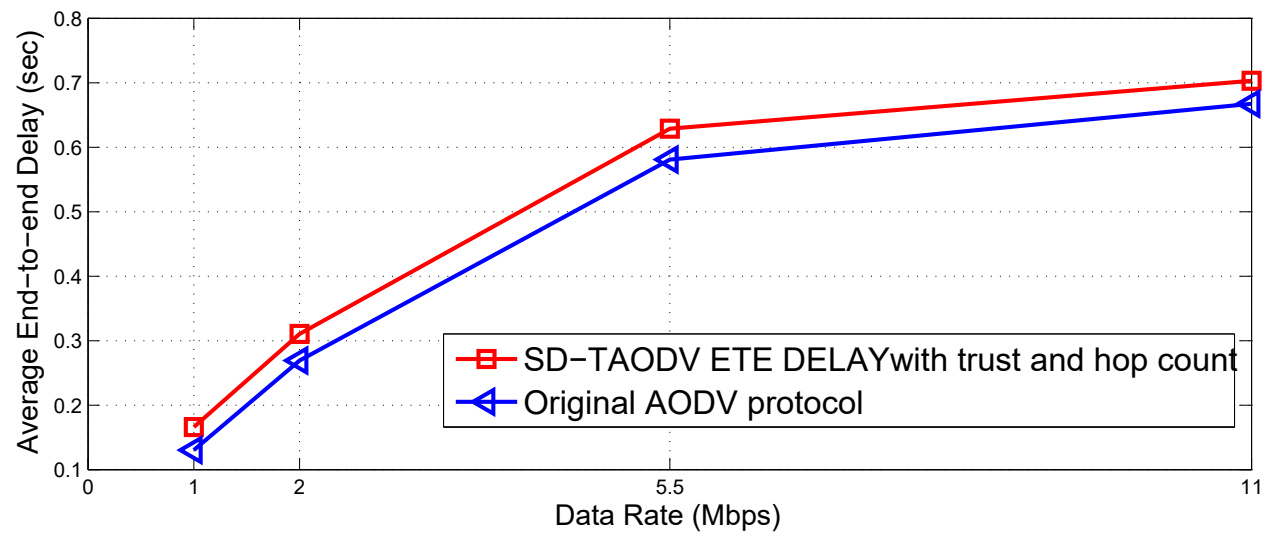

Figure 6.2: The average ETE delay of SD-TAODV with trust value and hop count in different data rates. 


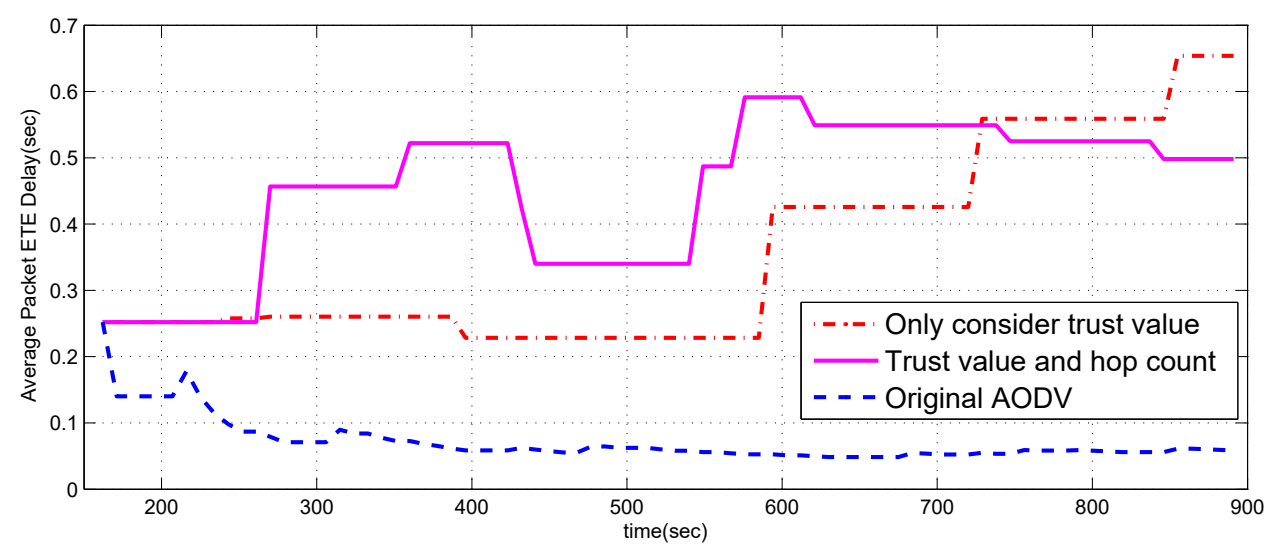

Figure 6.3: The average ETE delay comparison of SD-TAODV with the time variation in 5.5 Mbps.

AODV protocol. Specifically, we also compare the network throughput in the case of the $5.5 \mathrm{Mbps}$ and $11 \mathrm{Mbps}$ within the time variation. Figure 6.6 and Figure 6.7 show the network throughput of the SD-TAODV with trust and hop count and the SD-TAODV only with the trust value are both better than the traditional AODV. These two scenarios indicate that the network performance of the SD-TAODV is also better than the traditional AODV network. As the data rates grow, we can see that the throughput of both the SD-TAODV and original AODV all increases. This is because more data packets can be received as the data rates grow. The reason for the better performance of the SD-TAODV is that the best trust value path is selected by the SD-TAODV system, which means that the selected path between two nodes reduces the risk of packet loss and the quality of links are better than the traditional AODV. In other word, as the data packets are transferred on the secure paths, the possibilities of packets loss are lower than the traditional AODV. So the SD-TAODV scheme has performance improvement in terms of network throughput compared to traditional AODV.

In addtion, we also evaluate the SD-TAODV network performance in different numbers of MANET nodes. In Figure 6.8, we can see that the throughput of the 


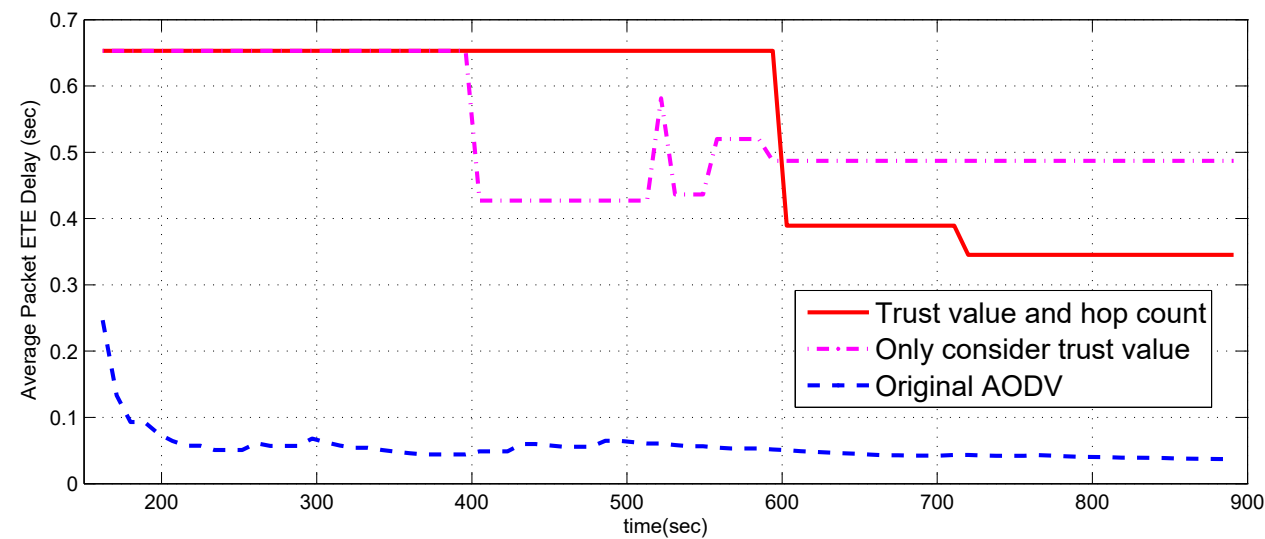

Figure 6.4: The average ETE delay comparison of SD-TAODV with the time variation in 11 Mbps.

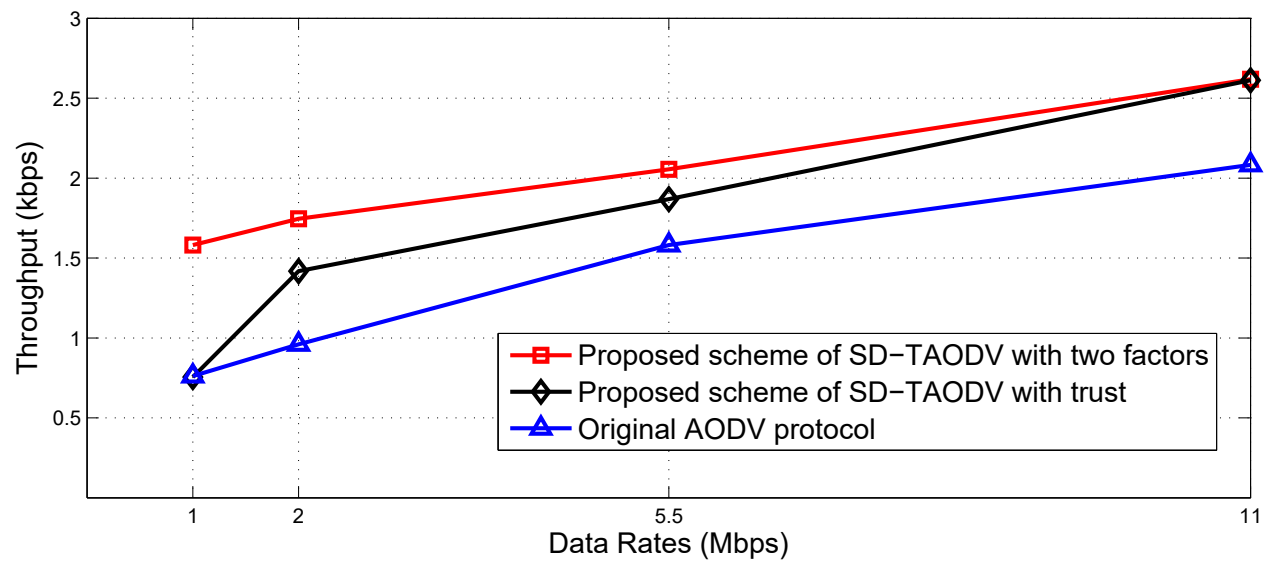

Figure 6.5: The average throughput comparison of SD-TAODV with different data rates. 


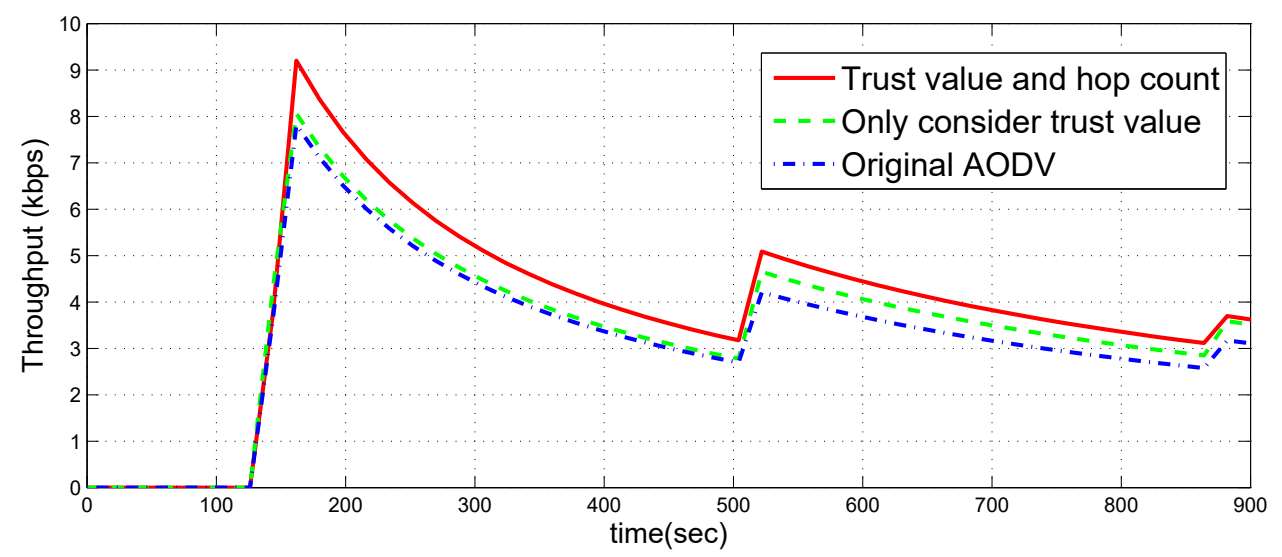

Figure 6.6: The average throughput comparison of SD-TAODV with time variation in $5.5 \mathrm{Mbps}$.

SD-TAODV and original AODV all decreases as the number of nodes grows. We assume that the number of malicious nodes increases as the number of nodes grows. The malicious nodes also have big impact on the throughput of the SD-TAODV network [65]. The network throughput decreases significantly, as shown in Figure 6.8. Although the network throughput decreases as the number of nodes grows, the SD-TAODV network throughput is still better than the original scheme. Because network nodes update their neighbors' information periodically, the control node of SD-TAODV can respond faster to the topology change. As the network nodes leave or join the network, the control node detects the topology change and sends the control message to these new nodes to maintain the data transfer path, which means that the possibilities of links interruption decrease. So our proposed scheme has performance improvement than the traditional AODV.

Meanwhile, we compare the total message overhead sent in different nodes. Figure 6.9 depicts how much message overhead sent by the SD-TAODV mechanism compared with the traditional AODV protocol. Through Figure 6.9, we can conclude that the message overhead of the SD-TAODV is higher than the original AODV. This is because the nodes in the SD-TAODV network need to send extraneous messages such 


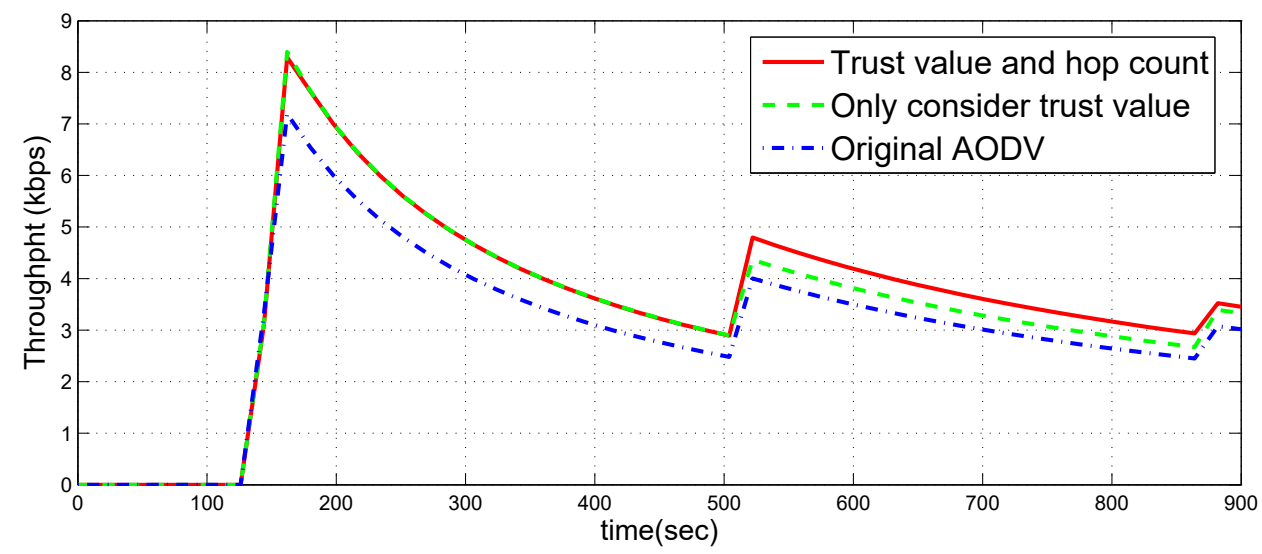

Figure 6.7: The average throughput comparison of SD-TAODV with time variation in 11 Mbps.

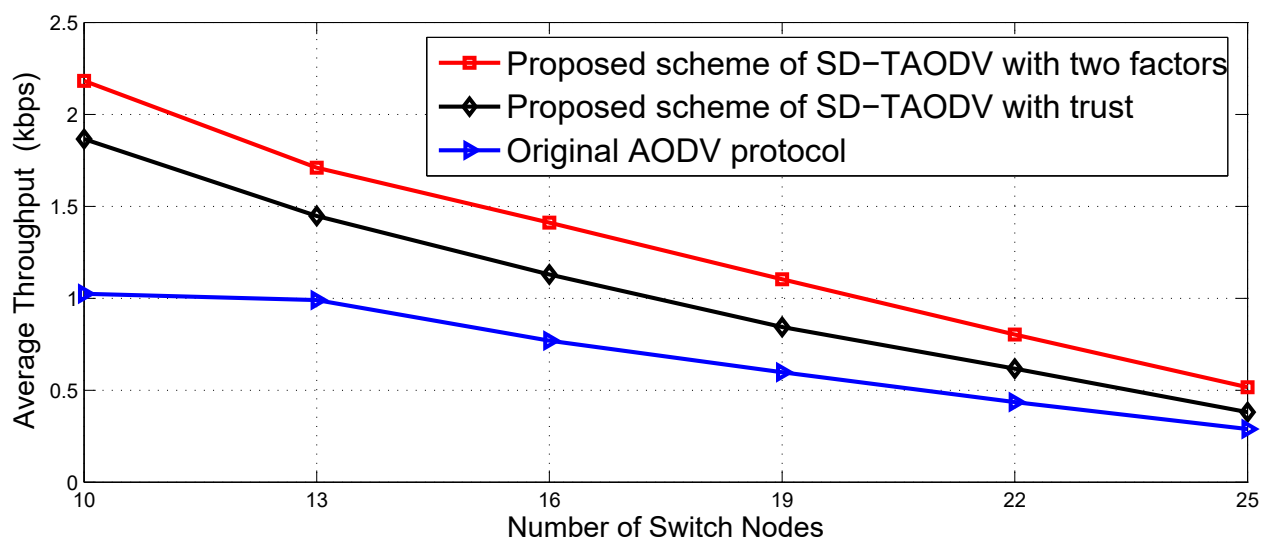

Figure 6.8: The average throughput comparison of SD-TAODV number of nodes.

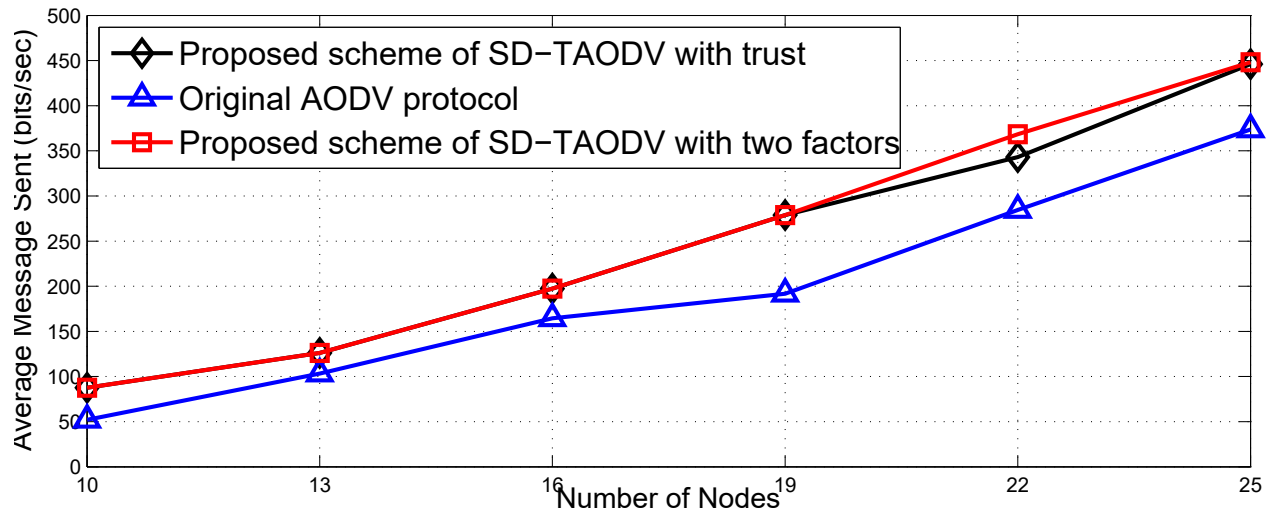

Figure 6.9: Total message overhead comparison in different number of nodes. 


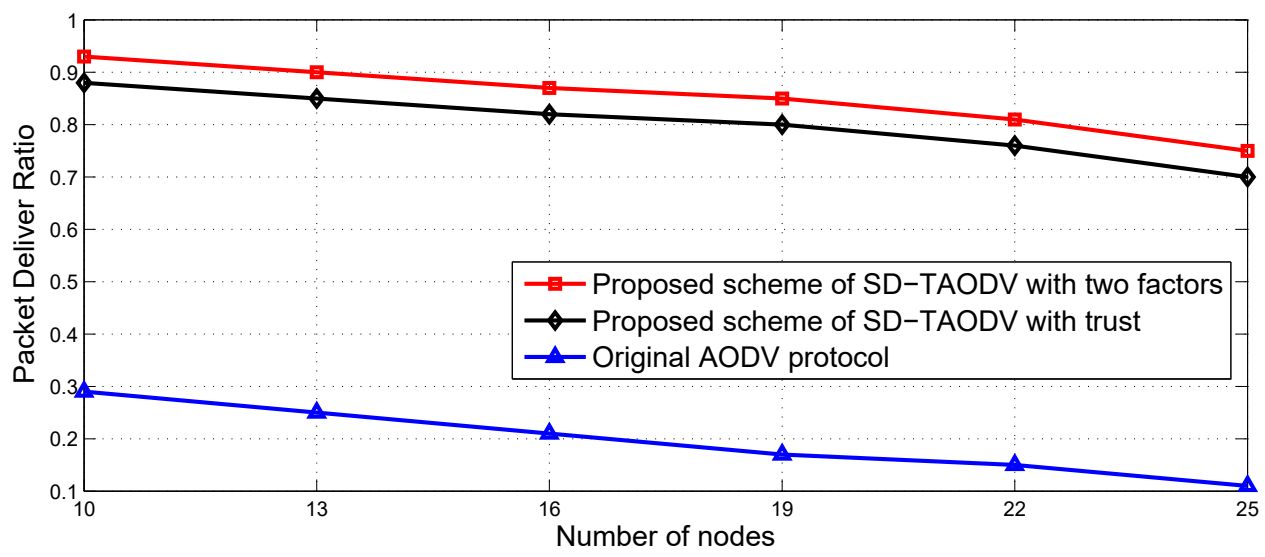

Figure 6.10: PDR versus the number of nodes.

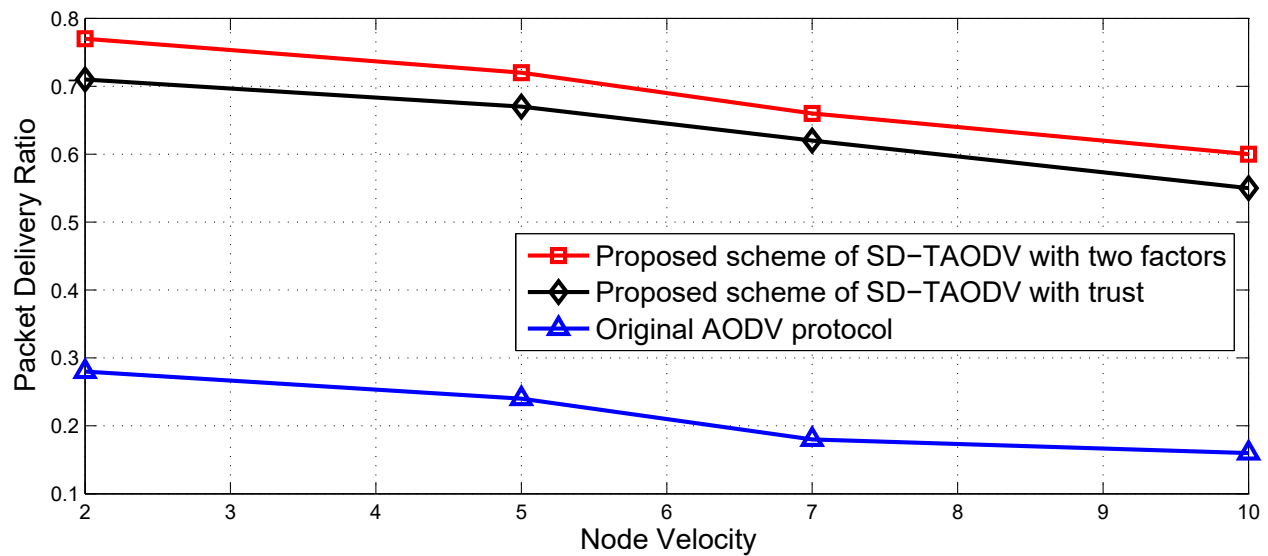

Figure 6.11: PDR versus the node velocity. 
as OFPT Hello and OFPT PacketIn to the control node. In Figure 6.9, since more nodes join the network as the number of node increases, so the message overhead grows simultaneously in both AODV and the SD-TAODV system.

In Figure 6.10, we also compare PDR with different number of nodes. From the Figure 6.10, we can conclude that the PDR of our proposed scheme is better than the traditional AODV scheme. there are two reasons for the higher PDR of the proposed SD-TAODV scheme: i) the reverse and forwarding path trust calculation of our proposed SD-TAODV can detect and reduce the misbehavior of malicious nodes, and ii) the SD-TAODV scheme reduces the possibilities of interruptions of the routing paths. The results also illustrate that the proposed scheme with the hop count factor and path trust value factor has the highest PDR among the three schemes. Through this figure, we also can demonstrate that the PDR of the three schemes are all decrease as the number of nodes grow. This is because that the number of the malicious nodes increases simultaneously as the number of nodes grows, so the misbehavior of the malicious nodes increase the possibilities of packet loss in the three schemes.

Finally, we compare the PDR with different node velocities. In Figure 6.11, as the node velocity increases, the PDR of all three schemes decreases significantly. This is because that the quality of channels becomes more and more bad as the node velocity increases, so the packets are easier to be dropped in the transmission. However, the PDR of the proposed SD-TAODV scheme is still better than the original AODV protocol. 


\section{Chapter 7}

\section{Conclusions and Future Works}

In this thesis, we mainly focused on the trust based MANET routing protocols and SDN technology. We discussed the development, architecture, routing protocols and the problems of MANETs. Meanwhile, We introduced SDN frame and analyzed the main characteristics of SDN, OpenFlow, and its southbound and northbound interfaces. We have presented a novel framework of software-defined MANETs with trust management. We designed a routing protocol named software-defined trust based ad hoc on-demand distance vector routing (SD-TAODV). In the SD-TAODV scheme, the route discovery and the route maintenance process are moved into a controller, and the reverse and forwarding paths are chosen by the controller. Simulation results were presented to show the effectiveness of the proposed software-defined MANETs with trust management. We compared our SD-TAODV protocol with the traditional AODV protocol in terms of end-to-end delay, throughput and message overhead. Although the end-to-end delay and the message overhead of SD-TAODV are higher than AODV, the network throughput performance improves significantly in our proposed SD-TAODV mechanism. The main research works are concluded as follows:

- We summarized MANET concept, development, features, applications and key technologies. On the basis of the current researches, we introduced the ad hoc 
network routing protocols. We discussed the existing ad hoc network routing protocol classification method that includes the Proactive routing protocols and reactive routing protocols. We have introduced some examples of these two kinds of routing protocols. We summarized the problems of current ad hoc networks, and concluded the current researches about the trust based MANET routing protocols.

- We simply introduced the concepts and features of SDN and OpenFlow. We have analyzed the OpenFlow architecture, matched process of flow table, and information interaction of OpenFlow. All these features can be used in our proposed SD-TAODV mechanism.

- We have modified the original AODV protocol that adds trust mechanism into our trust model. We referred a forwarding ratio concept to establish our trust model. First we have defined the node trust value and path trust value, and then we described our objective function of TAODV. We considered different situations to evaluate our proposed mechanism.

- We also proposed a new AODV network frame (SD-TAODV). We have combined the SDN concept with the AODV protocol. In SD-TAODV, the route discovery and the route maintenance process are all moved into the control node, in which the reverse and forwarding paths are chose by the control node. Meanwhile, the route maintenance such as local repair is also decided by the controller. In our simulation, each node is given a node trust value based on the forwarding ratio concepts. We compared our SD-TAODV model with traditional AODV in end-to-end delay, throughput and message overhead. Although the end-to-end delay and the message overhead of SD-TAODV are higher than the AODV protocol, the network performance improves significantly in SD-TAODV 
system.

In the future, we will aim to reduce the end-to-end delay of our proposed scheme. Meanwhile, in our SD-TAODV, since our controller is a moving node, we wish to design a mechanism that can choose a new control node if the previous controller is lost. 


\section{List of References}

[1] M. Yadav and N. Uparosiya, "Survey on MANET: routing protocols, advantages, problems and security," Int. J. Innovative Computer Science and Engineering, vol. 1, no. 2, pp. 12-17, 2014.

[2] N. M. Mosharaf, K. Chowdhury, and R. Boutaba, "A survey of network virtualization," Computer Networks, vol. 54, pp. 862-876, 2009.

[3] H. Kim and N. Feamster, "Improving network management with software defined networkinging," IEEE Comm. Mag., vol. 51, no. 2, pp. 114-119, 2013.

[4] S. Sezer, S. Soctt-Hayward, P. K. Chouhan, B. Fraser, D. Lake, J. Finnegan, N. Viljoen, M. Miller, and N. Rao, "Are we ready for SDN? implementation challenges for software-defined networks," IEEE Comm. Mag., vol. 51, no. 7, pp. 36-43, 2013.

[5] K. Kirkpatrick, "Software-defined networking," Communication of The ACM, vol. 56, no. 9, pp. 16-19, 2013.

[6] N. Feamster, J. Rexford, and E. Zegura, "The road to SDN: an intellectual history of programmable networks," ACM SIGCOMM Computer Communication Reivew (CCR), vol. 44, no. 2, pp. 87-98, 2014.

[7] W. Xia, Y. Wen, C. Foh, D. Niyato, and H. Xie, "A survey on software defined networking," IEEE Commun. Surveys \& Tutorials, vol. 17, no. 1, pp. 27-51, 2015 .

[8] D. Kreutz, M. Ramos, P. Verissimo, C. Rothenberg, S. Azodolmolky, and S. Uhlig, "Software-defined networking: A comprehensive survey," Proc. IEEE, vol. 103, no. 1, pp. 14-76, 2015.

[9] D. N. Patel, S. B. Patel, H. R. Kothadiya, P. D. Jethwa, and R. H. Jhaveri, "A survey of reactive routing protocols of MANETs," in Proc. IEEE Int. Conf. 
Information Communication and Embedded System (ICICES), (Chennai), pp. 16, 2014.

[10] S. Mo, J. Gu, R. Ghanadan, M. Sherman, J. Farkas, J. Tranquilli, J. Niedzwiecki, and B. Fette, "Distributed scheduler design for multiuser detection enabled wireless mobile ad-hoc networks," in Proc. Int. Conf. Military Communications (MILCOM), (San Jose, CA), pp. 98-103, 2010.

[11] Q. Chen, U. Ozguner, and K. Redmill, "Ohio state university at the 2004 DARPA grand challenge: developing a completely autonomous vehicle," IEEE Intelligent Systems, vol. 19, no. 5, pp. 8-11, 2004.

[12] "The mobile computing and multimedia laboratory university of maryland, college park." Website. http://www.cs.umd.edu/projects/mcml/.

[13] K. Chawda and D. Gorana, "A survey of energy efficient routing protocol in MANETs," in Proc. IEEE Int. Conf. Electronics and Communication System (ICECS), (Coimbatore), pp. 953-957, 2015.

[14] H. Jassim, S. Yussof, S. K. T. Kiong, and R. Ismail, "A routing protocol based on trusted shortest path selection for mobile ad hoc network," in Proc. IEEE ICC, (Kuala Lumpur), pp. 547-554, 2009.

[15] G. Tomar, T. Sharma, D. Bhattacharyya, , and T. Kim, "Performance comparison of AODV, DSR and DSDV under various network conditions: A survey," in Proc. IEEE Int. Conf. Ubiquitous Computing and Multimedia Applications (UCMA), (Daejeon), pp. 3-7, 2011.

[16] C. Perkins, E. Belding-Royer, and S. Das, "Ad hoc on-demand distance vector (AODV) routing," tech. rep., RFC 3561, Jul. 2003.

[17] Y. Chen, Y. Shen, X. Jiang, and J. Li, "Throughput capacity of ALOHA MANETs," in Proc. IEEE Int. Conf. Communication in Cina (CIC), (Xi'an, China), pp. 71-75, 2013.

[18] D. A. Beyer, "Accomplishments of the DARPA SURAN program," in Proc. Int. Conf. Military Communications (MILCOM), (Monterey, CA), pp. 855-862, 1990.

[19] R. Alturki and R. Mehmood, "Multimedia ad hoc networks: performance analysis," in Proc. IEEE Int. Symp. Computer Simulation and Modeling EMS'08, (Liverpool, UK), pp. 561-566, 2008. 
[20] M. Frofigh, P. Johansson, and P. Larsson, "Wireless ad hoc networking-the art of networking without a network," Ericsson Review, no. 04, pp. 248-263, 2000.

[21] D. Djenouri, L. Khelladi, and A. N. Badache, "A survey of security issues in mobile ad hoc and sensor networks," IEEE Commun. Surveys $\&$ Tutorials, vol. 7 , no. 4, pp. 2-28, 2006.

[22] M. Casado, M. J. Freedman, J. Pettit, N. G. J. Luo, N. Mckeown, and S. Shenker, "Rethinking enterprise network control," ACM/IEEE Trans. Networking, vol. 17, no. 4, pp. 1270-1283, 2009.

[23] "Openflow switch specification." Website, Sep. 2012. https://www.opennetworking.org/images/stories/downloads/sdn-resources/onfspecifications/openflow/openflow-spec-v1.3.1.pdf.

[24] N. Mckeown, T. Anderson, H. Balakrishanan, G. Parulkar, L. Peterson, J. Rexford, S. Shenker, and J. Turner, "Openflow: enabling innovation in campus networks," ACM SIGCOMM Computer Communication Reivew (CCR), vol. 38, no. 2, pp. 69-74, 2008.

[25] Z. Ismail and R. Hassan, "Effects of packet size on AODV routing protocol implementation in homogeneous and heterogeneous MANETs," in Proc. IEEE Int. Conf. Computer Intelligence, Modeling and Simulation, (Langkawi, Malaysia), pp. 351-356, 2011.

[26] A. Boukerche, "Performance comparison and analysis of ad hoc routing algorithms," in Proc. IEEE Int. Conf. Performance, Computing and Communications, (Phoenix, AZ), pp. 171-178, 2011.

[27] H. Yang, H. Luo, F. Ye, S. Lu, and L. Zhang, "Security in mobile ad hoc netowks: challenges and solutions," IEEE Trans. Wireless Commun., vol. 11, no. 1, pp. 3847, 2004.

[28] D. Loganathan and P. Ramamoorthy, "Efficient routing with multicast parameters based DSDV protocol in wireless ad hoc networks," in Proc. IEEE Int. Conf. Pattern Recognition, Informatics and Mobile Engineering (PRIME), (Salem, Massachusetts), pp. 435-439, 2013.

[29] K. Palani and P. Ramamoorthy, "Performance evaluation of QoS based DSDV protocol using an integration approach for hybrid networks," in Proc. IEEE Int. Conf. Green Computing Communication and Electronical Enineering (ICGCCEE), (Coimbatore, Indian), pp. 1-6, 2014. 
[30] V. Arora and C. R. Krishna, "Performance evaluation of routing protocols for MANETs under different traffic conditions," in Proc. IEEE Int. Conf. Computering Engineering and Technology (ICCET), (Chengdu, China), pp. 79-84, 2010.

[31] Y. Singh, A. Kumar, P. Rani, and S. K. Kaushik, "Impact of CBR traffic on routing protocols in MANETs," in Proc. IEEE Int. Conf. Computer Modelling and Simulation, (Cambridge, UK), pp. 474-478, 2014.

[32] F. D. Rango, M. Fotino, and S. Marano, "EE-OLSR: energy efficient OLSR routing protocol for mobile ad-hoc networks," in Proc. Int. Conf. Military Communications (MILCOM), (San Diego, CA), pp. 1-7, 2008.

[33] A. Ouacha, N. Lakki, J. E. Abbadi, A. Habbani, and M. E. Koubti, "OLSR protocol enhancement through mobility integration," in Proc. IEEE Int. Conf. Networking, Sensing, and Control, (ICNSC), (Evry, France), pp. 17-22, 2013.

[34] C. E. Perkins, E. M. Royer, S. R. Das, and M. K. Marina, "Performance comparison of two on-demand routing protocols for ad hoc networks," IEEE Personal Commun. Mag., vol. 8, no. 1, pp. 16-28, 2001.

[35] R. Bhaskar, J. Herranz, and F. Laguillaumie, "Efficient authentication for reactive routing protocols," in Proc. IEEE Int. Conf. Advance Information Networking and Applications (AINA), (Vienna, German), pp. 57-61, 2006.

[36] Istikmal, "Analysis and evaluation optimization dynamic source routing (DSR) protocol in mobile ad hoc network based on ant algorithm," in Proc. IEEE Int. Conf. Information and Communication Technology (ICoICT), (Bandung, Indonesia), pp. 400-404, 2013.

[37] Q. Liu, H. Wang, J. Kuang, Z. Wang, and Z. Bi, "WSNp-1: M-TORA: a TROAbased multi-path routing algorithm for mobile ad hoc networks," in Proc. IEEE Global Tele. Conf. (GLOBOCOM), (San Francisco, CA), pp. 1-5, 2006.

[38] Y. Zhang and T. Gulliver, "Quality of service for ad hoc on-demand distance vector routing," in Proc. IEEE Int. Conf. Wireless And Mobile Computing, Networking And Communications (WiMob), (Montreal, Canada), pp. 192-196, 2005.

[39] Z. Cao and G. Lu, "S-AODV: Sink routing table over AODV routing protocol for 6lowpan," in Proc. IEEE Int. Conf. Networks Security Wireless Communications and Trusted Computing (NSWCTC), (Wuhan, Hubei), pp. 340-343, 2010. 
[40] X. Peng, Y. Wu, Z. Xu, and X. Lin, "Aodv-mr: Aodv with multi-RREP for vanets," in Proc. IEEE Int Conf. Advanced Info.Comm. Tech. (ICAIT), (Fuzhou, China), pp. 172-176, 2014.

[41] K. Jalil, Z. Ahmad, and J. Manan, "Securing routing table update in AODV routing protocol," in Proc. IEEE Int. Conf. Open System (ICOS), (Langkawi, Malaysia), pp. 25-28, 2011.

[42] L. E. Moore and M. B. O’Neal, "A semantic interpreter for a transportable command language interface," in Proc. IEEE Symposium on Applied Computing, (Fayetteville, AR), pp. 202-208, 1990.

[43] "Software defined networking." Website. https://en.wikipedia.org/wiki/Software defined networking.

[44] "SDN and openflow for increased flexibility, improved performance and simplified operations." Website, Oct. $2013 . \quad$ https://www.ibm.com/developerworks/community/blogs/ibmsyssw/entry/sdn openflow for increased flexibility improved performance and simplified operations?lang $=$ zh.

[45] F. Hu, Q. Hao, and K. Bao, "A survey on software-defined network and openflow: From concept to implementation," IEEE Commun. Surveys $\&$ Tutorials, vol. 16, no. 4, pp. 2181-2206, 2014.

[46] "Software-defined networking: the new norm for networks." Website, Apr. 2012. https://www.opennetworking.org/images/stories/downloads/sdn resources/white-papers/wp-sdn-newnorm.pdf.

[47] S. Mehdi, J. Khalid, and S. Khayam, "Revisiting traffic anomaly detection using software defined networking," in Proc. 14th Internaional Symposium on Research in Attacks, Intrusions and Defenses (RAID), (Menlo Park, CA), pp. 161-180, 2011.

[48] G. Ge, Z. Zhen, Y. Wu, and E. Yu, "a heuristic storage space optimization algorithm for flow table of openflow." Website, Jan. 2014. http://onlinelibrary.wiley.com/doi/10.1002/cpe.3206/full.

[49] S. Wallace and C. Small, "Openflow 90 minutes." Website, Oct. 2012. https://www.nanog.org/meetings/nanog57/presentations/OpenFlow.24.pdf. 
[50] "Openflow switch specification." Website, Dec. 2008. http://archive.openflow.org/documents/openflow-spec-v0.8.9.pdf.

[51] B. Xu, S. Hischke, and B. Walke, "The role of ad hoc networking in future wireless communications," in Proc. IEEE Int. Conf. Communication Technology (ICCT), (Beijing, China), pp. 561-566, 2008.

[52] V. Janhavi and B. N. Yuvaraju, "A unified method to solve the energy depletion and congestion problem using mobile relay in MANETs," in Proc. IEEE Int. Conf. Information Technology and Artificial Intelligence (ITAIC), (Chongqing, China), pp. 330-335, 2014.

[53] Z. Wang, J. Lu, and J. Tang, "Neighboring monitor mechanism to solve unidirectional link problem in MANETs," in Proc. IEEE Int. Conf. Wireless and Mobile Communication (ICWMC), (Guadeloupe, France), p. 72, 2007.

[54] M. S. A. Mazrouei and S. Narayanaswami, "Mobile ad hoc networks: a simulation based security evaluation and intrusion prevention," in Proc. IEEE Int. Conf. Internet Technology and Secured Transactions (ICITST), (Abu Dhabi), pp. 308313, 2011.

[55] C. Gong, S. Wu, and Y. Jing, "ARAN protocol analysis and improvement," in Proc. IEEE Int. Conf. System Science, Engineering Design, and Manufacturing Information, (ICSEM), (Chengdu, China), pp. 347-350, 2012.

[56] W. Galuba, P. Papadimitrators, M. Poturalski, K. Aberer, Z. Despotovic, and W. Kellerer, "Castor: scalable secure routing for ad hoc networks," in Proc. IEEE Int. Conf. Computer Commun. (INFOCOM), (San Diego, CA), pp. 1-9, 2010.

[57] N. Marchang and R. Datta, "Light-weight trust based routing protocol for mobile ad hoc networks," IET Inf. Secur, vol. 6, no. 2, pp. 77-83, 2011.

[58] D. Amutha and R. Punitha, "Preventing gray hole attack using AOTMDV routing protocol in MANETs," Int. J. Engineering and Computer Science, vol. 5, no. 4, pp. 16139-16141, 2016.

[59] S. Mukherjee, M. Chattopadhyay, and S. Chattopadhyay, "A novel encounter based trust evaluation for AODV routing in MANETs," in Proc. IEEE Int. Conf. Applications and Innovations in Mobile Computing (AIMoC), (Kolkata, Indian), pp. 141-145, 2015. 
[60] A. Pushpa, "Trust based secure routing in AODV routing protocol," in Proc. IEEE Int. Conf. Internet Multimedia Services Architecture and Applications (IM$S A A)$, (Bangalore, Indian), pp. 1-6, 2009.

[61] M. Amaresh and G. Usha, "Efficient malicious detection for AODV in mobile ad hoc networks," in Proc. IEEE Int. Conf. Internet Multimedia Services Architecture and Applications (IMSAA), (Chennai, Indian), pp. 1-6, 2009.

[62] L. W. X. Li, Z. Jia and H. Wang, "Trust-based on-demand multipath routing in mobile ad hoc networks," IET Inf. Secur, vol. 4, no. 4, pp. 305-317, 2010.

[63] Y. Sun, W. Yu, Z. Han, and K. R. Liu, "Information theoretic framework of trust modeling and ealuation for ad hoc networks," IEEE J. Selected Areas in Communications, vol. 24, no. 2, pp. 212-232, 2006.

[64] M. Jo and K. Kim, "A research on the regional routing scheme based mobile agent for SDN," in Proc. IEEE Int. Conf. Information Networking (ICOIN), (Kota Kinabalu, Malaysia), pp. 211-213, 2016.

[65] Z. Wei, H. Tang, F. R. Yu, M. Wang, and P. Mason, "Security enhancements for mobile ad hoc networks with trust management using uncertain reasoning," IEEE Trans. Veh. Tech., vol. 63, no. 9, pp. 4647-4658, 2014. 


\section{Appendix A}

\section{Simulation Programs}

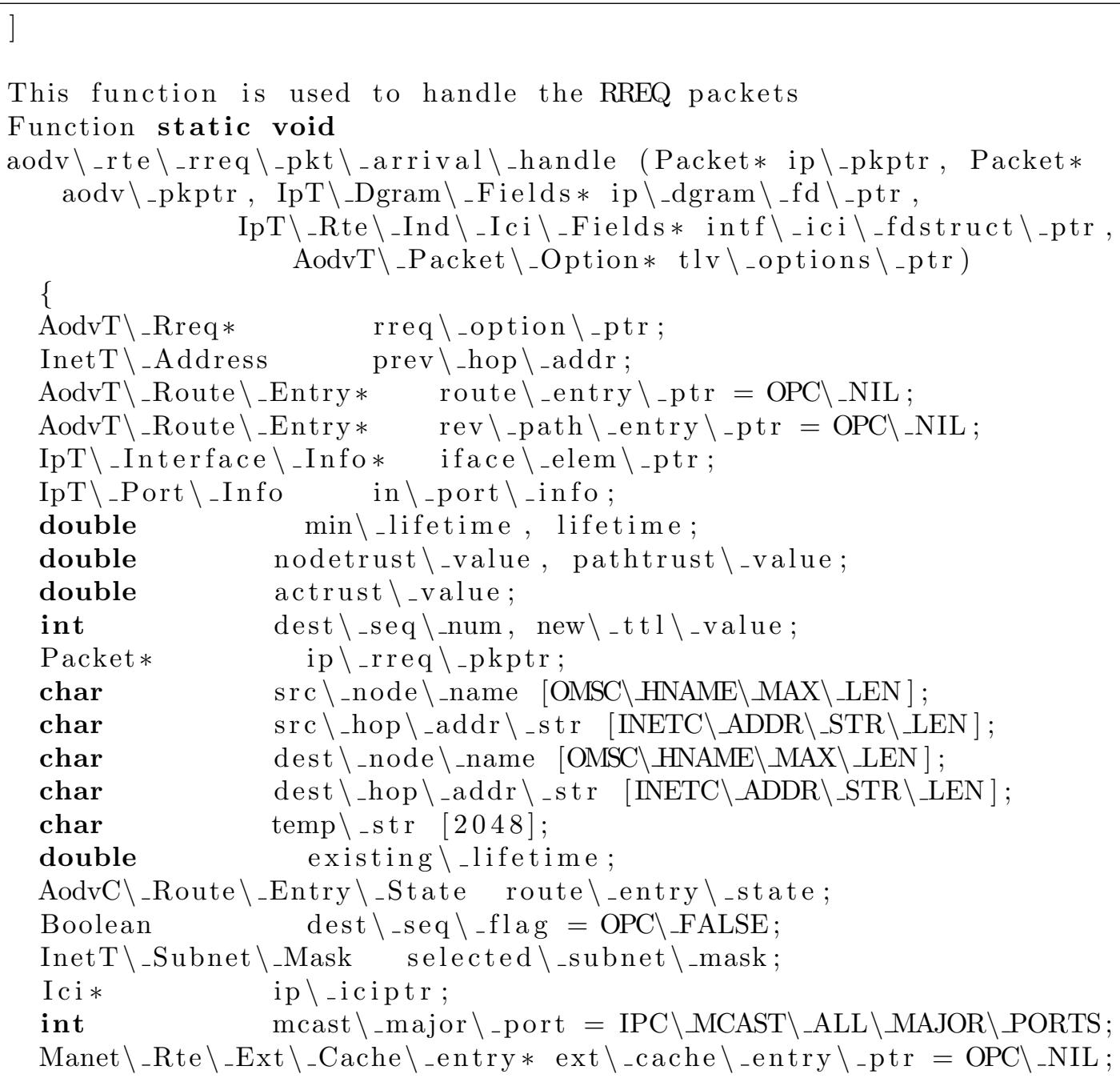


/** Handles the arrival of a route request message **/

FIN (aodv \_rte \_rreq \_pkt\_arrival\_handle $(<\operatorname{args}>))$;

/* A route request packet has arrived at this node */

/* Get the request options from the packet */

rreq \_option $\backslash$-ptr $=(\operatorname{AodvT} \backslash$ _Rreq $*)$ tlv $\backslash$ _options $\backslash$-ptr $\rightarrow>$ value $\backslash$ _ptr;

/* Get the previous hop from which this packet arrived */

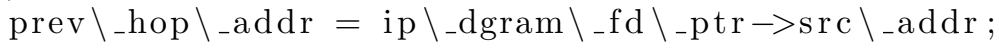

/* The source address needs to be set at the first hop */

/* for the route request as when sending out the RREQ*/

* from the actual source node, the output interface */

/* address is not known as it is broadcast. */

if ( rreq \_option $\backslash_{-}$ptr $\rightarrow$ hop $\backslash_{-}$count $=0$ )

rreq \_option \_ptr->src \_addr = inet \_address \_copy ( prev $\searrow_{-}$hop $\backslash$ _addr);

if ( op $\backslash_{-}$prg $\backslash_{-}$odb $\backslash_{-}$ltrace $\backslash_{\text {_active }}$ ( "trace $\backslash_{-}$rreq") $=$OPC $\backslash_{-}$TRUE $)||$ LTRACE $\backslash$ ACTIVE)

\{

inet $\backslash_{\text {_address } \backslash \text { _print }}$ ( src $\backslash_{\text {_hop }} \backslash_{\text {_addr }} \backslash_{\text {_str }}$,

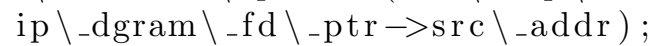

inet \_address \_to \_hname (ip \_dgram \_fd \_ptr $\rightarrow$ src $\backslash$ _addr , src \_node $\backslash_{-}$name) ;

inet $\backslash$ _address $\backslash$ _print (dest $\backslash_{\text {_hop }} \backslash_{\text {_addr }} \backslash_{\text {_str }}$, rreq \_option \_ptr->dest \_addr);

inet \_address \_to\_hname (rreq\_option \_ptr $\rightarrow$ dest \_addr, dest \_node \_name);

sprintf (temp \-str, "from node \%s (\%s) destined to node \%s (\%s) with request ID $(\% \mathrm{~d}) "$,

src \_hop \_addr \_str, src \_node \_name, dest \_hop \_addr \_str, dest \_node \_name, rreq \_option \_ptr->rreq\_id );

op \_prg \_odb\_print\_major (pid \_string, "Received a Route Request", temp \_str , OPC \_NIL );

\}

/* Update the connectivity table */

aodv \_rte \_neighbor \_connectivity \_table \_update (prev \_hop \_addr, $\mathrm{OPC} \backslash$ FALSE);

/* Update the route to the previous hop of the */

/* route request message. */

aodv $\backslash_{-} r t e \backslash_{-}$route $\backslash_{-}$table $\backslash_{-}$entry $\backslash_{-}$update (ip $\backslash_{-}$dgram $\backslash_{-} f d \backslash_{-}$ptr , intf \_ici \_fdstruct \_ptr, tlv \_options \_ptr);

* If the source address of the route request */

/* belongs to this node, then discard the IP */

/* datagram as it has received its own packet */ 


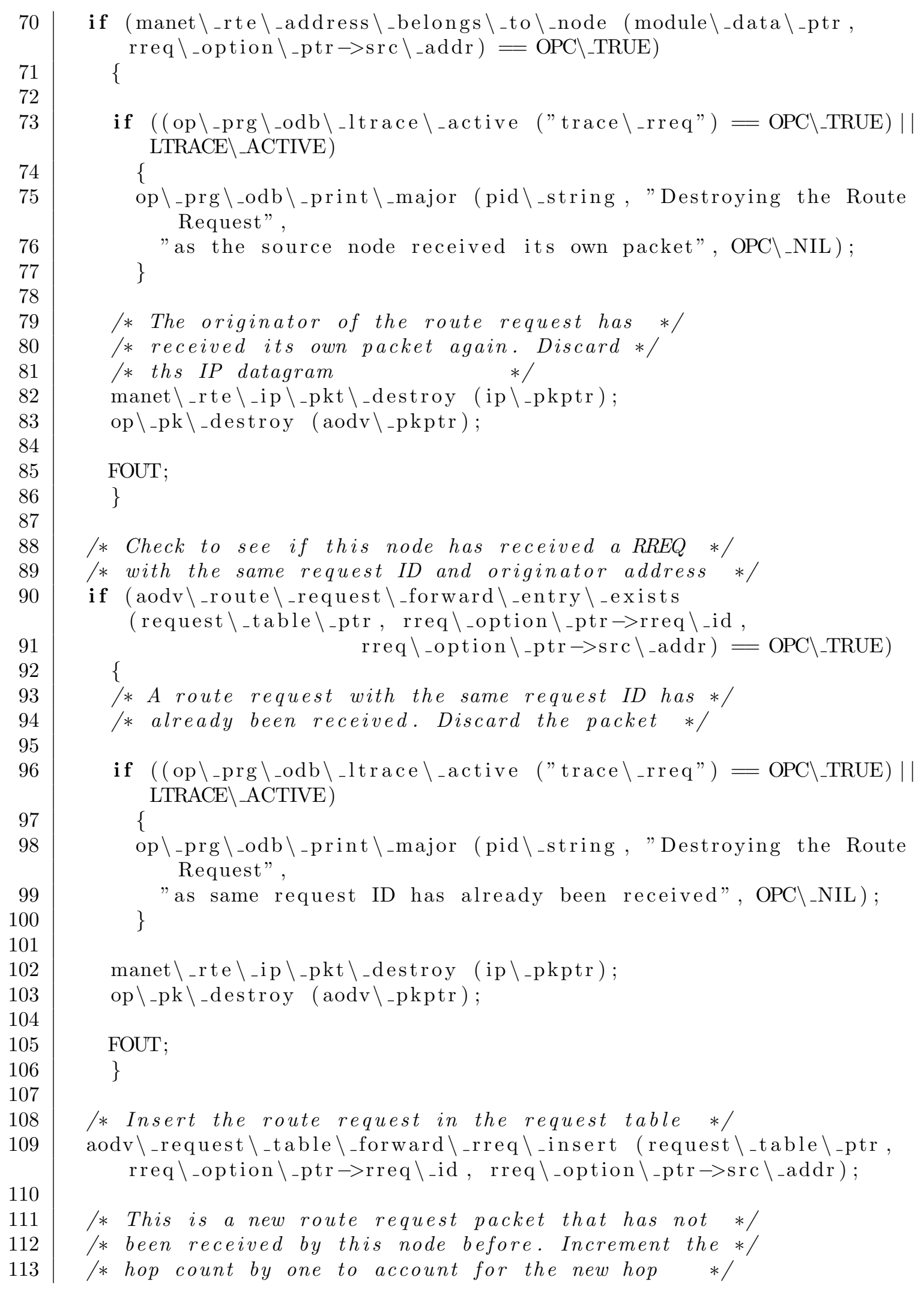




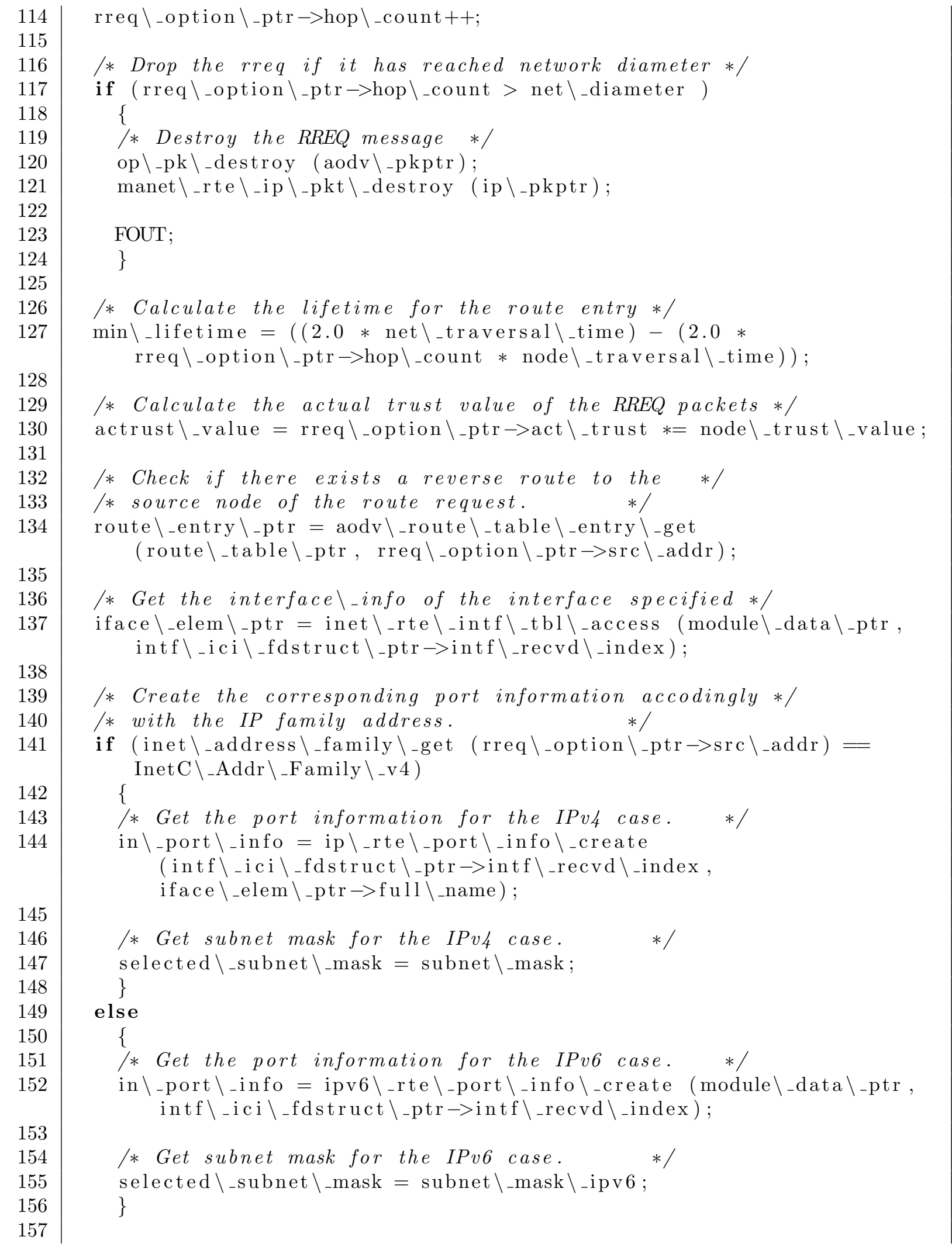




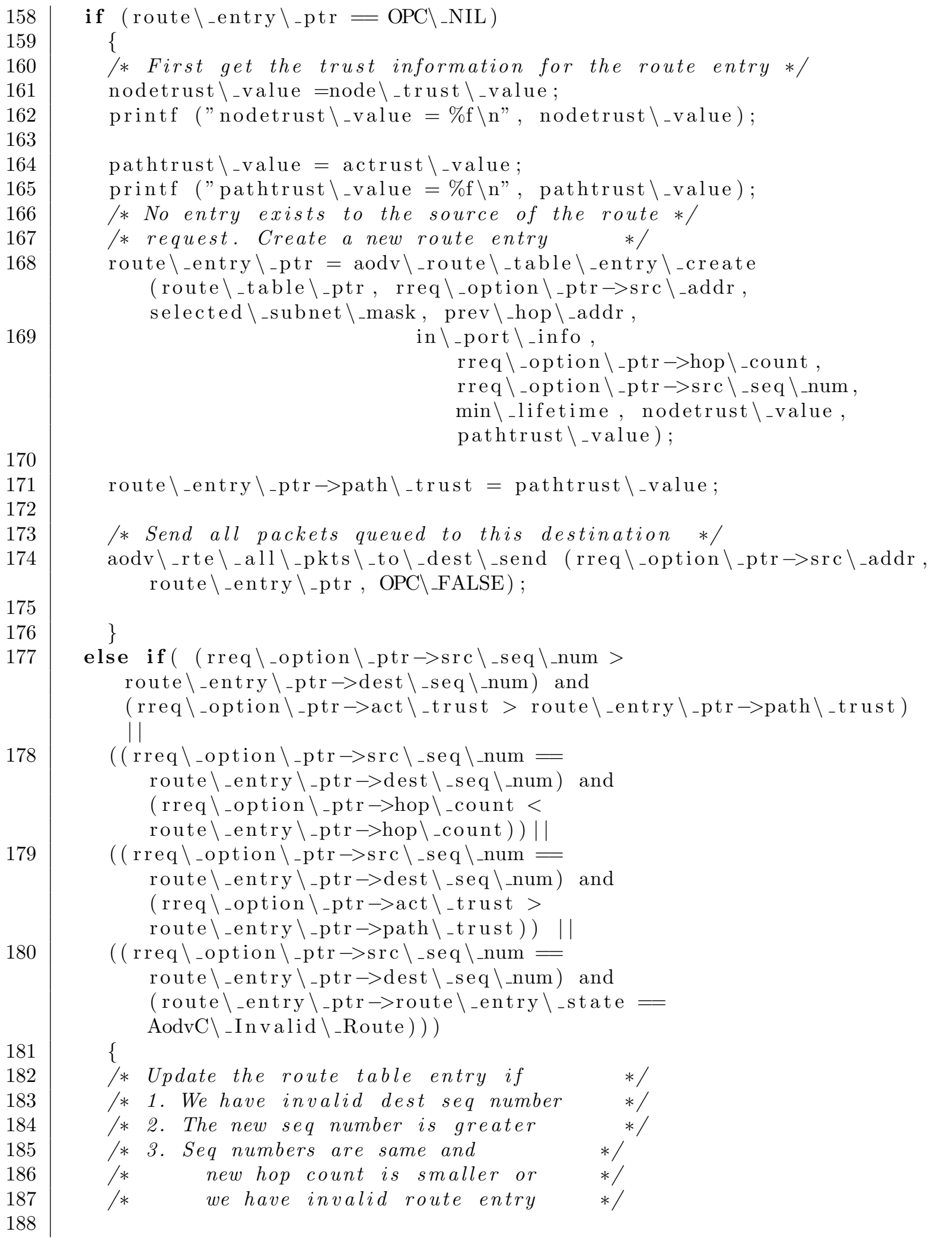


if ( op \_prg \_odb \_ltrace \_active ("trace \_rreq") $==$ OPC $\backslash$ _TRUE $)||$ LTRACE $\backslash$ ACTIVE)

\{

printf("Route entry already exists: Old dest \_seq\_num: \%d, Old Hop Cnt: \%d\n",

route \_entry $\backslash_{-}$ptr $\rightarrow$ dest $\backslash_{-}$seq $\backslash_{\text {_num }}$, route $\backslash_{-}$entry $\backslash_{-}$ptr $\rightarrow$ hop $\backslash_{-}$count );

printf("Route entry already exists: new dest \_seq\_num: \%d, new Hop Cnt: \%d $\backslash \mathrm{n} "$,

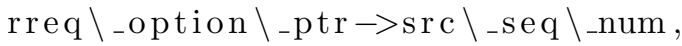
rreq \_option $\backslash_{-}$ptr $\rightarrow$ hop $\backslash_{-}$count );

printf("Route entry already exists: act \_trust: \%f, path \_trust: $\% \mathrm{f} \backslash \mathrm{n} "$,

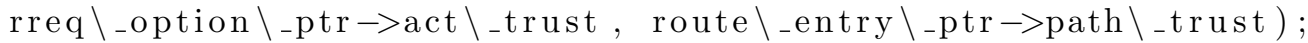
op \_prg \_odb\_print \_major (pid \_string, "Updating route table with new RREQ", OPC $\backslash$-NIL); \}

/* Update the sequence number */ route \_entry _ptr $_{-}>$dest $\backslash$ _seq $\backslash$ _num $=$ rreq \_option \_ptr->src \_seq \_num;

* Update the path trust value */

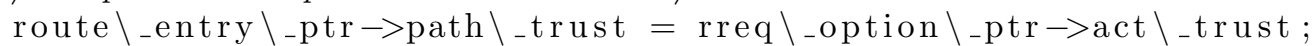

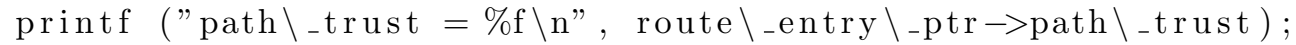

/* Do this before adding info to IP Cmn table in case route was invalid */

route $\backslash_{-}$entry $\backslash_{-}$ptr $\rightarrow$ hop $\backslash_{-}$count $=$rreq $\backslash_{-}$option $\backslash_{-}$ptr $\rightarrow$ phop $\backslash_{-}$count;

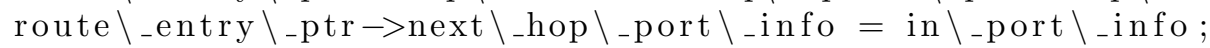

if (! inet \_address $\backslash_{-}$equal (route $\backslash_{\text {_entry }} \backslash_{\text {_ptr }} \rightarrow$ - next $\backslash_{-}$hop $\backslash_{\text {_addr }}$, prev \_hop \_addr)) \{ /* Update the route table entry */ aodv \_route \_table \_entry \_next \_hop \_update (route $\backslash_{-}$table $\backslash_{-}$ptr, route \_entry $\backslash_{-}$ptr, inet $\backslash_{\text {_address }} \backslash_{\text {_copy }}$ ( prev $\backslash_{-}$hop $\backslash_{\text {_addr }}$ ), \} rreq $\backslash_{-}$option $\backslash_{-}$ptr $\rightarrow$ hop $\backslash_{-}$count, in $\backslash_{-}$port $\backslash_{-}$info);

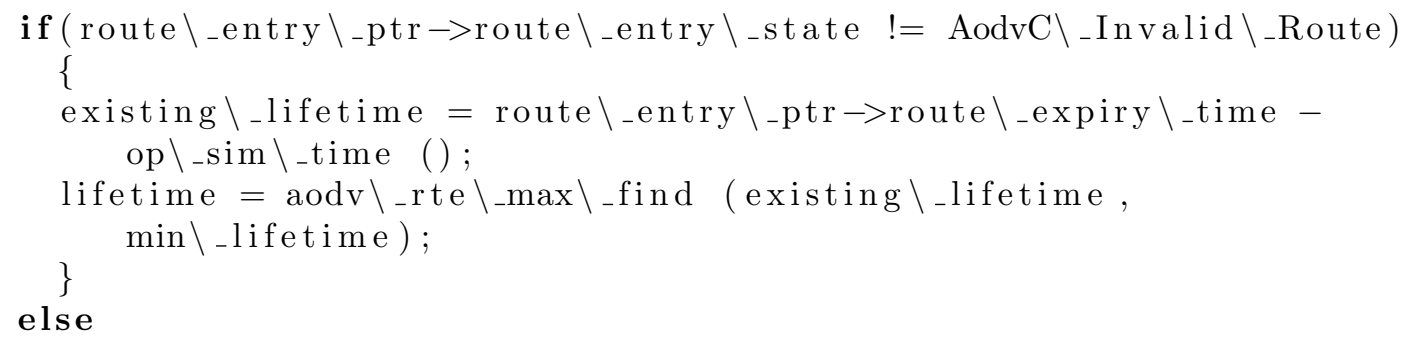




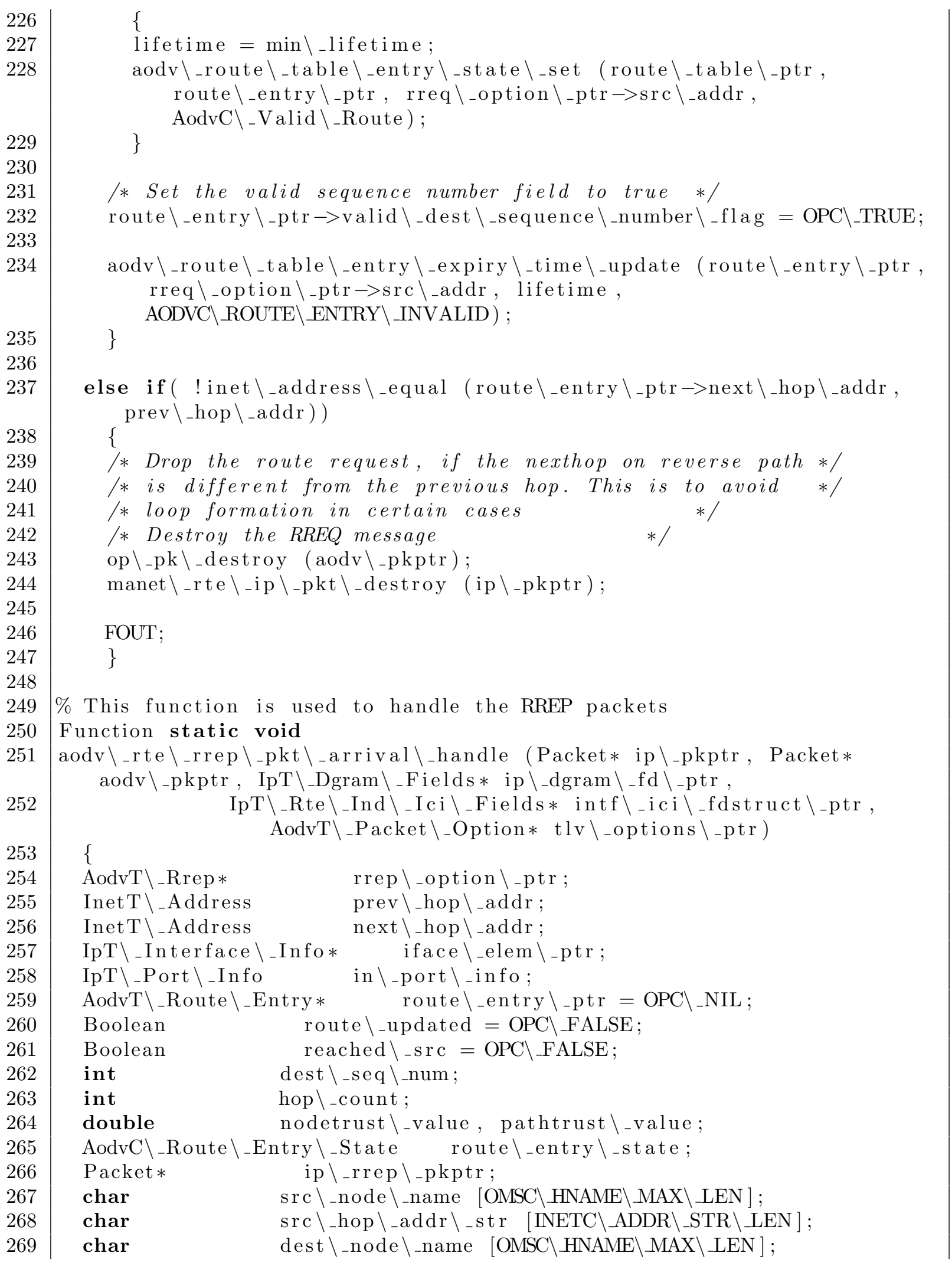




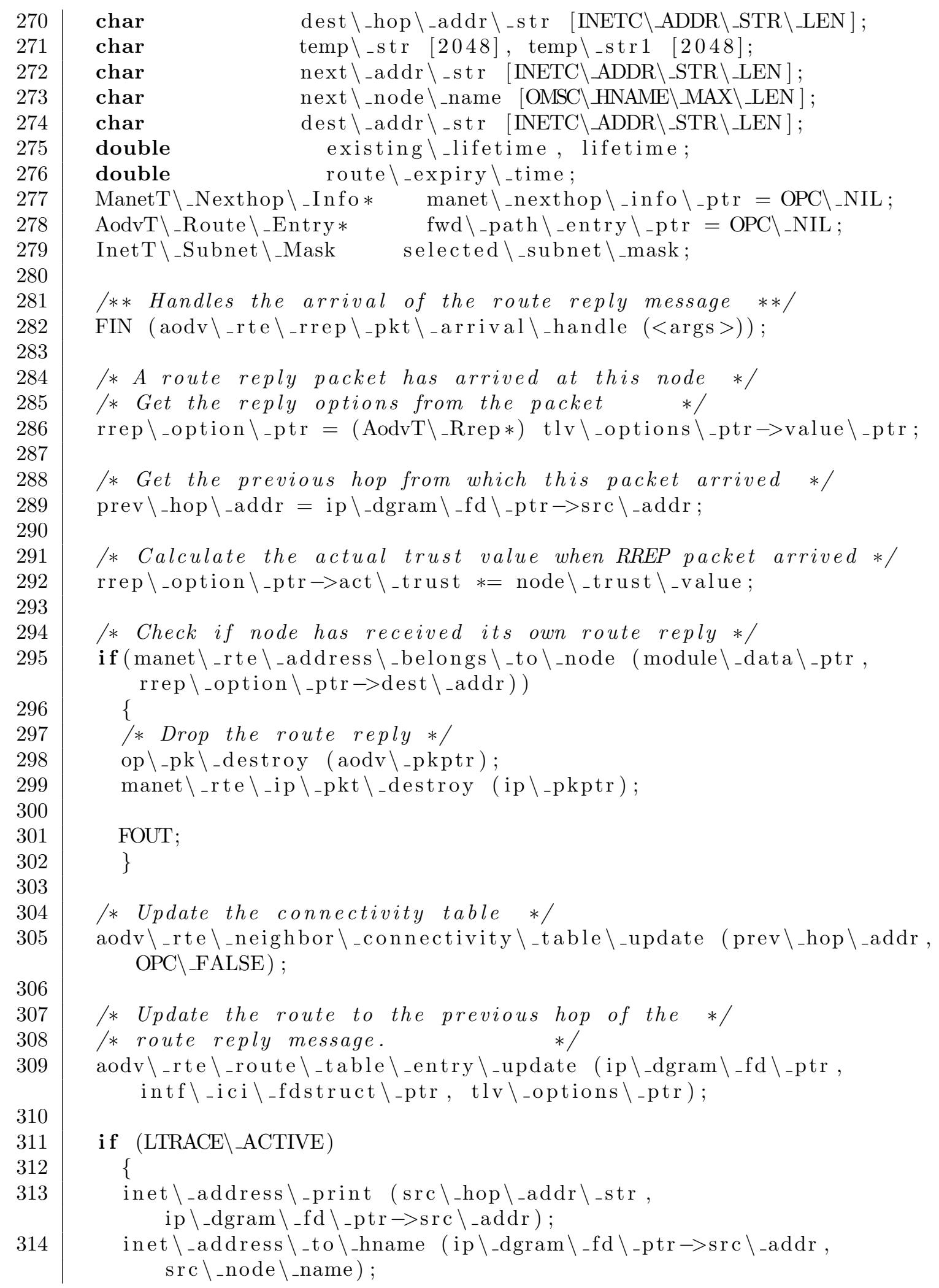


inet $\backslash$ _address $\backslash$ _print (dest $\backslash$ _hop $\backslash$ _addr $\backslash$ _str , rrep \_option \_ptr->dest \_addr); inet \_address \_to\_hname (rrep\_option \_ptr $\rightarrow$ dest $\backslash$ _addr, dest \_node \_name);

sprintf (temp $\backslash$-str, "from node \%s (\%s). Originator of this RREP: \%s $(\% \mathrm{~s}) "$,

src \_hop \_addr \_str, src \_node \_name, dest \_hop \_addr \_str, dest \_node \_name);

op \_prg \_odb\_print \_major (pid \_string, "Received a route reply packet", temp $\backslash_{-}$str , OPC $\backslash_{-}$NIL );

/* Increment the hop count by one to account */

/* for the new hop through the intermediate node */

rrep \_option $\backslash_{\text {_ptr }} \rightarrow$ hop $\backslash$ _count ++ ;

/* Drop the rreq if it has reached network diameter */

if (rrep \_option $\backslash_{-}$ptr $\rightarrow$ hop $\backslash_{-}$count $>$net $\backslash_{-}$diameter )

\{

* Destroy the RREQ message */

op \_pk _destroy (aodv \_pkptr);

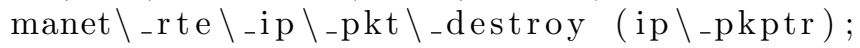

FOUT;

\}

/* Get the interface \_info of the interface specified */

iface $\backslash_{-}$elem $\backslash_{-}$ptr $=$inet $\backslash_{-}$rte $\backslash_{-}$intf $\backslash_{-}$tbl $\backslash_{-}$access (module $\backslash_{-}$data $\backslash_{-} p t r$,

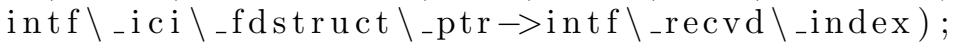

/* Check for the IP address family. */

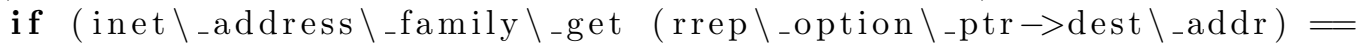
Inet $C \backslash_{-}$Addr $\backslash_{-}$Family $\backslash_{-}$- 4$)$ \{ /* Get the port information for the IPv4 case. */ in $\backslash$ _port $\backslash$ _info $=$ ip $\backslash$ _rte $\backslash$ _port $\backslash$ _info $\backslash$ _create (intf $\backslash_{-}$ici $\backslash_{-}$fdstruct $\backslash_{-}$ptr $\rightarrow$ intf $\backslash_{-}$recvd $\backslash_{-}$index , if ace $\backslash_{-}$elem $\backslash_{-}$ptr $\rightarrow$ full $\backslash_{-}$name);

/* Get subnet mask for the IPv4 case. */ selected \_subnet \_mask = subnet \_mask;

\}

else

\{

* Get the port information for the IPv6 case. */

in \_port \_info = ipv6 \_rte \_port\_info \_create (module \_data \_ptr, intf $\backslash_{-}$ici $\backslash_{-}$fdstruct $\backslash_{-}$ptr $\rightarrow$ intf $\backslash_{-}$recvd $\left.\backslash_{\text {_index }}\right)$;

/* Get subnet mask for the IPv6 case.

selected \_subnet \_mask = subnet \_mask \_ipv6; \} 
/* Check if this is the final destination of the RREP message */ reached $\backslash_{\text {_src }}=$ manet $\backslash$ _rte $\backslash$ _address $\backslash$ _belongs $\backslash$ _to $\backslash$ _node

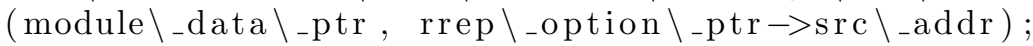

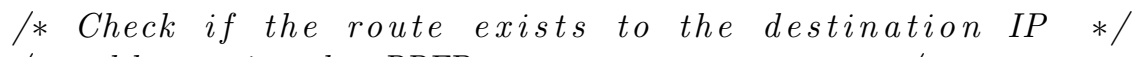


464

465

466

467

468

469

470 inet \_address \_copy (prev \_hop \_addr), rrep \_option \_ptr $\rightarrow$ hop $\backslash_{-}$count in $\backslash$ - port $\backslash$ _info);

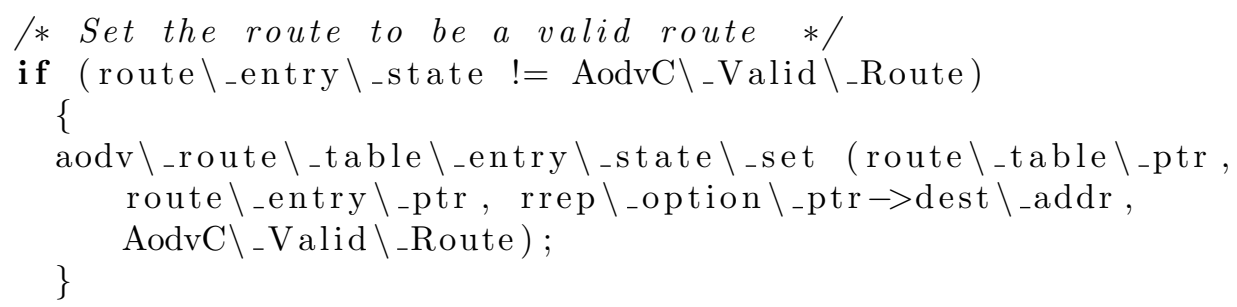




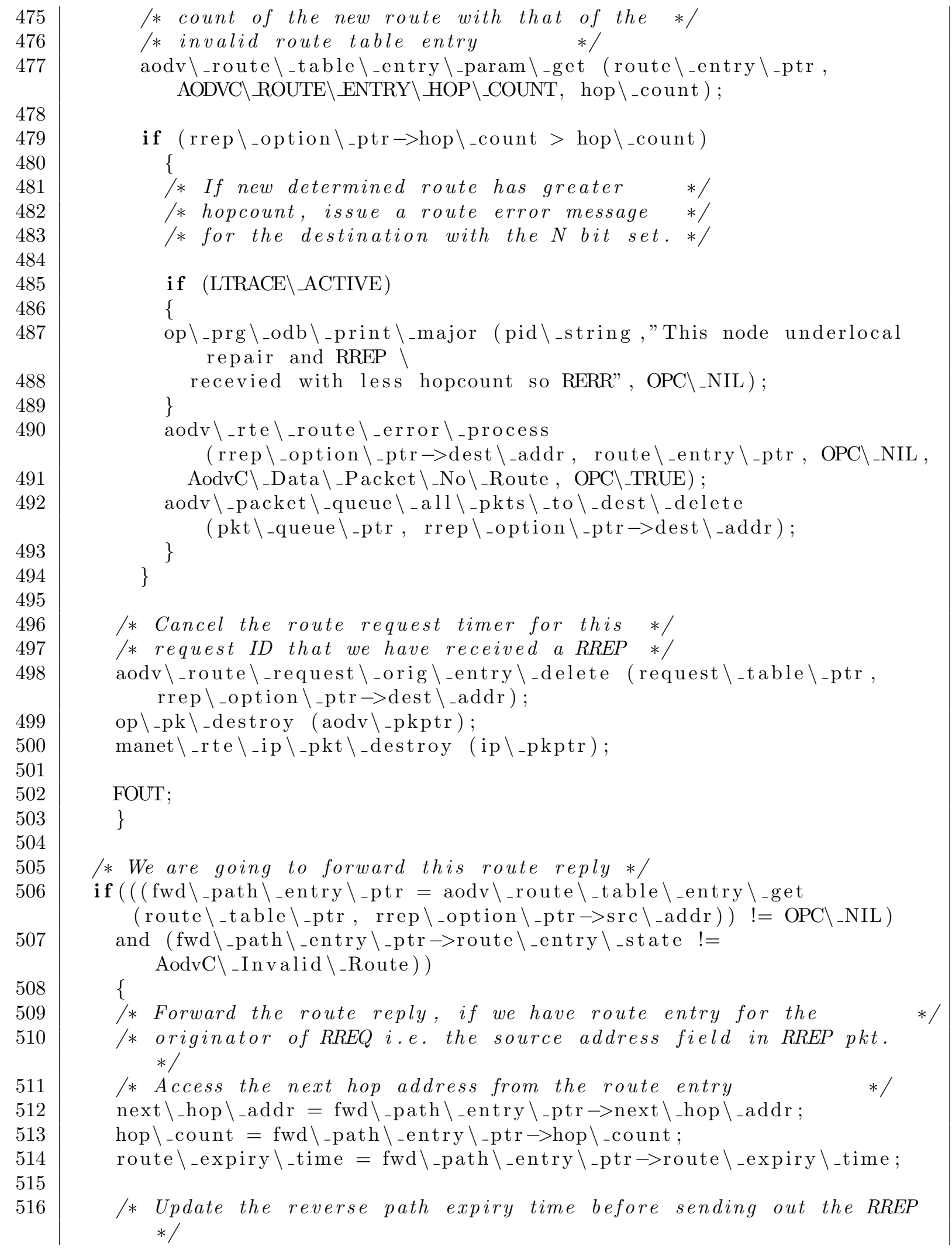




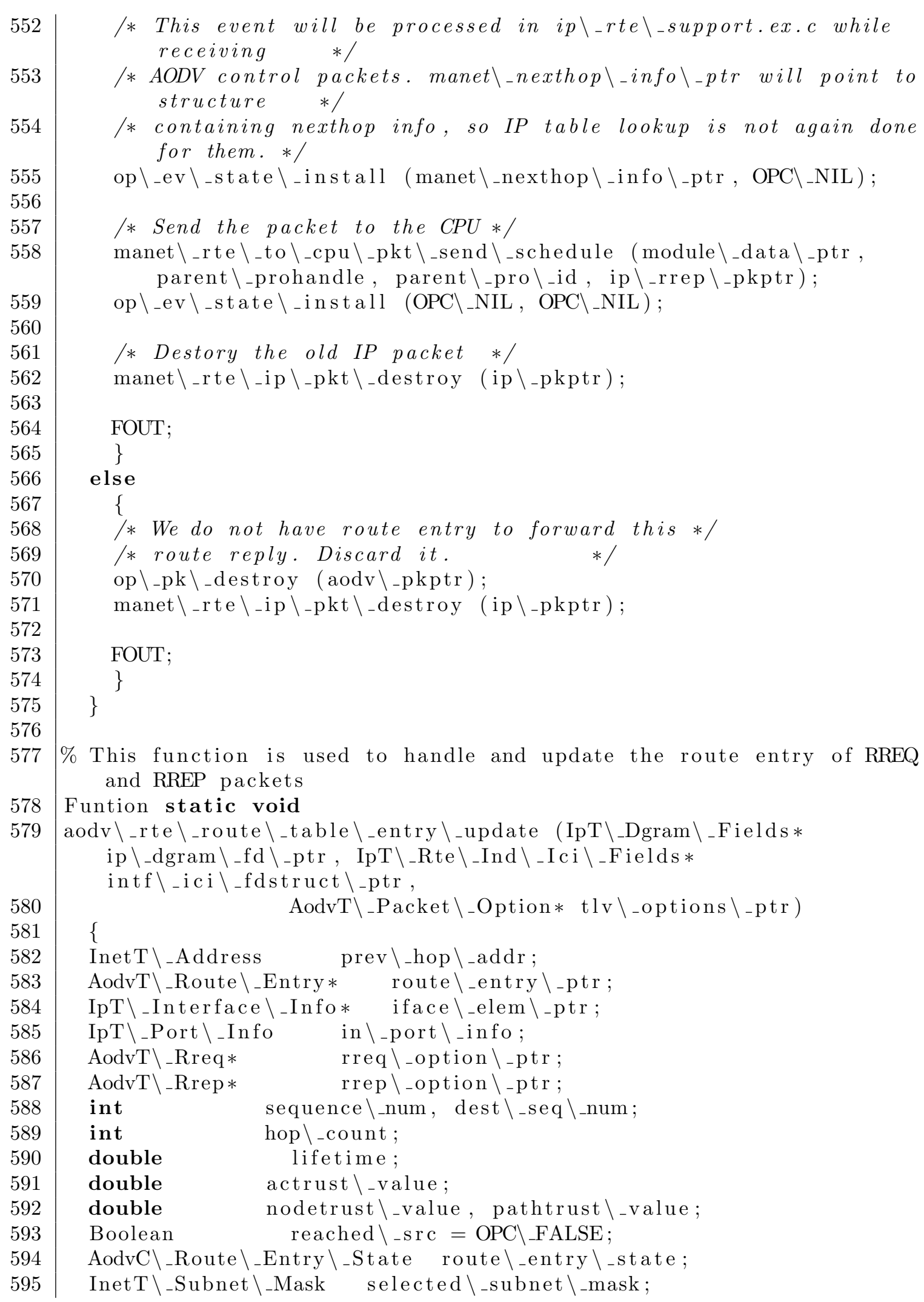

/* This event will be processed in ip \-rte\_support.ex.c while receiving */

/* AODV control packets. manet \_nexthop \_info\_ptr will point to structure */

* containing nexthop info, so IP table lookup is not again done for them. */ 


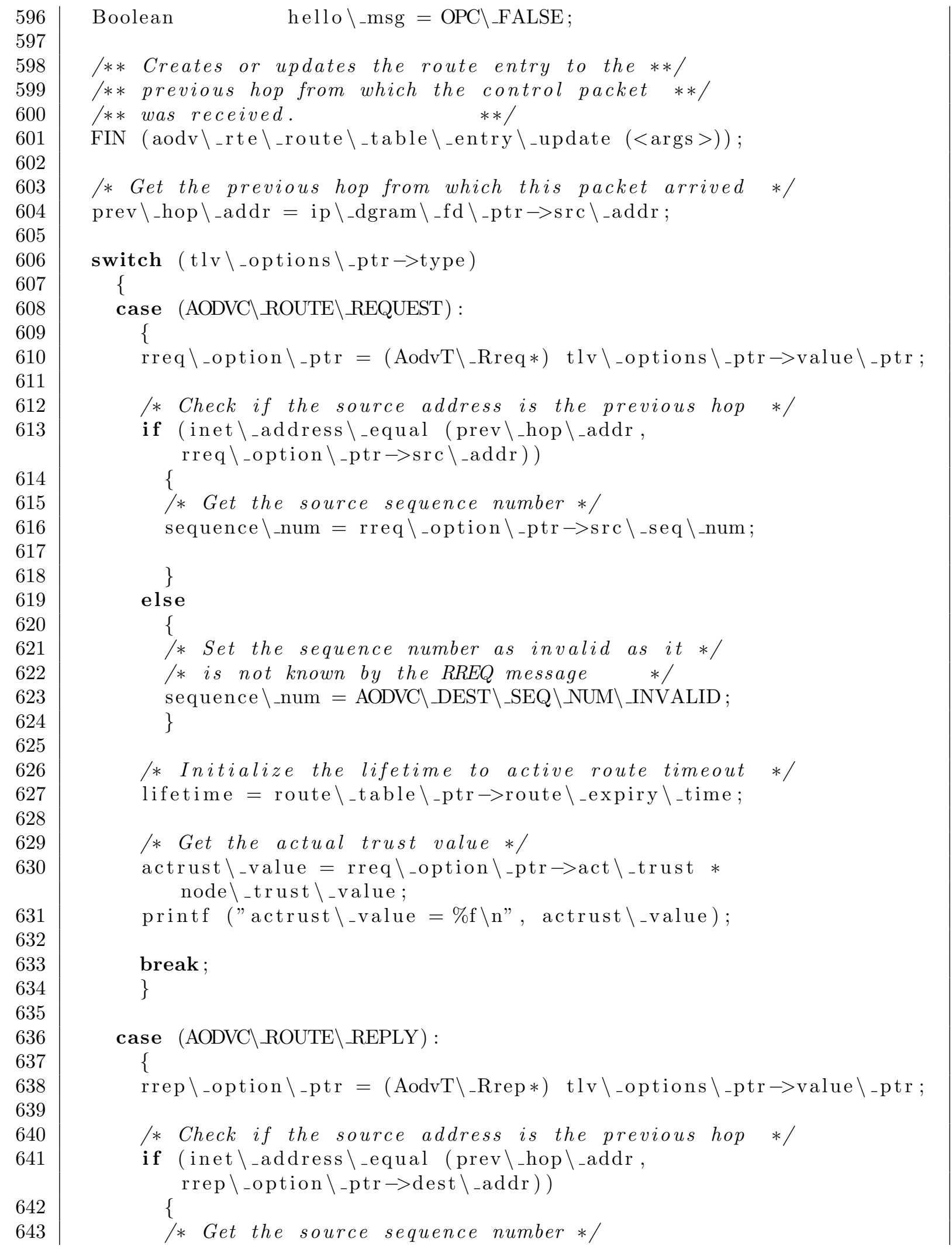




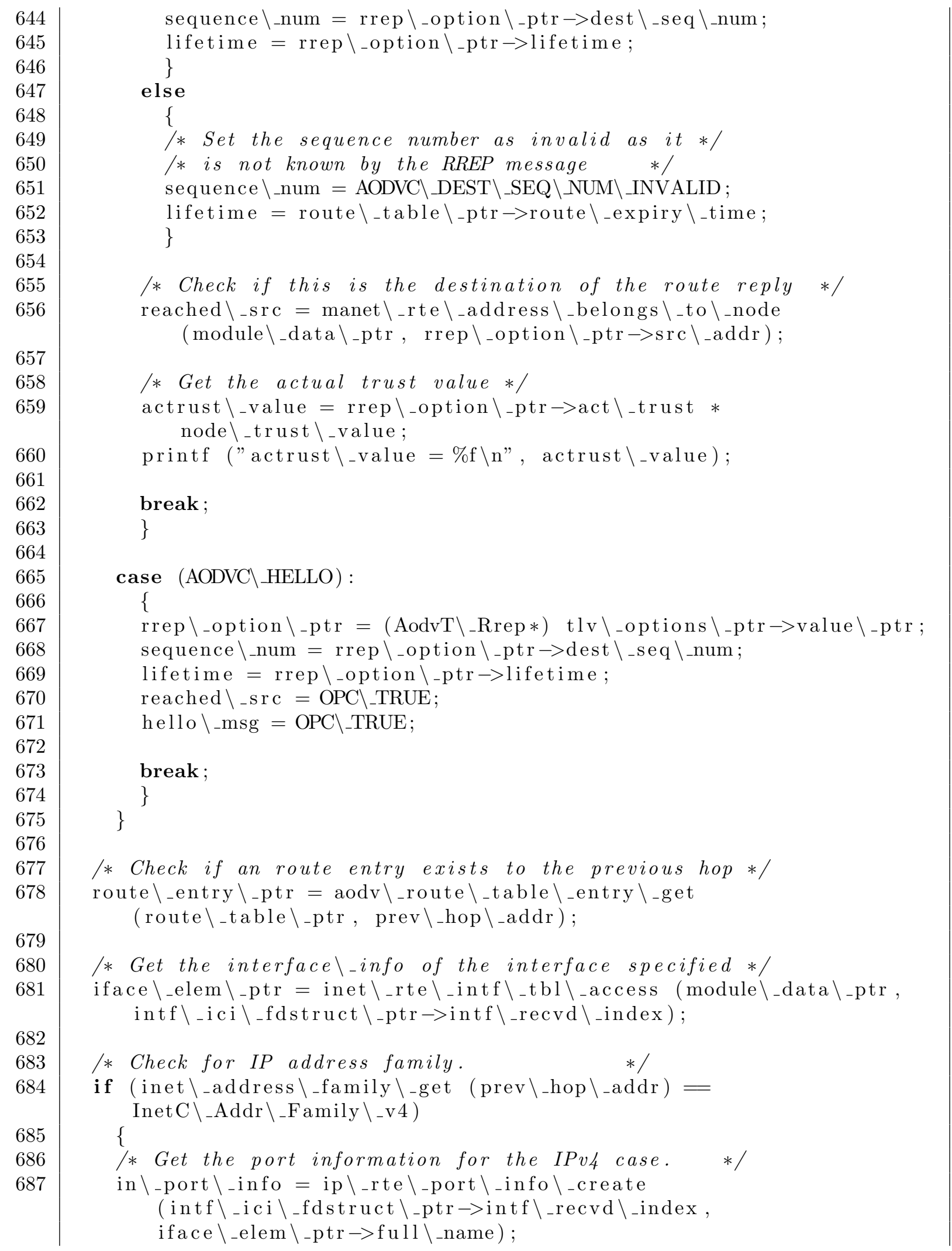




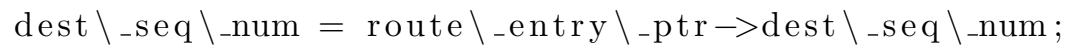

hop $\backslash_{-}$count $=$route $\backslash_{-}$entry $\backslash_{-}$ptr $\rightarrow$ hop $\backslash_{-}$count;

pathtrust $\backslash$-value $=$ route $\backslash$ _entry $\backslash$ _ptr $\rightarrow$ path $\backslash$ _trust;

route $\backslash_{-}$entry $\backslash_{-}$state $=$route $\backslash_{-}$entry $\backslash_{-}$ptr->route $\backslash_{-}$entry $\backslash_{-}$state;

/* Update the route entry if the received packet has higher dest seq no */

/* or same seq no with better hop count or my route entry is invalid */

/* In case of hello message, always update the route entry

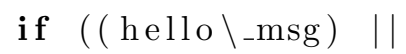

( sequence \_num > dest \_seq \_num) and (actrust \_value > pathtrust $\backslash$-value $)||$

( ( sequence $\backslash$ num $=$ dest $\backslash_{-}$seq $\backslash$ num) and (route $\backslash_{-}$entry $\backslash_{-}$state $=$ AodvC $\backslash$ _Invalid \_Route ) ) ||

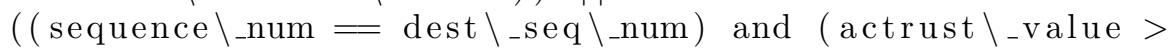
pathtrust $\backslash$-value $))||$

$\left(\left(\right.\right.$ sequence $\backslash$ num $=$ dest $\backslash_{-}$seq $\backslash$ nnum $)$ and $\left(\right.$ hop $\backslash_{-}$count $\left.\left.\left.<2\right)\right)\right)$ \{

/* Do this before adding info to IP Cmn table in case route was invalid */

route \_entry $\backslash_{\text {_ptr }} \rightarrow$ dest $\backslash_{\text {_seq }} \backslash$ _num $=$ sequence $\backslash$ _num;

pathtrust $\backslash$-value $=$ actrust $\backslash$-value;

printf ( "pathtrust \_value \%f \n", pathtrust \_value);

route $\backslash_{-}$entry $\backslash_{-}$ptr $\rightarrow$ hop $\backslash_{-}$count $=1$;

route \_entry _ptr $_{-}>$next $\backslash_{\text {_hop }} \backslash_{\text {_port }} \backslash_{\text {_info }}=$ in $\backslash_{\text {_port }} \backslash_{\text {_info; }}$;

if (! inet \_address \_equal (route \_entry \_ptr $\rightarrow$-next \_hop \_addr, prev \_hop \_addr)) \{

/* Update the next hop and the hop count to the new value*/ aodv \_route \_table \_entry \_next\_hop \_update

(route $\backslash_{-}$table $\searrow_{-}$ptr, route $\backslash_{-}$entry $\backslash_{-}$ptr

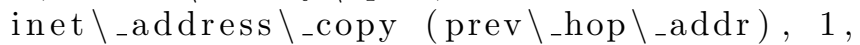
in $\backslash_{\text {_port }} \backslash_{-}$info);

\}

if $\left(\left(\right.\right.$ route $\backslash_{-}$entry $\backslash_{-}$state $=$AodvC $\backslash$ _Invalid $\backslash_{-}$Route $\left.)\right)$

\{

/* If Route was invalid, make it Valid, and add this to the IP Common table */

aodv \_route $\backslash_{-}$table $\backslash_{-}$entry $\backslash_{-}$state $\backslash_{-}$set (route $\backslash_{-}$table $\backslash_{-} p t r$,

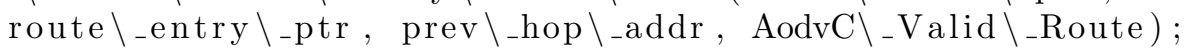
\}

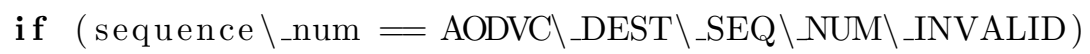




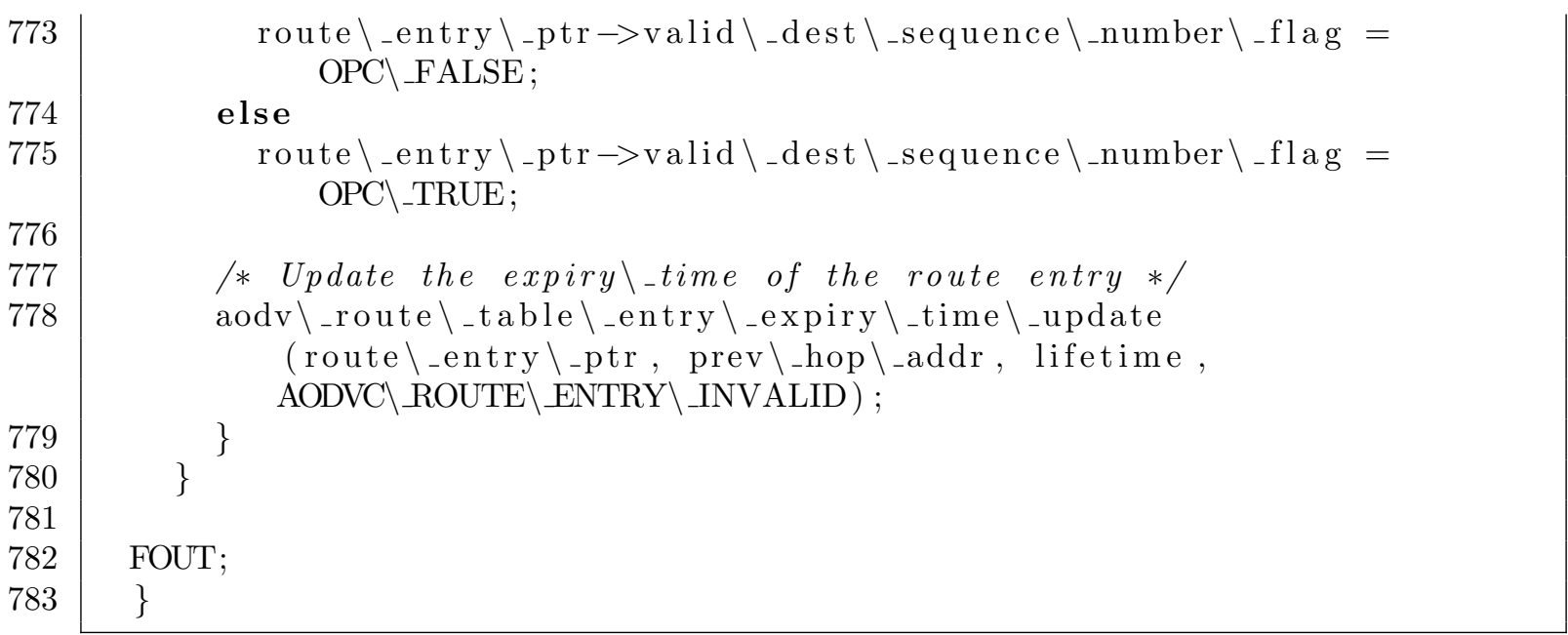

